\title{
Small-Molecule Diversity Using a Skeletal Transformation Strategy
}

Nilesh Kumar, Masatoshi Kiuchi, John A. Tallarico, Stuart L. Schreiber*

\section{Supporting information}

General. All commercially available materials were used without further purification. All reaction solvents except DMF were dispensed from a solvent purification system wherein solvents are passed through packed activated alumina column. DMF was Aldrich anhydrous grade. Solvents for other uses (beads washing, chromatography) were commercially available HPLC grade purchased from Fisher. Solution phase reactions were monitored by thin layer chromatography using Merck silica gel $60 \mathrm{~F}_{254}$ plate $(0.25 \mathrm{~mm}$ thickness $)$. Compounds were visualized by UV $(254 \mathrm{~nm})$ and phosphomolybdic acid. Flash column chromatography was performed using Merck silica gel 60 (230-400 mesh). Infrared spectra were recorded on a Bruker Vector22 spectrometer as a thin film on a $\mathrm{NaCl}$ plate. The absorption maxima (nmax) are reported in wavenumbers $\left(\mathrm{cm}^{-1}\right)$. All NMR spectra were recorded on a Varian Inova AS500 spectrometer. Chemical shifts are expressed in ppm relative to residual solvent signals $\left(\mathrm{CDCl}_{3} 7.27\right.$ $\mathrm{ppm} / 77.23 \mathrm{ppm}$; DMSO- $\left.d_{6} 2.50 \mathrm{ppm} / 39.51 \mathrm{ppm}\right)$. Peak assignments were supported by DEPT, ${ }^{1} \mathrm{H}-{ }^{1} \mathrm{H}$ COSY, gHMQC and gHMBC spectra. LC-MS was performed on a Waters Alliance 2690 HPLC system with a Waters Symmetry C18 column. Compounds were detected by a Waters 996 photo diode array detector and a Micromass LCZ (ESI) spectrometer. $\mathrm{CH}_{3} \mathrm{CN}$ and $0.1 \%$ formic acid in water were used as solvents, and the ratio was $15 \% \mathrm{CH}_{3} \mathrm{CN} / 85 \%$ water at $0 \mathrm{~min}$ and $100 \% \mathrm{CH}_{3} \mathrm{CN}$ at $5 \mathrm{~min}$ with linear gradient followed by $1 \mathrm{~min}$ of $100 \% \mathrm{CH}_{3} \mathrm{CN}$. Analysis of chemical tags oxidatively cleaved from beads was done by a Hewlett Packard HP6890 GC system.

\section{Experimental procedures.}

1) Washing beads after reaction and cleavage of compound from solid support

Washing protocol. After reaction, the beads were drained, exposed to atmosphere, and subjected to the following washing protocol: DMF (15 $\min \times 2), \mathrm{CH}_{2} \mathrm{Cl}_{2}(15 \min \times 2)$, THF (15 $\left.\min \times 2\right)$, THF/IPA (3:1, $15 \min \times 2), T H F / H_{2} \mathrm{O}(3: 1,15 \min x 2)$, THF/IPA $(3: 1,15 \min \times 2)$, THF (15 $\min x$ $2)$, and $\mathrm{CH}_{2} \mathrm{Cl}_{2}(15 \min \times 2)$.

Cleavage protocol. All reactions on solid phase were analyzed by LC-MS after cleavage of the compounds from solid support. A solution of HF/pyridine (Aldrich) and pyridine in THF (5:5:90, 30 $\mathrm{ml}$ ) was added to a vacuum-dried bead in a scintillation vial. The vial was sealed and agitated for 3 hr. Methoxytrimethylsilane $(30 \mathrm{ml})$ was added to the mixture to quench excess HF. The mixture was agitated $15 \mathrm{~min}$. The solution was removed and the bead was washed with THF (30 $\mathrm{ml} \times 2)$. All solutions were combined and concentrated in vacuo. The residue was dissolved in $\mathrm{MeOH}(100 \mathrm{ml})$ to be analyzed by LC-MS.

\section{2) Synthesis of starting material (2 and $\mathbf{3})$}<smiles>C[C@]12CC[C@@H](O)CC1=CC=C1[C@@H]2CC[C@]2(C)[C@@H]1CC[C@@]21CO1</smiles>

Spiro-17b-oxiranylandrosta-5,7-dien-3b-ol (2). $35.0 \mathrm{~g}$ of commercially available $\mathbf{1}$ was converted to $8.92 \mathrm{~g}$ of 3b-3-hydroxyandrosta-5,7-dien-17-one (SI-1) by a reported method. 2 was synthesized by a slightly modified Corey-Chaykovsky method from SI-1. NaH (60\% suspension in mineral oil, $6.24 \mathrm{~g}, 156$ mmol) was added to DMSO $(106 \mathrm{ml})$ at room temperature. The mixture was heated at $70{ }^{\circ} \mathrm{C}$ for $1 \mathrm{hr}$, diluted with THF $(106 \mathrm{ml})$, then cooled to $0{ }^{\circ} \mathrm{C}$. A suspension of trimethylsulfonium iodide $(31.8 \mathrm{~g}, 56$ mmol) in DMSO $(245 \mathrm{ml})$ was added to the cooled mixture at $0{ }^{\circ} \mathrm{C}$. The mixture was stirred at $0{ }^{\circ} \mathrm{C}$ for 20 min. A solution of SI-1 in THF (314 ml) was added to the mixture at $0{ }^{\circ} \mathrm{C}$, and the mixture was stirred at room temperature for $20 \mathrm{hr}$. The reaction mixture was poured into water $(1.3 \mathrm{~L})$, and extracted with diethyl ether ( $1 \mathrm{~L} \times 1,500 \mathrm{ml} \times 2$ ). Combined organic solution was washed by water (1.5 $\mathrm{L} \times 1)$ and brine (1 L x 3), dried over $\mathrm{MgSO}_{4}$, then concentrated. Purification by column chromatography (Hexane/EtOAc $=6 / 4)$ followed by recrystallization in diethyl ether provided $2(3.10 \mathrm{~g}, 33 \%)$ as a white crystalline solid: $\mathrm{mp} 173{ }^{\circ} \mathrm{C}$; IR (thin film) $3471,2967,2934,764,750 \mathrm{~cm}^{-1} ;{ }^{1} \mathrm{H}$ NMR $\left(500 \mathrm{MHz}, \mathrm{DMSO}-d_{6}\right) \delta 5.51(\mathrm{dd}$, 
$1 \mathrm{H}, J=5.9,2.0 \mathrm{~Hz}, \mathrm{C} 6-\mathrm{H}), 5.42(\mathrm{dt}, 1 \mathrm{H}, J=5.9,2.4 \mathrm{~Hz}, \mathrm{C} 7-\mathrm{H}), 4.67(\mathrm{~d}, 1 \mathrm{H}, J=4.4 \mathrm{~Hz}, \mathrm{C} 3-\mathrm{OH})$, 3.39-3.33 (m, 1H, C3-H), $2.82(\mathrm{~d}, 1 \mathrm{H}, J=5.4 \mathrm{~Hz}, \mathrm{C} 20-\mathrm{H}), 2.59$ (d, 1H, J = 5.4 Hz, C20-H), 2.32 (ddd, $1 \mathrm{H}, J=14.6,4.6,2.4 \mathrm{~Hz}, \mathrm{C} 4-\mathrm{H}), 2.16-2.09$ (m, 2H, C4-H, C14-H), 1.96-1.91 (m, 1H, C9-H), 1.91-1.85 (m, 2H, C16- $\left.\mathrm{H}_{2}\right), 1.84-1.77$ (m, 2H, C1-H, C15-H), 1.74-1.70 (m, 1H, C2-H), 1.61-1.56 (m, 3H, C11- $\mathrm{H}_{2}$, C15-H), 1.35-1.29 (m, 2H, C2-H, C12-H), 1.24-1.18 (m, 1H, C1-H), 1.19-1.15 (m, 1H, C12-H), 0.86 (s, $\left.3 \mathrm{H}, \mathrm{C} 19-\mathrm{H}_{3}\right), 0.72\left(\mathrm{~s}, 3 \mathrm{H}, \mathrm{C} 18-\mathrm{H}_{3}\right) ;{ }^{13} \mathrm{C} \mathrm{NMR}\left(125 \mathrm{MHz}, \mathrm{DMSO}-d_{6}\right) \delta 141.11$ (C, C5), 138.98 (C, C8), 118.54 (CH, C6), 116.32 (CH, C7), 69.81 (C, C17), $68.47(\mathrm{CH}, \mathrm{C} 3), 52.63\left(\mathrm{CH}_{2}, \mathrm{C} 20\right), 50.00(\mathrm{CH}, \mathrm{C} 14)$, 45.73 (CH, C9), $40.69\left(\mathrm{CH}_{2}, \mathrm{C} 4\right), 39.89(\mathrm{C}, \mathrm{C} 13), 37.82\left(\mathrm{CH}_{2}, \mathrm{C} 1\right), 36.65(\mathrm{C}, \mathrm{C} 10), 33.01\left(\mathrm{CH}_{2}, \mathrm{C} 12\right)$, $31.73\left(\mathrm{CH}_{2}, \mathrm{C} 2\right), 28.31\left(\mathrm{CH}_{2}, \mathrm{C} 16\right), 21.91\left(\mathrm{CH}_{2}, \mathrm{C} 15\right), 20.00\left(\mathrm{CH}_{2}, \mathrm{C} 11\right), 15.98\left(\mathrm{CH}_{3}, \mathrm{C} 19\right), 14.14\left(\mathrm{CH}_{3}\right.$, C18); ESI-MS (+) $m / z 301(\mathrm{M}+\mathrm{H})^{+}, 342\left(\mathrm{M}+\mathrm{CH}_{3} \mathrm{CN}+\mathrm{H}\right)^{+}$.

Solid supported 2 (3). 2 (1.55 g, $5.16 \mathrm{mmol}$ ) was loaded onto macrobeads (3.79 g, approximately 25800 beads) via silyl linker by the reported method. The weight of yielded beads was $4.51 \mathrm{~g}$. The loading level was $140 \mathrm{nmol} / \mathrm{bead}$, which was confirmed by the weight gain and quantification using an UV standard curve $(280 \mathrm{~nm})$ of cleaved material 2 from 10 beads.

3) Optimization of reaction conditions

(3-1) First step (epoxide opening)

(i) Thiols. A mixture of $\mathbf{3}$ (20 beads), a thiol (40 mmol), and THF (0.2 $\mathrm{ml})$ in a $4 \mathrm{ml}$ glass vial was heated at $60{ }^{\circ} \mathrm{C}$ for $40 \mathrm{hr}$. The beads were washed and dried in vacuo.

(ii) Secondary amine. A mixture of 3 (20 beads), $\mathrm{LiClO}_{4}(2.1 \mathrm{mg}, 20 \mathrm{mmol})$, a secondary amine $(60 \mathrm{mmol})$, and THF $(0.2 \mathrm{ml})$ in a $4 \mathrm{ml}$ glass vial was heated at $60{ }^{\circ} \mathrm{C}$ for $40 \mathrm{hr}$. The beads were washed and dried in vacuo.

(iii) Primary amine. A mixture of 3 (20 beads), $\mathrm{LiClO}_{4}(2.1 \mathrm{mg}, 20 \mathrm{mmol})$, a primary amine (160 $\mathrm{mmol})$, and THF $(0.2 \mathrm{ml})$ in a $4 \mathrm{ml}$ glass vial was heated at $60{ }^{\circ} \mathrm{C}$ for $60 \mathrm{hr}$. The beads were washed and dried in vacuo.

(3-2) Second step (amine modification)

(i) Acylation by acyl chloride or chloroformate. A mixture of $\mathbf{1 0}$ (20 beads), DIPEA (10.5 ml, 60 $\mathrm{mmol})$, an acid chloride or a chloroformate $(40 \mathrm{mmol})$, and $\mathrm{CH}_{2} \mathrm{Cl}_{2}(0.2 \mathrm{ml})$ in a $4 \mathrm{ml}$ glass vial was stood at room temperature for $20 \mathrm{hr}$. The beads were washed and dried in vacuo.

(ii) Acylation by sulfonyl chloride. A mixture of $\mathbf{1 0}$ (20 beads), DMAP (7.3 mg, $60 \mathrm{mmol}$ ), a sulfonyl chloride $(40 \mathrm{mmol})$, and $\mathrm{CH}_{2} \mathrm{Cl}_{2}(0.2 \mathrm{ml})$ in a $4 \mathrm{ml}$ glass vial was stood at room temperature for $20 \mathrm{hr}$. The beads were washed and dried in vacuo.

(iii) Urea formation by isocyanate. A mixture of $\mathbf{1 0}$ (20 beads), an isocyanate (40 mmol), and $\mathrm{CH}_{2} \mathrm{Cl}_{2}(0.2 \mathrm{ml})$ in a $4 \mathrm{ml}$ glass vial was stood at room temperature for $20 \mathrm{hr}$. The beads were washed and dried in vacuo.

(iv) b-Hydroxyalkylation by epoxide. A mixture of $\mathbf{1 0}$ (40 beads), an epoxide ( $80 \mathrm{mmol}$ ), and IPA $(0.05 \mathrm{ml})$ in a $4 \mathrm{ml}$ glass vial was heated at $60{ }^{\circ} \mathrm{C}$ for $20 \mathrm{hr}$. The beads were washed and dried in vacuo.

(v) Oxazolidinone formation by 1,1-carbonyldiimidazol. $\mathrm{NaH}(60 \%$ suspension in mineral oil, $0.8 \mathrm{mg}, 20 \mathrm{mmol})$ was added to $\mathbf{1 0}(20$ beads $)$ and THF $(0.2 \mathrm{ml})$ in a $4 \mathrm{ml}$ glass vial. The mixture was stood at room temperature for $15 \mathrm{~min}$. 1,1-Carbonyldiimidazol $(6.5 \mathrm{mg}, 40 \mathrm{mmol})$ was added to the mixture. The mixture was heated at $60{ }^{\circ} \mathrm{C}$ for $20 \mathrm{hr}$. The beads were washed with IPA quickly and a standard protocol, then dried in vacuo.

(3-3) Third step

Before the solid phase chemistry, compound 7 was used to optimize the reaction condition in solution phase, and to confirm the structure of the product.

$(1 R, 3 S, 6 R, 7 R, 10 S, 11 S, 14 S, 15 R)-16-B e n z o y l-6,10-d i m e t h y l-11-[1,4,5-t r i m e t h y l-(2 E)-2-$ hexenyl]pentacyclo[13.2.2.0 $\left.0^{1,6} \cdot 0^{7,15} \cdot 0^{10,14}\right]$ nonadeca-16,18-dien-3-yl acetate (7). A solution of $\mathrm{Et}_{2} \mathrm{AlCl}$ in hexanes $(1 \mathrm{M}$ solution, $5.7 \mathrm{ml}, 5.7 \mathrm{mmol}, 5$ eq.) was added to a solution of ergosterol acetate $(500 \mathrm{mg}, 1.14 \mathrm{mmol})$ and 1-phenyl-2-propyn-1-one (297 mg, $2.28 \mathrm{mmol}, 2 \mathrm{eq}$.$) in$ $\mathrm{CH}_{2} \mathrm{Cl}_{2}(15 \mathrm{ml})$. The mixture was stirred at room temperature for $15 \mathrm{hr}$. The reaction mixture was poured into water $(100 \mathrm{ml})$, extracted with EtOAc $(100 \mathrm{ml} \times 2)$. The combined organic solution was washed with brine $\left(100 \mathrm{ml}\right.$ x 2), dried over $\mathrm{MgSO}_{4}$, then concentrated. Purification by column chromatography (Hexane/EtOAc $=40 / 1)$ followed by recrystallization in hexane $/ \mathrm{CH}_{2} \mathrm{Cl}_{2}$ provided $17(3.10 \mathrm{~g}, 33 \%)$ as white crystals. The structure was confirmed by X- 
ray crystallographic analysis. The details are below.

X-ray crystallographic analysis of 7. Data were collected using a Bruker SMART CCD (charge coupled device) based diffractometer equipped with an LT-2 low-temperature apparatus operating at $213 \mathrm{~K}$. A suitable crystal was chosen and mounted on a glass fiber using grease. Data were measured using omega scans of $0.3^{\circ}$ per frame for 45 seconds, such that a hemisphere was collected. A total of 1271 frames were collected with a maximum resolution of $0.90 \AA$. The first 50 frames were recollected at the end of data collection to monitor for decay. Cell parameters were retrieved using SMART software and refined using SAINT on all observed reflections. Data reduction was performed using the SAINT software which corrects for Lp and decay. Absorption corrections were applied using SADABS supplied by George Sheldrick. The structures are solved by the direct method using the SHELXS-97 program and refined by least squares method on $\mathrm{F}^{2}$, SHELXL-97, incorporated in SHELXTL-PC V 5.10. The structure was solved in the space group P2(1)(\#4) by analysis of systematic absences. All non-hydrogen atoms are refined anisotropically. Hydrogens were calculated by geometrical methods and refined as a riding model. The crystal used for the diffraction study showed no decomposition during data collection. Drawing is done at $50 \%$ ellipsoids.

Crystal data and structure refinement for 7

Empirical formula

Formula weight

$\mathrm{C}_{39} \mathrm{H}_{52} \mathrm{O}_{3}$

Temperature

568.81

Wavelength

213 (2) K

Crystal system

$0.71073 \AA$

Space group

Monoclinic

Unit cell dimensions

Volume, z

Density (calculated)

$\mathrm{P} 21$

$$
\begin{aligned}
& a=14.251(1) \AA \text { alpha }=90^{\circ} \\
& b=6.4802(5) \AA \text { beta }=97.679(3)^{\circ} \\
& c=19.219(1) \AA \text { gamma }=90^{\circ} \\
& 1759.0(2) \AA^{3}, 2 \\
& 1.074 \mathrm{mg}^{3} \mathrm{~m}^{3} \\
& 0.066 \mathrm{~mm}^{-1} \\
& 620 \\
& 0.25 \times 0.10 \times 0.10 \mathrm{~mm} \\
& 1.44 \text { to } 22.49^{\circ} \\
& -15 \leq h \leq 13,-6 \leq k \leq 6,-19 \leq l \leq 19 \\
& 5528 \\
& 3072\left(\mathrm{R}_{\text {int }}=0.0738\right) \\
& 87.5 \% \\
& \text { None } \\
& \text { Full-matrix least-squares on } \mathrm{F}^{2} \\
& 3072 / 1 / 379 \\
& 1.023 \\
& \mathrm{R} 1=0.0697, \text { wR } 2=0.1737 \\
& \mathrm{R} 1=0.1032, \text { wR } 2=0.1952 \\
& -2(4) \\
& 0.435 \text { and }-0.240 \text { e } \AA^{-3}
\end{aligned}
$$$$
\mathrm{F}(000)
$$$$
\text { q range for data collection }
$$$$
\text { Reflections collected }
$$$$
\text { Independent reflections }
$$$$
\text { Completeness to } \mathrm{q}=22.49^{\circ}
$$$$
\text { Absorption correction }
$$$$
\text { Refinement method }
$$$$
\text { Data / restraints / parameters }
$$$$
\text { Goodness-of fit on F2 }
$$$$
\text { Final } R \text { indices [I }>2 s(I)]
$$$$
\mathrm{R} \text { indices (all data) }
$$$$
\text { Absolute structure parameter }
$$$$
\text { Largest diff. Peak and hole }
$$ 
Atomic coordinates $\left[x \quad 10^{4}\right]$ and equivalent isotropic displacement parameters $\left[\dot{A}^{2} \times 10^{3}\right]$ for 17 , U(aq) is defined as one third of the trace of the orthogonalized $v_{i j}$ tensor.

\begin{tabular}{|c|c|c|c|c|}
\hline & $\mathbf{x}$ & $y$ & $z$ & $\mathrm{v}(\mathrm{eq})$ \\
\hline$o(1)$ & $-4163(3)$ & $-4695(7)$ & $861(3)$ & $56(1)$ \\
\hline$O(2)$ & $-4260\{4\}$ & $-8085(9)$ & $1060(5)$ & $103(2)$ \\
\hline$O(3)$ & $485(3)$ & $-5864(6)$ & $3115(3)$ & $55(1)$ \\
\hline$C(1)$ & $-1205(4)$ & $-3045(8)$ & $1029(4)$ & $36(2)$ \\
\hline$c(2)$ & $-1521(4)$ & $-5247(9)$ & $790(4)$ & $37(2)$ \\
\hline$c(3)$ & $-2579(4)$ & $-5475(9)$ & $558(4)$ & $46(2)$ \\
\hline$C(4)$ & $-3149(4)$ & $-4750(10)$ & $1123(4)$ & $46(2)$ \\
\hline$C(5)$ & $-2892(4)$ & $-2541(9)$ & $1374(4)$ & $45(2)$ \\
\hline$c(6)$ & $-1628(4)$ & $-2351(9)$ & $1603(4)$ & $39(2)$ \\
\hline$c(7)$ & $-1509(4)$ & $-3625(9)$ & $2256(4)$ & $36(2)$ \\
\hline$C(B)$ & $-607(4)$ & $-3470(8)$ & $2529(4)$ & $32(2)$ \\
\hline$c(9)$ & $-36(4)$ & $-2030(9)$ & $2115(4)$ & $37(2)$ \\
\hline$C(10)$ & $1006(4)$ & $-1893(8)$ & $2392(4)$ & $39(2)$ \\
\hline$C(11)$ & $1286(4)$ & $-889(10)$ & $3121(4)$ & $47(2)$ \\
\hline$c(12)$ & $2361(5)$ & $-515(11)$ & $3144(4)$ & $57(2)$ \\
\hline$C(13)$ & $2643(4)$ & $-1213(10)$ & $2433(4)$ & $46(2)$ \\
\hline$C(14)$ & $2704(4)$ & $-990(9)$ & $1923(4)$ & $41(2)$ \\
\hline$C(15)$ & $1575(5)$ & $-2233(10)$ & $1240(4)$ & $49(2)$ \\
\hline$c(16)$ & $561(4)$ & $-2220(30)$ & $902(4)$ & $43(2)$ \\
\hline$c(17)$ & $-147(4)$ & $-3057(9)$ & $1350(4)$ & $37(2)$ \\
\hline$c(18)$ & $-585(4)$ & $-7(9)$ & $2046(4)$ & $38(2)$ \\
\hline$C(19)$ & $-1491(5)$ & $-183(8)$ & $1808(4)$ & $39(2)$ \\
\hline$C(20)$ & $-4653(6)$ & $-6424(15)$ & $873(6)$ & $78(3)$ \\
\hline$C(21)$ & $-5654(5)$ & $-6166(14)$ & $578(6)$ & $84(3)$ \\
\hline$C(22)$ & $-218(4)$ & $-4774(9)$ & $3140(4)$ & $41(2)$ \\
\hline$c(23)$ & $-701(4)$ & $-4719(11)$ & $3774(4)$ & $43(2)$ \\
\hline$C(24)$ & $-1216(5)$ & $-3004(13)$ & $3953(4)$ & $59(2)$ \\
\hline$C(25)$ & $-1635(6)$ & $-2933(18)$ & $4553(6)$ & $81(3)$ \\
\hline$c(26)$ & $-1557(7)$ & $-4620(30)$ & $4989(6)$ & $115(4)$ \\
\hline$c(27)$ & $-1057(8)$ & $-6360(20)$ & $4834(6)$ & $108(4)$ \\
\hline$C(28)$ & $-621(5)$ & $-6370(13)$ & $4247(5)$ & $69(2)$ \\
\hline$c(29)$ & $-1399(5)$ & $-1610(10)$ & $386(4)$ & $46(2)$ \\
\hline$C(30)$ & $1522(5\rangle$ & $1352(9)$ & $1740(4)$ & $46(2)$ \\
\hline$c(31)$ & $3554\langle 5\rangle$ & $-165(12)$ & $2232(5)$ & $55(2)$ \\
\hline$C(32)$ & $3935(5)$ & $-1236(16)$ & $1622(5)$ & $86(3)$ \\
\hline$c(33)$ & $4298(5)$ & $-152(12)$ & $2858(5)$ & $65(2)$ \\
\hline$c(34)$ & $4684(5)$ & $1514(12)$ & $318 \mathrm{~B}(5)$ & $66(2)$ \\
\hline$c(35)$ & $5431(6)$ & $1535(13)$ & $3822(5)$ & $70(2)$ \\
\hline$C(36)$ & $5063(9)$ & $2590(20)$ & $4421(7)$ & $130(5)$ \\
\hline$C(37)$ & $6355(6)$ & $2510(14)$ & $3658(6)$ & $87(3)$ \\
\hline$c(38)$ & $6245(7)$ & $4751(16)$ & $3397(7)$ & $115(4)$ \\
\hline$C(39)$ & $6829(6)$ & $1188(17)$ & $3158(6)$ & $101(4)$ \\
\hline
\end{tabular}


Bond lengths [Aं] and angles $\left[{ }^{\circ}\right]$ for 17 .

\begin{tabular}{|c|c|c|c|}
\hline$O(1)-C(20)$ & $1.322(9 !$ & $O(1)-C(4)$ & $1.465(7)$ \\
\hline$O(2)-C(20)$ & 1.244 (11), & $o(3)-c(22)$ & $1.232(7)$ \\
\hline$C(1)-C(29)$ & $1.541(10)$ & $C(1)-C(2)$ & $1.547(9)$ \\
\hline$C(1)-C(17)$ & $1.551(9)$ & $C(1)-C(6)$ & $1.571(9)$ \\
\hline$C(2)-C(3)$ & $1.3=1(8)$ & $C(3)-C(4)$ & $1.515(9)$ \\
\hline$C(4)-C(5)$ & $1.539(9)$ & $c(5)-c(6)$ & $1.527(9)$ \\
\hline$C(6)-C(19)$ & $1.519(8)$ & $c(6)-c(7)$ & $1.521(10)$ \\
\hline$C(7)-C(8)$ & $1.324(8)$ & $c(8)-C(22)$ & $1.492(10)$ \\
\hline$c(8)-c(9)$ & $1.529(8)$ & $c(\therefore-c(10)$ & $1.511(9)$ \\
\hline$c(9)-C(18)$ & $1.524(8)$ & $c(9) \cdot C: 1.7)$ & $1.606(10)$ \\
\hline$C(10)-C(14)$ & $1.544(8)$ & $c(10)-=\left(1 i_{i}\right)$ & $1.548(10)$ \\
\hline$C(11)-C(12)$ & $1.547(8)$ & $C(12)-C: 13)$ & $1.544(10)$ \\
\hline$C(13)-C(14)$ & $1.557(10)$ & $c(13)-c(3:)$ & $1.559(8)$ \\
\hline$C(14)-C(15)$ & $1.530(10)$ & $C(14)-C(30)$ & $1.572(8)$ \\
\hline$C(15)-C(16)$ & $1.504(10)$ & $C(16)-C(17)$ & $1.513(8)$ \\
\hline$C(18)-C(19)$ & $1.316(9)$ & $C(20)-C(21)$ & $1.472(12)$ \\
\hline$C(22)-C(23)$ & $1.479(9)$ & $C(23)-C(28)$ & $1 . \therefore 98(11)$ \\
\hline$C(23)-C(24)$ & $1.399(10)$ & $c(24)-c(25)$ & $1.365(12)$ \\
\hline$C(25)-C(26)$ & $1.375(17)$ & $C(26)-C(27)$ & $\left.1.385^{\prime} 18\right)$ \\
\hline$C(27)-C(28)$ & $1.360(13)$ & $c(31)-c(33)$ & $\therefore .493(1 ?)$ \\
\hline$C(31)-C(32)$ & $1.524(11)$ & $c(33)-c(34)$ & $1.733(11)$ \\
\hline$c(34)-c(35)$ & $1.507(12)$ & $C(35)-C(36)$ & $1.453(14)$ \\
\hline$C(35)-C(37)$ & $1.532(10)$ & $c(37)-c(39)$ & $1.511: 12)$ \\
\hline$C(37)-C(38)$ & $1.537(14)$ & & \\
\hline$C(20)-O(1)-c(4)$ & $118.2(6)$ & $C(29)-C(1)-C(2)$ & $107.7(6)$ \\
\hline$c(29)-C(1)-C(17)$ & $112.6(5)$ & $C(2)-C(1)-C(17)$ & $109.7(4)$ \\
\hline$C(29)-C(1)-C(6)$ & $109.5(5)$ & $c(2)-c(1)-C(6)$ & $107.7(5)$ \\
\hline$c(17)-c(1)-c(6)$ & $109.5(6)$ & $C(3)-C(2)-C(1)$ & $114.4(5)$ \\
\hline$C(4)-C(3)-C(2)$ & $.111 .5(6)$ & $O(1)-C(4)-C(3)$ & $111.1(6)$ \\
\hline$O(1)-C(4)-C(5)$ & $105.5(5)$ & $C(3)-C(4)-C(5)$ & $112.7(5)$ \\
\hline$C(6)-C(5)-C(4)$ & $110.5(5)$ & $C(19)-C(6)-C(7)$ & $103.8(6)$ \\
\hline$C(19)-C(6)-C(5)$ & $114.6(5)$ & $c(7)-c(6)-c(5)$ & $111.7(5)$ \\
\hline$C(19)-C(6)-C(1)$ & $105.0(5)$ & $C(7)-C(6)-C(1)$ & $106.9(5)$ \\
\hline$c(5)-c(6)-c(1)$ & $114.0(6)$ & $C(8)-C(7)-C(6)$ & $116.9(5)$ \\
\hline$c(7)-c(8)-c(22)$ & $120.5(5)$ & $C(7)-C(8)-C(9)$ & $113.4(6)$ \\
\hline$C(22)-C(8)-C(9)$ & $125.9(5)$ & $c(10)-c(9)-C(18)$ & $116.7(5)$ \\
\hline$C(10)-C(9)-C(8)$ & $114.8(6)$ & $C(18)-C(9)-C(8)$ & $105.5(4)$ \\
\hline$c(10)-c(9)-c(17)$ & $108.6(4)$ & $C(18)-c(9)-C(17)$ & $107.0(5)$ \\
\hline$C(8)-c(9)-c(17)$ & $103.1(5)$ & $C(9)-C(10)-C(14)$ & $119.8(6)$ \\
\hline$c(9)-c(10)-c(11)$ & $117.7(5)$ & $C(14)-C(10)-C(11)$ & $105.1(5)$ \\
\hline$c(12)-c(11)-C(10)$ & $103.1(5)$ & $c(13)-c(12)-c(11)$ & $107.6(6)$ \\
\hline$C(12)-C(13)-C(14)$ & $103.1(5)$ & $c(12)-c(13)-c(31)$ & $114.5(6)$ \\
\hline$C(14)-C(13)-C(31)$ & $118.6(6)$ & $C(15)-C(14)-C(10)$ & $106.8(5)$ \\
\hline$c(15)-c(14)-c(13)$ & $119.1(5)$ & $C(10)-C(14)-C(13)$ & $99.4(6)$ \\
\hline$C(15)-C(14)-C(30)$ & $108.6(6)$ & $c(10)-c(14)-c(30)$ & $113.4(5)$ \\
\hline$c(13)-c(14)-c(30)$ & $109.4(5)$ & $C(16)-C(15)-C(14)$ & $111.4(5)$ \\
\hline$C(15)-C(16)-C(17)$ & $115.3(6)$ & $c(16)-c(17)-c(1)$ & $117.2(6)$ \\
\hline$c(16)-c(17)-c(9)$ & $112.1(5)$ & $c(1)-c(17)-c(9)$ & $109.4(4)$ \\
\hline$c(19)-c(18)-c(9)$ & $115.1(5)$ & $c(18)-c(19)-c(6)$ & $115.5(5)$ \\
\hline$O(2)-C(20)-O(1)$ & $121.3(7)$ & $O(2)-C(20)-C(21)$ & $125.8(7)$ \\
\hline$O(1)-C(20)-C(21)$ & $112.6(8)$ & $o(3)-c(22)-c(23)$ & $121.1(6)$ \\
\hline$O(3)-C(22)-C(8)$ & $120.9(6)$ & $c(23)-c(22)-C(8)$ & $118.0(5)$ \\
\hline$C(28)-C(23)-C(24)$ & $116.7(6)$ & $C(28)-C(23)-C(22)$ & $120.7(7)$ \\
\hline$C(24)-C(23)-C(22)$ & $122.6(6)$ & $C(25)-C(24)-C(23)$ & $122.4(8)$ \\
\hline$C(24)-C(25)-C(26)$ & $118.5(10)$ & $C(25)-C(26)-C(27)$ & $121.3(9)$ \\
\hline$C(28)-C(27)-C(26)$ & $119.1(10)$ & $C(27)-C(28)-C(23)$ & $121.9(9)$ \\
\hline$c(33)-c(31)-c(32)$ & $109.5(6)$ & $c(33)-c(31)-c(13)$ & $109.2(7)$ \\
\hline$c(32)-c(31)-c(13)$ & $112.7(6)$ & $C(34)-C(33)-C(31)$ & $126.2(8)$ \\
\hline$c(33)-c(34)-c(35)$ & $126.5(8)$ & $c(36)-c(35)-c(34)$ & $110.2(8)$ \\
\hline$c(36)-c(35)-c(37)$ & $111.7(8)$ & $c(34)-c(35)-c(37)$ & $111.8(8)$ \\
\hline$c(39)-c(37)-c(35)$ & $111.5(8)$ & $c(39)-C(37)-C(38)$ & $111.3(10)$ \\
\hline$c(35)-c(37)-c(38)$ & $113.8(8)$ & & \\
\hline
\end{tabular}

Symmetry transformations used to generate equivalent atoms: 
Anisotropic displacement parameters $\left[\dot{A}^{2} \times 10^{3}\right]$ for 17 .

The anisotropic displacement factor exponent takes the form: $-2 \pi^{2}\left[\left(\mathrm{ha}^{*}\right)^{2} \mathrm{u}_{11}+\ldots+2 \mathrm{hka}{ }^{*} \mathrm{~b}^{*} \mathrm{u}_{12}\right]$

$\begin{array}{llllll}\mathrm{U11} & \mathrm{U} 22 & \mathrm{U} 33 & \mathrm{U} 23 & \mathrm{U13} & \mathrm{U12}\end{array}$

\begin{tabular}{|c|c|c|c|c|c|c|}
\hline$O(1)$ & $53(3)$ & $45(3)$ & $64(5)$ & $7(3)$ & $-12(2)$ & $-8(2)$ \\
\hline$O(2)$ & $83(4)$ & $53(4)$ & $165(8)$ & $15(4)$ & $-12(4)$ & $-12(3)$ \\
\hline$O(3)$ & $69(3)$ & $40(3)$ & $54(4)$ & $5(2)$ & $4(3)$ & $10(2)$ \\
\hline$C(1)$ & $52(4)$ & $28(3)$ & $29(5)$ & $7(3)$ & $4(3)$ & $4(3)$ \\
\hline$c(2)$ & $48(4)$ & $35(4)$ & $26(5)$ & $-1(3)$ & $-3(3)$ & $-3(3)$ \\
\hline$c(3)$ & $53(4)$ & $43(4)$ & $37(6)$ & $-2(3)$ & $-8(4)$ & $0(3)$ \\
\hline$C(4)$ & $40(4)$ & $41(4)$ & $49(6)$ & $-2(4)$ & $-16(3)$ & $-2(3)$ \\
\hline$C(5)$ & $45(4)$ & $40(4)$ & $47(6)$ & $5(3)$ & $-4(3)$ & $-2(3)$ \\
\hline$C(6)$ & $52(4)$ & $27(3)$ & $34(6)$ & $1(3)$ & $-1(3)$ & $- \pm(3)$ \\
\hline$C(7)$ & $47(4)$ & $31(3)$ & $29(5)$ & $1\langle 3\rangle$ & $-1(3)$ & $-1(3)$ \\
\hline$c(8)$ & $47(4)$ & $22(3)$ & $28(5)$ & $-3(3)$ & $6(3)$ & $I(3)$ \\
\hline$c(9)$ & $46(4)$ & $34(4)$ & $31(6)$ & $-3(3)$ & $1(3)$ & $-1(3)$ \\
\hline$c(10)$ & $48(4)$ & $25(3)$ & $41(6)$ & $-3(3)$ & $0(4)$ & $-1(3)$ \\
\hline$c(1.1)$ & $58(4)$ & $48(4)$ & $32(6)$ & $-3(3)$ & $0(3)$ & $-9(3)$ \\
\hline$c(12)$ & $57(4)$ & $70(5)$ & $42(6)$ & $-10(4)$ & $-1(4)$ & $-11(3)$ \\
\hline$c(13)$ & $51(4)$ & $40(4)$ & $47(6)$ & $-5(3)$ & $4(4)$ & $-8(3)$ \\
\hline$c(14)$ & $52(4)$ & $40(4)$ & $30(5)$ & $-7(3)$ & $3(3)$ & $-8(3)$ \\
\hline$c(15)$ & $63(4)$ & $48(4)$ & $38(6)$ & $-4(3)$ & $10(4)$ & $-4(3)$ \\
\hline$c(16)$ & $53(4)$ & $43(4)$ & $32(6)$ & $-11(3)$ & $4(4)$ & $-6(3)$ \\
\hline$c(17)$ & $46(4)$ & $28(3)$ & $38(6)$ & $-3(3)$ & $6(3)$ & $-2(3)$ \\
\hline$c(18)$ & $51(4)$ & $26(3)$ & $37(5)$ & $-1(3)$ & $3(3)$ & $-7(3)$ \\
\hline$C(19)$ & $59(4)$ & $25(3)$ & $32(5)$ & $-5(3)$ & $-3(3)$ & $5(3)$ \\
\hline$c(20)$ & $74(6)$ & $68(6)$ & $85(9)$ & $0(5)$ & $-13(5)$ & $-24(5)$ \\
\hline$c(21)$ & $55(5)$ & $72(5)$ & $116(10)$ & $-19(5)$ & $-17(5)$ & $-15(4)$ \\
\hline$C(22)$ & $45(4)$ & $29(3)$ & $47(6)$ & $-2(3)$ & $2(3)$ & $-7(3)$ \\
\hline$C(23)$ & $43(4)$ & $53(4)$ & $30(6)$ & $I 1(4)$ & $-4(3)$ & $-6(3)$ \\
\hline$C(24)$ & $52(4)$ & $85(6)$ & $38(6)$ & $10(4)$ & $4(4)$ & $-7(4)$ \\
\hline$C(25)$ & $69(5)$ & $136(9)$ & $38(8)$ & $1(6)$ & $4(5)$ & $12(5)$ \\
\hline$C(26)$ & $63(6)$ & $224(15)$ & $58(10)$ & $15(10)$ & $5(6)$ & $-12(8)$ \\
\hline$C(27)$ & $94(7)$ & $165(11)$ & $64(10)$ & $67(8)$ & $1(6)$ & $-36(7)$ \\
\hline$C(28)$ & $70(5)$ & $81(6)$ & $52(7)$ & $25(5)$ & $-8(5)$ & $-13(4)$ \\
\hline$C(29)$ & $56(4)$ & $45(4)$ & $36(6)$ & $2(3)$ & $-3(3)$ & $-6(3)$ \\
\hline$c(30)$ & $68(4)$ & $37(4)$ & $32(5)$ & $-1(3)$ & $-1(4)$ & $-5(3)$ \\
\hline$c(31)$ & $51(4)$ & $65(5)$ & $47(6)$ & $-10(4)$ & $5(4)$ & $-11(3)$ \\
\hline$C(32)$ & $63(5)$ & $120(7)$ & $7 y(8)$ & $-12(6)$ & $17(5)$ & $-27(5)$ \\
\hline$c(33)$ & $62(5)$ & $68\langle 5\rangle$ & $64(8)$ & $4(5)$ & $9(5)$ & $-3(4)$ \\
\hline$C(34)$ & $52(4)$ & $59\langle 5\rangle$ & $80(8)$ & $5(5)$ & $-13(4)$ & $-7(4)$ \\
\hline$c(35)$ & $74(5)$ & $74(5)$ & $59(8)$ & $-1(5)$ & $2(5)$ & $-16\langle 4\rangle$ \\
\hline$c(36)$ & $151(10)$ & $164(12)$ & $72(12)$ & $0(8)$ & $5(8)$ & $-4 I(9)$ \\
\hline$c(37)$ & $76(6)$ & $87(7)$ & $91(9)$ & $-6\langle 6\rangle$ & $-12(6)$ & $-14(5)$ \\
\hline$C(38)$ & $104(7)$ & $100(8)$ & $140(13)$ & $4\langle 7\rangle$ & $13(8)$ & $-39(6)$ \\
\hline$C(39)$ & $66(6)$ & $128(9)$ & $205(11)$ & $-6\langle 7\rangle$ & $-2(6)$ & $0(5)$ \\
\hline
\end{tabular}


Hydrogen coordinates $\left(\times 10^{4}\right)$ and isotropic disk lacement parameters $\left(\dot{A}^{2} \times 10^{3}\right)$ for 17 .

\begin{tabular}{|c|c|c|c|c|}
\hline & $\mathbf{x}$ & $\mathbf{v}$ & z & $\mathrm{U}(\mathrm{eq})$ \\
\hline$H(2 A)$ & -1183 & -5650 & 329 & 45 \\
\hline $\mathrm{F}(2 \mathrm{~B})$ & -1333 & -6206 & 1178 & 45 \\
\hline $\mathrm{H}(3 \mathrm{~A})$ & -2758 & -4668 & 130 & 55 \\
\hline $\mathrm{H}(3 \mathrm{~B})$ & -2726 & -6927 & 449 & $\equiv 5$ \\
\hline$H(4 A)$ & -3041 & -5703 & 1528 & 55 \\
\hline$H(5 A)$ & -3087 & -1563 & 993 & 54 \\
\hline $\mathrm{H}(5 \mathrm{~B})$ & -3232 & -2195 & 1769 & 54 \\
\hline$E(7 A)$ & -1931 & -4483 & 2458 & 43 \\
\hline$H(10 A)$ & 1206 & -3349 & 2461 & 46 \\
\hline$H(11 A)$ & 946 & 412 & 3160 & 56 \\
\hline$H(11 B)$ & 1155 & -1818 & 3499 & 56 \\
\hline$H(12 A)$ & 2507 & 952 & 3222 & 69 \\
\hline $\mathrm{H}(12 \mathrm{~B})$ & 2713 & -1308 & 3528 & 69 \\
\hline$H(13 A)$ & 2780 & -2709 & 2475 & 55 \\
\hline$H(15 A)$ & 1977 & -1647 & 914 & 59 \\
\hline$H(15 B)$ & 1778 & -3660 & 1339 & 59 \\
\hline$H(16 A)$ & 514 & -3027 & 468 & 51 \\
\hline $\mathrm{H}(16 \mathrm{~B})$ & 383 & -796 & 773 & 51 \\
\hline$H(17 A)$ & 20 & -4539 & 1428 & 45 \\
\hline $\mathrm{H}(18 \mathrm{~A})$ & -298 & 1274 & 2165 & 46 \\
\hline$H(19 A)$ & -1903 & 957 & 1767 & 47 \\
\hline$H(21 A)$ & -5985 & -7465 & 607 & 125 \\
\hline$H(21 B)$ & -5695 & -5742 & 91 & 125 \\
\hline$H(21 C)$ & -5941 & -5121 & 843 & 125 \\
\hline$H(24 A)$ & -1276 & -1860 & 3649 & 70 \\
\hline$H(25 A)$ & -1969 & -1754 & 4664 & 98 \\
\hline$H(26 A)$ & -1848 & -4601 & 5400 & 139 \\
\hline$H(27 A)$ & -1021 & -7519 & 5132 & 130 \\
\hline$H(28 A)$ & -254 & -7520 & 4156 & 83 \\
\hline$H(29 A)$ & -1014 & -2038 & 31 & 70 \\
\hline $\mathrm{H}(29 \mathrm{~B})$ & -1240 & -202 & 527 & 70 \\
\hline$H(29 C)$ & -2063 & -1687 & 195 & 70 \\
\hline $\mathrm{H}(30 \mathrm{~A})$ & 933 & 1494 & 1427 & 69 \\
\hline $\mathrm{H}(3 \mathrm{OB})$ & 2039 & 1887 & 1512 & 69 \\
\hline $\mathrm{H}(30 \mathrm{C})$ & 1482 & 2122 & 2168 & 69 \\
\hline$H(31 A)$ & 3400 & 1282 & 2096 & 65 \\
\hline $\mathrm{H}(32 \mathrm{~A})$ & 4495 & -515 & 1514 & 129 \\
\hline $\mathrm{H}(32 \mathrm{~B})$ & 3455 & -1222 & 1213 & 129 \\
\hline $\mathrm{H}(32 \mathrm{C})$ & 4099 & -2651 & 1750 & 129 \\
\hline$H(33 A)$ & 4516 & -1442 & 3036 & 77 \\
\hline$H(34 A)$ & 4468 & 2803 & 3008 & 79 \\
\hline$H(35 A)$ & 5568 & 85 & 3961 & 84 \\
\hline$H(36 A)$ & 4478 & 1938 & 4509 & 195 \\
\hline$H(36 B)$ & 4946 & 4033 & 4306 & 195 \\
\hline$H(36 C)$ & 5527 & 2486 & 4837 & 195 \\
\hline$H(37 A)$ & 6789 & 2547 & 4105 & 104 \\
\hline$H(38 A)$ & 6857 & 5284 & 3316 & 173 \\
\hline $\mathrm{H}(38 \mathrm{~B})$ & 5994 & 5589 & 3747 & 173 \\
\hline$H(38 C)$ & 5813 & 4793 & 2962 & 173 \\
\hline H(39) & 6920 & -195 & 3349 & 151 \\
\hline H (39B) & 7437 & 2791 & 3098 & 151 \\
\hline $\mathrm{H}(39 \mathrm{C})$ & 6432 & 1129 & 2707 & 151 \\
\hline
\end{tabular}


Diels-Alder reaction on solid phase. A solution of ynone $(20 \mathrm{mmol})$ in $\mathrm{CH}_{2} \mathrm{Cl}_{2}(0.4 \mathrm{ml})$ was added to 9 (20 beads) under $\mathrm{N}_{2}$. After 30 min a solution of $\mathrm{Et}_{2} \mathrm{AlCl}$ in hexanes ( $1 \mathrm{M}$ solution, $80 \mathrm{ml}, 80 \mathrm{mmol}$ ) was added to the mixture. The mixture was stood at room temperature for $4 \mathrm{hr}$. The reaction solution exchanged to fresh $\mathrm{CH}_{2} \mathrm{Cl}_{2}$ three times under $\mathrm{N}_{2}$. The beads were washed and dried in vacuo.

(3-4) Fourth step

Retro-Diels-Alder reaction. 9 (20 beads) was heated at $110{ }^{\circ} \mathrm{C}$ for $1.5 \mathrm{hr}$ under $\mathrm{N}_{2}$.

4) Ynone synthesis

All ynones used in this paper except commercially available 3-butyn-2-one were synthesized by reported methods via acid chloride (method A) or Weinreb amide (method B).<smiles>C#CC(=O)/C(C)=C/I</smiles>

(E)-3-Methyl-4-oxo-2-hexen-5-yne. It was synthesized by method A: IR (thin film) 3252, 2956, 2094, 1637, $1262 \mathrm{~cm}^{-1}$; ${ }^{1} \mathrm{H}$ NMR (500 MHz, $\mathrm{CDCl}_{3}$ ) $\delta 7.30$ (q, 1H, J = $\left.7.3 \mathrm{~Hz}, \mathrm{C} 2-\mathrm{H}\right), 3.20$ (s, 1H, C6-H), 1.96 $\left(\mathrm{d}, 3 \mathrm{H}, J=7.3 \mathrm{~Hz}, \mathrm{C} 1-\mathrm{H}_{3}\right), 1.82\left(\mathrm{~s}, 3 \mathrm{H}, \mathrm{C} 7-\mathrm{H}_{3}\right)$; ESI-MS $(+) \mathrm{m} / z .131(\mathrm{M}+\mathrm{Na})^{+}$.<smiles>C#CC(=O)c1ccccc1</smiles>

1-Phenyl-2-propyn-1-one. It was synthesized by method A: IR (thin film) 3235, 2092, $1637 \mathrm{~cm}^{-1} ;{ }^{1} \mathrm{H}$ NMR $\left(500 \mathrm{MHz}, \mathrm{CDCl}_{3}\right) \delta 8.18(\mathrm{~d}, 2 \mathrm{H}, J=8.1 \mathrm{~Hz}, \mathrm{C} 5-\mathrm{H}, \mathrm{C} 9-\mathrm{H}), 7.65(\mathrm{t}, 1 \mathrm{H}, J=7.3 \mathrm{~Hz}, \mathrm{C} 7-\mathrm{H}), 7.52(\mathrm{t}$, $2 \mathrm{H}, J=7.8 \mathrm{~Hz}, \mathrm{C} 6-\mathrm{H}, \mathrm{C} 8-\mathrm{H}), 3.44(\mathrm{~s}, 3 \mathrm{H}, \mathrm{C} 3-\mathrm{H})$; ESI-MS (+) $m / z 172\left(\mathrm{M}_{+} \mathrm{CH}_{3} \mathrm{CN}+\mathrm{H}\right)^{+}, 194$ $\left(\mathrm{M}+\mathrm{CH}_{3} \mathrm{CN}+\mathrm{Na}\right)^{+}$.<smiles>C#CC(=O)c1ccc(F)c(F)c1</smiles>

1-(3,4-Difluorophenyl)-2-propyn-1-one. It was synthesized by method A: IR (thin film) 3251, 2099, 1658, 1513, $1285 \mathrm{~cm}^{-1}$; ${ }^{1} \mathrm{H}$ NMR $\left(500 \mathrm{MHz} \mathrm{CDCl}_{3}\right) \delta 8.00-7.96(\mathrm{~m}, 2 \mathrm{H}, \mathrm{C} 5-\mathrm{H}, \mathrm{C} 9-\mathrm{H}), 7.34-7.29$ (m, $1 \mathrm{H}, \mathrm{C} 8-\mathrm{H}), 3.49$ (s, 1H, C3-H); ESI-MS (+) $\mathrm{m} / z 189(\mathrm{M}+\mathrm{Na})^{+}$.

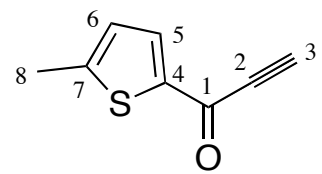

1-(5-Methylthiophen-2-yl)-2-propyn-1-one. It was synthesized by method A: IR (thin film) 3225, 2093, $1604,1447,1284 \mathrm{~cm}^{-1} ;{ }^{1} \mathrm{H}$ NMR $\left(500 \mathrm{MHz}, \mathrm{CDCl}_{3}\right) \delta 7.81(\mathrm{~d}, 1 \mathrm{H}, J=3.9 \mathrm{~Hz}, \mathrm{C} 5-\mathrm{H}), 6.86(\mathrm{dd}, 1 \mathrm{H}, J=$ 3.9, $1.0 \mathrm{~Hz}, \mathrm{C} 6-\mathrm{H}), 3.30(\mathrm{~s}, 1 \mathrm{H}, \mathrm{C} 3-\mathrm{H}), 2.58\left(\mathrm{~s}, 3 \mathrm{H}, \mathrm{C} 8-\mathrm{H}_{3}\right)$; ESI-MS (+) $\mathrm{m} / \mathrm{z}, 151(\mathrm{M}+\mathrm{H})^{+}$.

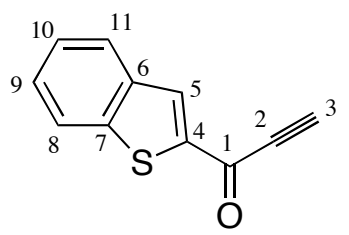

1-(2-Benzo[b]thiophenyl)-2-propyn-1-one. It was synthesized by method A: IR (thin film) 3225, 2093, $1616 \mathrm{~cm}^{-1}$; ${ }^{1} \mathrm{H}$ NMR $\left(500 \mathrm{MHz}, \mathrm{CDCl}_{3}\right) \delta 8.27(\mathrm{~s}, 1 \mathrm{H}, \mathrm{C} 5-\mathrm{H}), 7.95(\mathrm{~d}, 1 \mathrm{H}, J=7.8 \mathrm{~Hz}, \mathrm{C} 8-\mathrm{H}$ or C11-H), $7.89(\mathrm{dd}, 1 \mathrm{H}, J=7.8,1.0 \mathrm{~Hz}, \mathrm{C} 8-\mathrm{H}$ or $\mathrm{C} 11-\mathrm{H}), 7.53(\mathrm{td}, 1 \mathrm{H}, J=7.6,1.5 \mathrm{~Hz}, \mathrm{C} 9-\mathrm{H}$ or C10-H), $7.45(\mathrm{td}$, $1 \mathrm{H}, J=7.6,1.0 \mathrm{~Hz}, \mathrm{C} 9-\mathrm{H}$ or $\mathrm{C} 10-\mathrm{H}), 3.45(\mathrm{~s}, 1 \mathrm{H}, \mathrm{C} 3-\mathrm{H})$; ESI-MS $(+) m / z 187(\mathrm{M}+\mathrm{H})^{+}, 228$ 
$\left(\mathrm{M}+\mathrm{CH}_{3} \mathrm{CN}+\mathrm{H}\right)^{+}$.

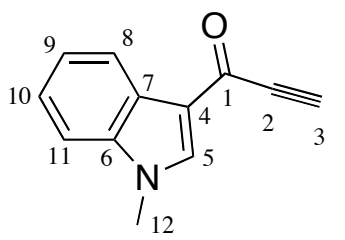

1-(1-Methylindol-3-yl)-2-propyn-1-one. It was synthesized by method A: IR (thin film) 3243, 3112, 2091, 1607, 1526, 1464, 1378, 1089, $929 \mathrm{~cm}^{-1}$; ${ }^{1} \mathrm{H}$ NMR (500 MHz, CDCl ${ }_{3}$ ) $8.39-8.37$ (m, 1H, C8-H), 7.96 (s, 1H, C5-H), 7.37-7.35 (m, 3H, C9-H, C10-H, C11-H), 3.90 (s, 3H, C12-H $), 3.17$ (s, 1H, C3-H); ESI-MS (+) $m / z, 184(\mathrm{M}+\mathrm{H})^{+}$.

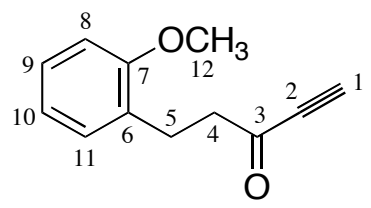

5-(2-Methoxyphenyl)-1-pentyn-3-one. It was synthesized by method A: IR (thin film) 3243, 3112, 2090, 1606, 1465, $929 \mathrm{~cm}^{-1}$; ${ }^{1} \mathrm{H}$ NMR $\left(500 \mathrm{MHz}, \mathrm{CDCl}_{3}\right) \delta 7.22(\mathrm{td}, 1 \mathrm{H}, J=7.8,2.0 \mathrm{~Hz}, \mathrm{C} 9-\mathrm{H}), 7.15(\mathrm{dd}, 1 \mathrm{H}, J$ $=7.3,1.5 \mathrm{~Hz}, \mathrm{C} 11-\mathrm{H}), 6.89(\mathrm{t}, 1 \mathrm{H}, J=7.6 \mathrm{~Hz}, \mathrm{C} 10-\mathrm{H}), 6.86(\mathrm{~d}, 1 \mathrm{H}, J=8.3 \mathrm{~Hz}, \mathrm{C} 8-\mathrm{H}), 3.84(\mathrm{~s}, 3 \mathrm{H}, \mathrm{C} 12-$ $\left.\mathrm{H}_{3}\right), 3.22(\mathrm{~s}, 1 \mathrm{H}, \mathrm{C} 1-\mathrm{H}), 2.99\left(\mathrm{t}, 2 \mathrm{H}, J=7.1 \mathrm{~Hz}, \mathrm{C} 4-\mathrm{H}_{2}\right), 2.91\left(\mathrm{t}, 2 \mathrm{H}, J=7.1 \mathrm{~Hz}, \mathrm{C} 5-\mathrm{H}_{2}\right)$; ESI-MS $(+) \mathrm{m} / z$ $211(\mathrm{M}+\mathrm{Na})^{+}$.<smiles>C#CC(=O)c1cccc(C(F)(F)F)c1</smiles>

1-(3-Trifluoromethylphenyl)-2-propyn-1-one. It was synthesized by method A: IR (thin film) 3259, 2101, 1663, 1333, 1223, $1130 \mathrm{~cm}^{-1}$; ${ }^{1} \mathrm{H}$ NMR $\left(500 \mathrm{MHz}, \mathrm{CDCl}_{3}\right) \delta 8.42(\mathrm{~s}, 1 \mathrm{H}, \mathrm{C} 5-\mathrm{H}), 8.36(\mathrm{~d}, 1 \mathrm{H}, J=$ $7.8 \mathrm{~Hz}, \mathrm{C} 9-\mathrm{H}), 7.90$ (d, 1H, J = 7.3 Hz, C7-H), 7.67 (t, 1H, J = 7.8 Hz, C8-H), 3.54 (s, 1H, C3-H); ESI$\mathrm{MS}(+) m / z 199(\mathrm{M}+\mathrm{H})^{+}, 240\left(\mathrm{M}+\mathrm{CH}_{3} \mathrm{CN}+\mathrm{H}\right)^{+}$.<smiles>C#CC(=O)C1CCCCC1</smiles>

1-Cyclohexyl-2-propyn-1-one. It was synthesized by method A: IR (thin film) 3256, 2935, 2857, 2091, 1676, $1224 \mathrm{~cm}^{-1}$; ${ }^{1} \mathrm{H}$ NMR $\left(500 \mathrm{MHz}, \mathrm{CDCl}_{3}\right) \delta 3.22$ (s, 1H, C3-H), $2.43(\mathrm{tt}, 1 \mathrm{H}, J=11.2,3.4 \mathrm{~Hz}, \mathrm{C} 4-\mathrm{H})$, 2.01-1.97 (m, 2H, C5-H, C9-H), 1.82-1.77 (m, 2H, C5-H, C9-H), 1.69-1.64 (m, 1H, C7-H), 1.48-1.40 (m, 2H, C6-H, C8-H), 1.36-1.26 (m, 2H, C6-H, C8-H), 1.24-1.18 (m, 1H, C7-H); ESI-MS (+) m/z 178 $\left(\mathrm{M}+\mathrm{CH}_{3} \mathrm{CN}+\mathrm{H}\right)^{+}$.

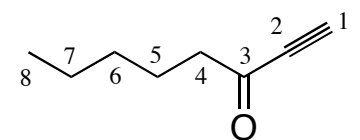

1-Octyn-3-one. It was synthesized by method A: IR (thin film) 3259, 2959, 2934, 2862, 2094, $1685 \mathrm{~cm}^{-1}$; ${ }^{1} \mathrm{H}$ NMR $\left(500 \mathrm{MHz}, \mathrm{CDCl}_{3}\right) \delta 3.21(\mathrm{~s}, 1 \mathrm{H}, \mathrm{C} 1-\mathrm{H}), 2.59\left(\mathrm{t}, 2 \mathrm{H}, J=7.3 \mathrm{~Hz}, \mathrm{C}_{-}-\mathrm{H}_{2}\right), 1.69$ (quint, $2 \mathrm{H}, J=$ $\left.7.3 \mathrm{~Hz}, \mathrm{C} 5-\mathrm{H}_{2}\right), 1.36-1.27\left(\mathrm{~m}, 4 \mathrm{H}, \mathrm{C} 6-\mathrm{H}_{2}, \mathrm{C} 7-\mathrm{H}_{2}\right), 0.90\left(\mathrm{t}, 3 \mathrm{H}, J=7.1 \mathrm{~Hz}, \mathrm{C} 8-\mathrm{H}_{3}\right)$; ESI-MS (+) $m / z 166$ $\left(\mathrm{M}+\mathrm{CH}_{3} \mathrm{CN}+\mathrm{H}\right)^{+}$. 


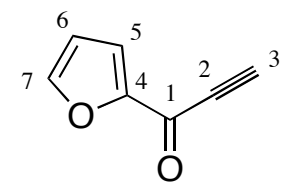

1-(2-Furyl)-2-propyn-1-one. It was synthesized by method A: IR (thin film) 3253, 3147, 2101, 1636, 1459, $1056 \mathrm{~cm}^{-1} ;{ }^{1} \mathrm{H}$ NMR $\left(500 \mathrm{MHz}, \mathrm{CDCl}_{3}\right) \delta 7.70(\mathrm{dd}, 1 \mathrm{H}, J=1.5,1.0 \mathrm{~Hz}, \mathrm{C} 7-\mathrm{H}), 7.42(\mathrm{dd}, 1 \mathrm{H}, J=$ 3.4, $1.0 \mathrm{~Hz}, \mathrm{C} 5-\mathrm{H}), 6.61$ (dd, 1H, $J=3.4,1.5 \mathrm{~Hz}, \mathrm{C} 6-\mathrm{H}), 3.32(\mathrm{~s}, 1 \mathrm{H}, \mathrm{C} 3-\mathrm{H})$; ESI-MS (+) m/z $121(\mathrm{M}+\mathrm{H})$ ,$+ 162\left(\mathrm{M}+\mathrm{CH}_{3} \mathrm{CN}+\mathrm{H}\right)^{+}$.

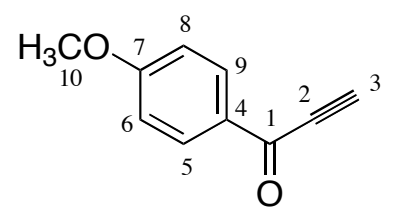

1-(4-Methoxyphenyl)-2-propyn-1-one. It was synthesized by method A: IR (thin film) 3267, 3251, 2097, 1640, 1599, $1260 \mathrm{~cm}^{-1} ;{ }^{1} \mathrm{H}$ NMR (500 MHz, $\mathrm{CDCl}_{3}$ ) $\delta 8.15$ (d, 2H, J = 8.8 Hz, C5-H, C9-H), 6.98 $(\mathrm{d}, 2 \mathrm{H}, J=8.8 \mathrm{~Hz}, \mathrm{C} 6-\mathrm{H}, \mathrm{C} 8-\mathrm{H}), 3.91\left(\mathrm{~s}, 3 \mathrm{H}, \mathrm{C} 10-\mathrm{H}_{3}\right), 3.38(\mathrm{~s}, 1 \mathrm{H}, \mathrm{C} 3-\mathrm{H})$; ESI-MS (+) m/z $161(\mathrm{M}+\mathrm{H})^{+}$, $202\left(\mathrm{M}+\mathrm{CH}_{3} \mathrm{CN}+\mathrm{H}\right)^{+}$.

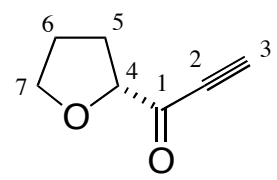

(R)-1-(2-Tetrahydrofuryl)-2-propyn-1-one. It was synthesized by method B: IR (thin film) 3232, 2982, 2881, 2090, 1685, $1061 \mathrm{~cm}^{-1}$; ${ }^{1} \mathrm{H}$ NMR $\left(500 \mathrm{MHz} \mathrm{CDCl}_{3}\right) \delta 4.48$ (dd, 1H, J = 8.3, $\left.5.9 \mathrm{~Hz}, \mathrm{C} 4-\mathrm{H}\right), 4.04$ (q, $1 \mathrm{H}, J=7.3 \mathrm{~Hz}, \mathrm{C} 7-\mathrm{H}), 3.99$ (q, 1H, $J=7.2 \mathrm{~Hz}, \mathrm{C} 7-\mathrm{H}), 3.36$ (s, 1H, C3-H), 2.29-2.24 (m, 1H, C5-H), 2.12 (sextet, $1 \mathrm{H}, J=6.5 \mathrm{~Hz}, \mathrm{C} 5-\mathrm{H}), 1.98-1.92\left(\mathrm{~m}, 2 \mathrm{H}, \mathrm{C} 6-\mathrm{H}_{2}\right)$; ESI-MS (+) $\mathrm{m} / \mathrm{z} 125(\mathrm{M}+\mathrm{H})^{+}$.

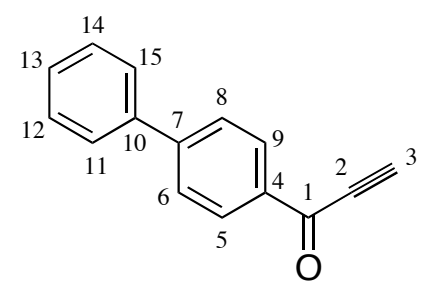

1-(4-Biphhenyl)-2-propyn-1-one. It was synthesized by method A: IR (thin film) 3238, 2094, 1685, 1061, $740 \mathrm{~cm}^{-1}$; ${ }^{1} \mathrm{H}$ NMR $\left(500 \mathrm{MHz}, \mathrm{CDCl}_{3}\right) \delta 8.25(\mathrm{~d}, 2 \mathrm{H}, J=8.3 \mathrm{~Hz}, \mathrm{C} 5-\mathrm{H}, \mathrm{C} 9-\mathrm{H}), 7.74(\mathrm{~d}, 2 \mathrm{H}, J=8.3$ Hz, C6-H, C8-H), 7.65 (d, 2H, J = 7.3 Hz, C11-H, C15-H), 7.50 (t, 2H, J = 7.6 Hz, C12-H, C14-H), 7.43 $(\mathrm{t}, 1 \mathrm{H}, J=7.3 \mathrm{~Hz}, \mathrm{C} 13-\mathrm{H}), 3.46(\mathrm{~s}, 1 \mathrm{H}, \mathrm{C} 3-\mathrm{H})$; ESI-MS $(+) \mathrm{m} / z 207(\mathrm{M}+\mathrm{H})^{+}, 248\left(\mathrm{M}+\mathrm{CH}_{3} \mathrm{CN}+\mathrm{H}\right)^{+}$.

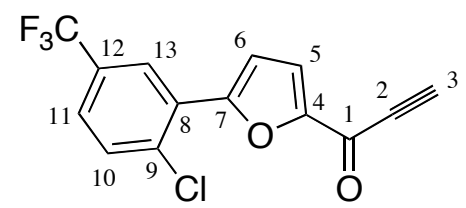

1-[5-(2-Chloro-5-trifluoromethylphenyl)furan-2-yl]-2-propyn-1-one. It was synthesized by method B: IR (thin film) 3238, 2100, 1636, 1328, 1131, $746 \mathrm{~cm}^{-1} ;{ }^{1} \mathrm{H}$ NMR $\left(500 \mathrm{MHz}, \mathrm{CDCl}_{3}\right) \delta 8.27$ (d, $1 \mathrm{H}, J=2.0$ $\mathrm{Hz}, \mathrm{C} 13-\mathrm{H}), 7.63(\mathrm{~d}, 1 \mathrm{H}, J=8.3 \mathrm{~Hz}, \mathrm{C} 10-\mathrm{H}), 7.58(\mathrm{dd}, 1 \mathrm{H}, J=8.3,2.0 \mathrm{~Hz}, \mathrm{C} 11-\mathrm{H}), 7.53(\mathrm{~d}, 1 \mathrm{H}, J=3.9$ $\mathrm{Hz}, \mathrm{C} 5-\mathrm{H}), 7.38$ (d, 1H, J = 3.4 Hz, C6-H), 3.39 (s, 1H, C3-H); ESI-MS (+) $m / z .299,301(\mathrm{M}+\mathrm{H})^{+}, 340$, $342\left(\mathrm{M}+\mathrm{CH}_{3} \mathrm{CN}+\mathrm{H}\right)^{+}$. 


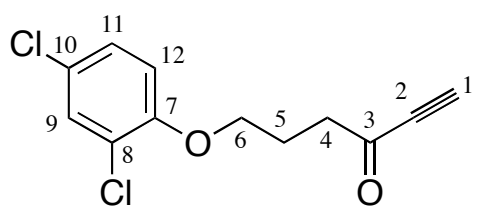

6-(2,4-Dichlorophenoxy)-1-hexyn-3-one. It was synthesized by method A: IR (thin film) 3287, 3075, 2960, 2886, 2096, 1685, 1486, 1290, $1063 \mathrm{~cm}^{-1}$; ${ }^{1} \mathrm{H}$ NMR $\left(500 \mathrm{MHz}, \mathrm{CDCl}_{3}\right) \delta 7.36(\mathrm{~d}, 1 \mathrm{H}, J=2.4 \mathrm{~Hz}$, C9-H), $7.16(\mathrm{dd}, 1 \mathrm{H}, J=8.8,2.9 \mathrm{~Hz}, \mathrm{C} 11-\mathrm{H}), 6.82(\mathrm{~d}, 1 \mathrm{H}, J=8.8 \mathrm{~Hz}, \mathrm{C} 12-\mathrm{H}), 4.03(\mathrm{t}, 2 \mathrm{H}, J=6.1 \mathrm{~Hz}$, C6- $\mathrm{H}_{2}$ ), 3.26 (s, 1H, C1-H), 2.89 (t, 2H, $J=6.8 \mathrm{~Hz}, \mathrm{C} 4-\mathrm{H}_{2}$ ), 2.19 (sept, 2H, $J=6.5 \mathrm{~Hz}, \mathrm{C} 5-\mathrm{H}_{2}$ ); ESI-MS (+) $m / z, 257,259,261(\mathrm{M}+\mathrm{H})^{+}$.<smiles>C#CC(=O)Cc1c(F)cccc1Cl</smiles>

1-(2-Chloro-6-fluorophenyl)-3-butyn-2-one. It was synthesized by method A: IR (thin film) 3289, 2097 , 1686, 1468, $942 \mathrm{~cm}^{-1}$; ${ }^{1} \mathrm{H}$ NMR $\left(500 \mathrm{MHz}^{\mathrm{CDCl}}{ }_{3}\right) \delta$ 7.26-7.23 (m, 2H, C7-H, C8-H), 7.06-7.02 (m, 1H, C9-H), $4.10\left(\mathrm{~d}, 2 \mathrm{H}, J=2.0 \mathrm{~Hz}, \mathrm{C} 1-\mathrm{H}_{2}\right), 3.26(\mathrm{~s}, 1 \mathrm{H}, \mathrm{C} 4-\mathrm{H})$; ESI-MS (+) $m / z 238\left(\mathrm{M}+\mathrm{CH}_{3} \mathrm{CN}+\mathrm{H}\right)^{+}$.<smiles>C#CC(=O)COC1CC(C)CCC1C(C)C</smiles>

1-[(1R)-Menthyloxy]-3-butyn-2-one. It was synthesized by method B: IR (thin film) 3288, 2956, 2923, 2871, 2096, 1685, 1065, $748 \mathrm{~cm}^{-1} ;{ }^{1} \mathrm{H}$ NMR $\left(500 \mathrm{MHz}, \mathrm{CDCl}_{3}\right) \delta 4.31$ (d, 1H, J = 17.6 Hz, C1-H), 4.22 $(\mathrm{d}, 1 \mathrm{H}, J=18.1 \mathrm{~Hz}, \mathrm{C} 1-\mathrm{H}), 3.31(\mathrm{~s}, 1 \mathrm{H}, \mathrm{C} 4-\mathrm{H}), 3.18(\mathrm{td}, 1 \mathrm{H}, J=12.0,3.9 \mathrm{~Hz}, \mathrm{C} 5-\mathrm{H}), 2.37-2.29(\mathrm{~m}, 1 \mathrm{H}$, C11-H), 2.06-2.04 (m, 1H, C10-H), 1.67-1.65 (m, 2H, C7-H, C8-H), 1.36-1.32 (m, 2H, C9-H, C6-H), $1.00-0.93(\mathrm{~m}, 2 \mathrm{H}, \mathrm{C} 7-\mathrm{H}, \mathrm{C} 10-\mathrm{H}), 0.94\left(\mathrm{~d}, 3 \mathrm{H}, J=6.3 \mathrm{~Hz}, \mathrm{C} 14-\mathrm{H}_{3}\right), 0.93$ (d, 3H, $J=6.8 \mathrm{~Hz}, \mathrm{C} 12-\mathrm{H}_{3}$ or C13- $\left.\mathrm{H}_{3}\right), 0.91-0.85(\mathrm{~m}, 1 \mathrm{H}, \mathrm{C} 8-\mathrm{H}), 0.81\left(\mathrm{~d}, 3 \mathrm{H}, \mathrm{J}=6.8 \mathrm{~Hz}, \mathrm{C} 12-\mathrm{H}_{3}\right.$ or C13-H $)$; ESI-MS (+) $m / z 223$ $(\mathrm{M}+\mathrm{H})^{+}$.

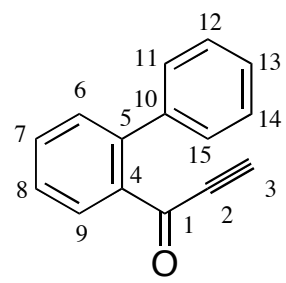

1-(2-Biphhenyl)-2-propyn-1-one. It was synthesized by method B: IR (thin film) 3281, 3061, 2956, 2095, 1660, $747 \mathrm{~cm}^{-1}$; ${ }_{1}^{1} \mathrm{H}$ NMR $\left(500 \mathrm{MHz}, \mathrm{CDCl}_{3}\right) \delta 8.00(\mathrm{~d}, 1 \mathrm{H}, J=7.8 \mathrm{~Hz}, \mathrm{C} 9-\mathrm{H}), 7.61(\mathrm{t}, 1 \mathrm{H}, J=7.6$ $\mathrm{Hz}, \mathrm{C} 7-\mathrm{H}), 7.49$ (t, 1H, J = 7.6 Hz, C8-H), 7.43-7.38 (m, 4H, C11-H, C12-H, C14-H, C15-H), 7.34-7.32 (m, 2H, C6-H, C13-H), 3.07 (s, 1H, C3-H); ESI-MS (+) m/z $207(\mathrm{M}+\mathrm{H})^{+}$.

\section{5) Structure confirmation of library members}

The scheme below shows the structures and synthesis route for fully characterized library members. These compounds were synthesized by the optimized reaction conditions described above, then cleaved from the solid support. 

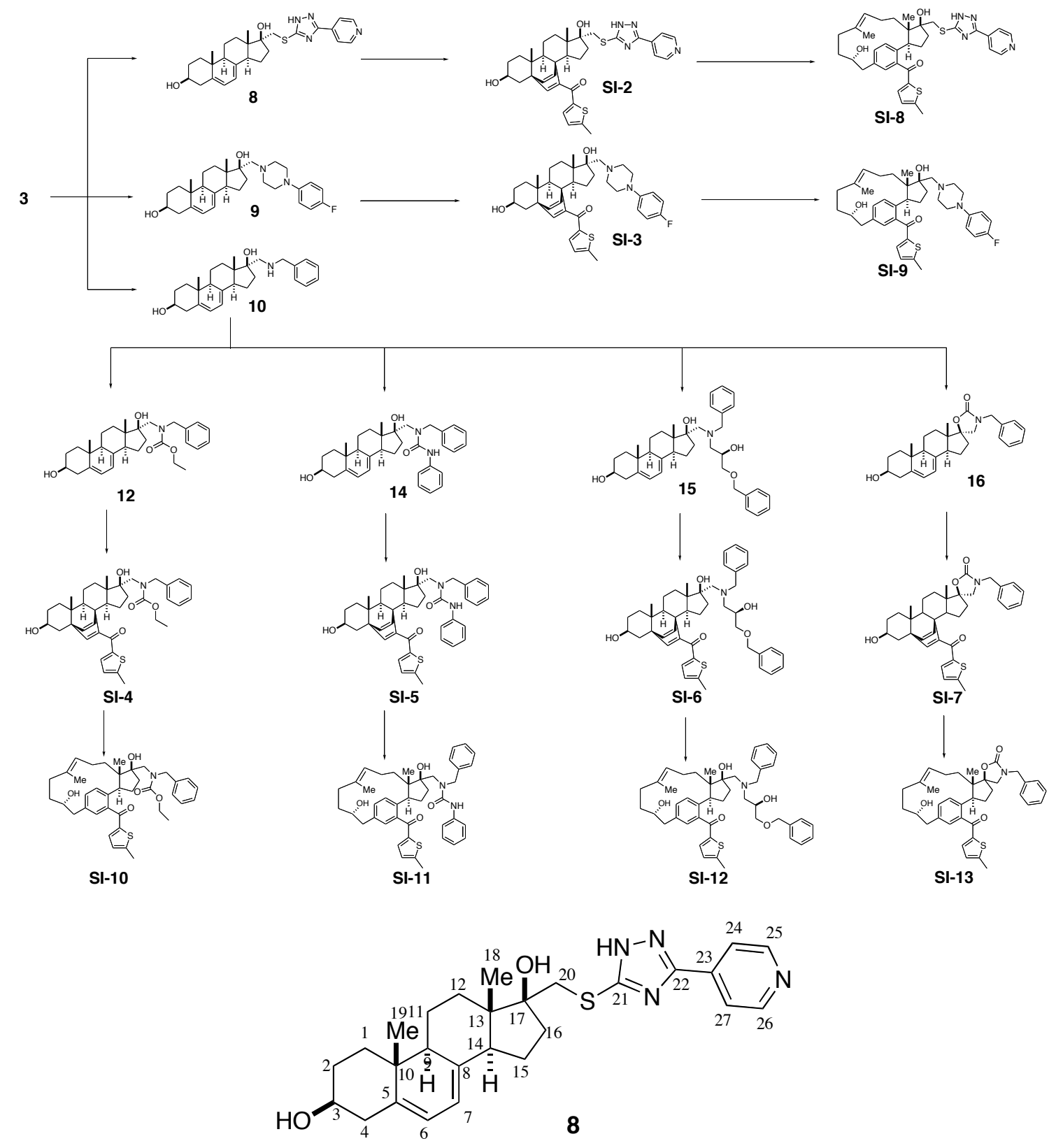

17a-[5-(4-Pyridyl)-1 $H$-[1,2,4]triazol-3-yl]thiomethylandrosta-5,7-dien-3b,17b-diol (8): mp 210-211 ${ }^{\circ} \mathrm{C}$; IR (thin film) 3449, 2982, 2870, $1073 \mathrm{~cm}^{-1}$; ${ }^{1} \mathrm{H}$ NMR (500 MHz, DMSO-d 6 ) $\delta 14.25$ (br.s, $1 \mathrm{H}, \mathrm{NH}$ ), 8.67 (br.s, $2 \mathrm{H}, \mathrm{C} 25-\mathrm{H}, \mathrm{C} 26-\mathrm{H}$ ), 7.87 (d, 2H, $J=5.4 \mathrm{~Hz}, \mathrm{C} 24-\mathrm{H}, \mathrm{C} 27-\mathrm{H}), 5.48$ (dd, $1 \mathrm{H}, J=5.3,2.0 \mathrm{~Hz}$, C6-H), 5.34 (dt, 1H, $J=5.4,2.6 \mathrm{~Hz}, \mathrm{C} 7-\mathrm{H}), 4.80$ (br.s, $1 \mathrm{H}, \mathrm{C} 17-\mathrm{OH}), 4.69$ (d, $1 \mathrm{H}, J=3.9 \mathrm{~Hz}, \mathrm{C} 3-\mathrm{OH})$, 3.55-3.45 (m, 2H, C20- $\mathrm{H}_{2}$ ), 3.38-3.33 (m, 1H, C3-H), 2.31 (ddd, 1H, $\left.J=14.6,4.2,2.2 \mathrm{~Hz}, \mathrm{C} 4-\mathrm{H}\right), 2.18$ (t, $1 \mathrm{H}, J=8.3 \mathrm{~Hz}, \mathrm{C} 14-\mathrm{H}), 2.16-2.07(\mathrm{~m}, 2 \mathrm{H}, \mathrm{C} 4-\mathrm{H}, \mathrm{C} 16-\mathrm{H}), 1.90$ (t, $1 \mathrm{H}, J=8.8 \mathrm{~Hz}, \mathrm{C} 9-\mathrm{H}), 1.80$ (dt, $1 \mathrm{H}, J=13.2,3.4 \mathrm{~Hz}, \mathrm{C} 1-\mathrm{H}), 1.74-1.65$ (m, 3H, C2-H, C12-H, C16-H), 1.66-1.59 (m, 3H, C15-H, C11H2), 1,47 (qd, 1H, $J=11.7,6.8 \mathrm{~Hz}, \mathrm{C} 15-\mathrm{H}), 1.43-1.36(\mathrm{~m}, 1 \mathrm{H}, \mathrm{C} 12-\mathrm{H}), 1.33$ (qd, $1 \mathrm{H}, J=12.0,2.9 \mathrm{~Hz}$, $\mathrm{C} 2-\mathrm{H}), 1.20(\mathrm{td}, 1 \mathrm{H}, J=13.7,3.4 \mathrm{~Hz}, \mathrm{C} 1-\mathrm{H}), 0.86\left(\mathrm{~s}, 3 \mathrm{H}, \mathrm{C} 19-\mathrm{H}_{3}\right), 0.73\left(\mathrm{~s}, 3 \mathrm{H}, \mathrm{C} 18-\mathrm{H}_{3}\right) ;{ }^{13} \mathrm{C} \mathrm{NMR}(125$ $\left.\mathrm{MHz}, \mathrm{DMSO}-d_{6}\right) \delta 159.32(\mathrm{C}, \mathrm{C} 23), 154.80$ (C, C21), 150.36 (CH, C25, C26), 140.61 (C, C8), 140.47 (C, C5), 137.93 (C, C22), 119.91 (CH, C24, C27), 118.63 (CH, C6), 116.07 (CH, C7), 82.10 (C, C17), $68.53(\mathrm{CH}, \mathrm{C} 3), 48.51(\mathrm{CH}, \mathrm{C} 14), 46.77$ (C, C13), $45.78(\mathrm{CH}, \mathrm{C} 9), 42.59\left(\mathrm{CH}_{2}, \mathrm{C} 20\right), 40.69\left(\mathrm{CH}_{2}, \mathrm{C} 4\right)$, $37.90\left(\mathrm{CH}_{2}, \mathrm{C} 1\right), 36.62(\mathrm{C}, \mathrm{C} 10), 34.03\left(\mathrm{CH}_{2}, \mathrm{C} 16\right), 31.76\left(\mathrm{CH}_{2}, \mathrm{C} 2\right), 30.77\left(\mathrm{CH}_{2}, \mathrm{C} 12\right), 22.21\left(\mathrm{CH}_{2}\right.$, C15), $20.35\left(\mathrm{CH}_{2}, \mathrm{C} 11\right), 16.07\left(\mathrm{CH}_{3}, \mathrm{C} 19\right), 14.71\left(\mathrm{CH}_{3}, \mathrm{C} 18\right)$; ESI-MS $(+) \mathrm{m} / z .779(\mathrm{M}+\mathrm{H})^{+}$. 


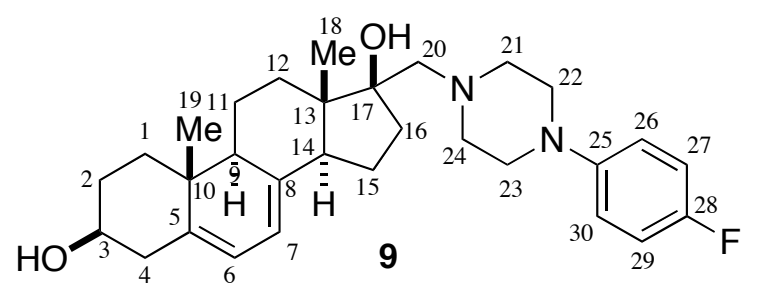

17a-[4-(4-Fluorophenyl)piperazin-1-ylmethyl]androsta-5,7-dien-3b,17b-diol (9):

mp 189-190 ${ }^{\circ} \mathrm{C}$; IR (thin film) 3405, 2962, 2930, 1510, 1275, $748 \mathrm{~cm}^{-1} ;{ }^{1} \mathrm{H}$ NMR (500 MHz, $\left.\mathrm{CDCl}_{3}\right) \delta$ $6.95(\mathrm{t}, 2 \mathrm{H}, J=8.6 \mathrm{~Hz}, \mathrm{C} 27-\mathrm{H}, \mathrm{C} 29-\mathrm{H}), 6.86(\mathrm{dd}, 2 \mathrm{H}, J=9.0,4.7 \mathrm{~Hz}, \mathrm{C} 26-\mathrm{H}, \mathrm{C} 30-\mathrm{H}), 5.57$ (dd, $1 \mathrm{H}, J=$ 5.4, 2.4 Hz, C6-H), $5.42(\mathrm{dt}, 1 \mathrm{H}, J=5.9,2.4 \mathrm{~Hz}, \mathrm{C} 7-\mathrm{H}), 3.63(\mathrm{tt}, 1 \mathrm{H}, J=11.2,4.4 \mathrm{~Hz}, \mathrm{C} 3-\mathrm{H}), 3.12(\mathrm{t}, 4 \mathrm{H}$, $\left.J=4.9 \mathrm{~Hz}, \mathrm{C} 22-\mathrm{H}_{2}, \mathrm{C} 23-\mathrm{H}_{2}\right), 2.86(\mathrm{dt}, 2 \mathrm{H}, J=11.2,4.9 \mathrm{~Hz}, \mathrm{C} 21-\mathrm{H}, \mathrm{C} 24-\mathrm{H}), 2.79$ (dt, $2 \mathrm{H}, J=11.4,4.9$ Hz, C21-H, C24-H), 2.70 (d, 1H, $J=13.2 \mathrm{~Hz}, \mathrm{C} 20-\mathrm{H}), 2.47$ (ddd, 1H, $J=14.4,4.7,2.4 \mathrm{~Hz}, \mathrm{C} 4-\mathrm{H}), 2.43$ $(\mathrm{d}, 1 \mathrm{H}, J=13.2 \mathrm{~Hz}, \mathrm{C} 20-\mathrm{H}), 2.28(\mathrm{td}, 1 \mathrm{H}, J=12.9,1.5 \mathrm{~Hz}, \mathrm{C} 4-\mathrm{H}), 2.08(\mathrm{ddd}, 1 \mathrm{H}, J=14.2,9.6,6.8 \mathrm{~Hz}$, C16-H), 2.01 (td, 1H, J = 9.3, $1.5 \mathrm{~Hz}, \mathrm{C} 14-\mathrm{H}), 1.95$ (t, 1H, $J=8.8 \mathrm{~Hz}, \mathrm{C} 9-\mathrm{H}), 1.92-1.86$ (m, 2H, C1-H, $\mathrm{C} 2-\mathrm{H}), 1.83(\mathrm{td}, 1 \mathrm{H}, J=13.0,3.1 \mathrm{~Hz}, \mathrm{C} 16-\mathrm{H}), 1.77-1.70(\mathrm{~m}, 2 \mathrm{H}, \mathrm{C} 11-\mathrm{H}, \mathrm{C} 15-\mathrm{H}), 1.69-1.60(\mathrm{~m}, 3 \mathrm{H}$, C11-H, C12-H, C15-H), 1.50 (dtd, 1H, J = 13.3, 11.2, $4.2 \mathrm{~Hz}, \mathrm{C} 2-\mathrm{H}), 1.34$ (td, 1H, J = 12.7, 4.9 Hz, C12$\mathrm{H}), 1.29(\mathrm{td}, 1 \mathrm{H}, J=13.7,3.9 \mathrm{~Hz}, \mathrm{C} 1-\mathrm{H}), 0.96\left(\mathrm{~s}, 3 \mathrm{H}, \mathrm{C} 19-\mathrm{H}_{3}\right), 0.85\left(\mathrm{~s}, 3 \mathrm{H}, \mathrm{C} 18-\mathrm{H}_{3}\right) ;{ }^{13} \mathrm{C} \mathrm{NMR}(125$

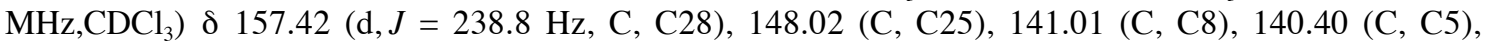
119.66 (CH, C6), 117.99 (d, $J=7.6 \mathrm{~Hz}, \mathrm{CH}, \mathrm{C} 26, \mathrm{C} 30), 117.01(\mathrm{CH}, \mathrm{C} 7), 115.72$ (d, J = 22.1 Hz, CH, C27, C29), 81.88 (C, C17), $70.54(\mathrm{CH}, \mathrm{C} 3), 63.51\left(\mathrm{CH}_{2}, \mathrm{C} 20\right), 55.55\left(\mathrm{CH}_{2}, \mathrm{C} 121, \mathrm{C} 24\right), 50.67\left(\mathrm{CH}_{2}\right.$, C22, C23), 49.06 (CH, C14), 46.62 (CH, C9), 45.96 (C, C13), $40.97\left(\mathrm{CH}_{2}, \mathrm{C} 4\right), 38.55\left(\mathrm{CH}_{2}, \mathrm{C} 1\right), 37.35$ (C, C10), $36.64\left(\mathrm{CH}_{2}, \mathrm{C} 16\right), 32.16\left(\mathrm{CH}_{2}, \mathrm{C} 2\right), 31.70\left(\mathrm{CH}_{2}, \mathrm{C} 12\right), 22.87\left(\mathrm{CH}_{2}, \mathrm{C} 15\right), 20.95\left(\mathrm{CH}_{2}, \mathrm{C} 11\right)$, $16.50\left(\mathrm{CH}_{3}, \mathrm{C} 19\right), 15.04\left(\mathrm{CH}_{3}, \mathrm{C} 18\right)$; ESI-MS $(+) m / z 481(\mathrm{M}+\mathrm{H})^{+}$.

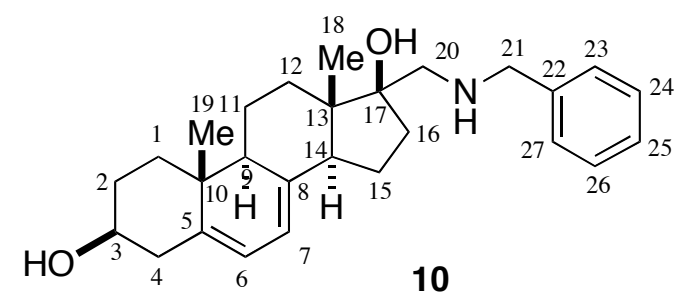

17a-Benzylaminomethylandrosta-5,7-dien-3b,17b-diol (10): $\mathrm{mp} 145{ }^{\circ} \mathrm{C}$; IR (thin film) 3278,3036 , 2962, 2931, 2854, 1453, 1064, 732, $698 \mathrm{~cm}^{-1}$; ${ }^{1} \mathrm{H}$ NMR $\left(500 \mathrm{MHz}, \mathrm{CDCl}_{3}\right) \delta 7.33(\mathrm{t}, 2 \mathrm{H}, J=6.8 \mathrm{~Hz}, \mathrm{C} 24-$ $\mathrm{H}, \mathrm{C} 26-\mathrm{H}), 7.31(\mathrm{~d}, 2 \mathrm{H}, J=6.8 \mathrm{~Hz}, \mathrm{C} 23-\mathrm{H}, \mathrm{C} 27-\mathrm{H}), 7.27(\mathrm{t}, 1 \mathrm{H}, J=6.8 \mathrm{~Hz}, \mathrm{C} 25-\mathrm{H}), 5.55(\mathrm{dd}, 1 \mathrm{H}, J=$ 5.5, $2.2 \mathrm{~Hz}, \mathrm{C} 6-\mathrm{H}), 5.40$ (dt, $1 \mathrm{H}, J=5.5,2.5 \mathrm{~Hz}, \mathrm{C} 7-\mathrm{H}), 3.84\left(\mathrm{~s}, 2 \mathrm{H}, \mathrm{C} 21-\mathrm{H}_{2}\right), 3.63(\mathrm{tt}, 1 \mathrm{H}, J=11.2,4.4$ $\mathrm{Hz}, \mathrm{C} 3-\mathrm{H}), 2.88(\mathrm{~d}, 1 \mathrm{H}, J=11.7 \mathrm{~Hz}, \mathrm{C} 20-\mathrm{H}), 2.54(\mathrm{~d}, 1 \mathrm{H}, J=11.7 \mathrm{~Hz}, \mathrm{C} 20-\mathrm{H}), 2.46$ (ddd, 1H, $J=14.4$, 4.6, $2.0 \mathrm{~Hz}, \mathrm{C} 4-\mathrm{H}), 2.28$ (t, 1H, $J=12.2 \mathrm{~Hz}, \mathrm{C} 4-\mathrm{H}), 2.04(\mathrm{td}, 1 \mathrm{H}, J=9.3,2.0 \mathrm{~Hz}, \mathrm{C} 14-\mathrm{H}), 1.94(\mathrm{t}, 1 \mathrm{H}, J=$ $8.3 \mathrm{~Hz}, \mathrm{C} 9-\mathrm{H}), 1.91-1.75$ (m, 4H, C1-H, C2-H, C16-H $)$, 1.73-1.56 (m, 5H, C11-H 2 , C12-H, C15-H $)$, $1.49(\mathrm{dtd}, 1 \mathrm{H}, J=13.3,11.0,4.2 \mathrm{~Hz}, \mathrm{C} 2-\mathrm{H}), 1.27(\mathrm{td}, 1 \mathrm{H}, J=13.7,3.9 \mathrm{~Hz}, \mathrm{C} 1-\mathrm{H}), 1.23(\mathrm{td}, 1 \mathrm{H}, J=12.7$, $4.9 \mathrm{~Hz}, \mathrm{C} 12-\mathrm{H}), 0.95$ (s, 3H, C19-H $), 0.83\left(\mathrm{~s}, 3 \mathrm{H}, \mathrm{C} 18-\mathrm{H}_{3}\right) ;{ }^{13} \mathrm{C} \mathrm{NMR}\left(125 \mathrm{MHz}, \mathrm{CDCl}_{3}\right) \delta 140.76(\mathrm{C}$, C8), 140.07 (C, C5, C22), 128.51 (CH, C24, C26), $128.03(\mathrm{CH}, \mathrm{C} 23, \mathrm{C} 27), 127.18(\mathrm{CH}, \mathrm{C} 25), 119.42$ (CH, C6), $116.64(\mathrm{CH}, \mathrm{C} 7), 81.80(\mathrm{C}, \mathrm{C} 17), 70.32(\mathrm{CH}, \mathrm{C} 3), 55.48\left(\mathrm{CH}_{2}, \mathrm{C} 20\right), 54.54\left(\mathrm{CH}_{2}, \mathrm{C} 21\right), 48.93$ (CH, C14), 46.28 (CH, C9), 45.46 (C, C13), $40.73\left(\mathrm{CH}_{2}, \mathrm{C} 4\right), 38.31\left(\mathrm{CH}_{2}, \mathrm{C} 1\right), 37.09(\mathrm{C}, \mathrm{C} 10), 35.43$ $\left(\mathrm{CH}_{2}, \mathrm{C} 16\right), 32.14\left(\mathrm{CH}_{2}, \mathrm{C} 12\right), 31.92\left(\mathrm{CH}_{2}, \mathrm{C} 2\right), 22.39\left(\mathrm{CH}_{2}, \mathrm{C} 15\right), 20.69\left(\mathrm{CH}_{2}, \mathrm{C} 11\right), 16.30\left(\mathrm{CH}_{3}, \mathrm{C} 19\right)$, $14.58\left(\mathrm{CH}_{3}, \mathrm{C} 18\right)$; ESI-MS (+) $m / z, 408(\mathrm{M}+\mathrm{H})^{+}$.<smiles>[Y19]C12CCC3C(=CC=C4CC(O)CCC43C)[C@@]1(C)CCC2(O)CN(Cc1ccccc1)C(=O)OCC</smiles>

$N$-Benzyl- $N$-(3b,17b-dihydroxyandrosta-5,7-dien-17a-ylmethyl)carbamic acid ethyl ester (12): IR 
(thin film) 3398, 2972, 2931, 1672, $729 \mathrm{~cm}^{-1} ;{ }^{1} \mathrm{H}$ NMR $\left(500 \mathrm{MHz}, \mathrm{CDCl}_{3}\right) \delta 7.33$ (t, 2H, $J=7.1 \mathrm{~Hz}, \mathrm{C} 24-$ H, C26-H), 7.27 (t, $1 \mathrm{H}, J=6.8 \mathrm{~Hz}, \mathrm{C} 25-\mathrm{H}), 7.19$ (d, 2H, $J=5.9 \mathrm{~Hz}, \mathrm{C} 23-\mathrm{H}, \mathrm{C} 27-\mathrm{H}), 5.57$ (br.s, $1 \mathrm{H}, \mathrm{C} 6-$ H), 5.41 (br.s, 1H, C7-H), 4.87 (d, 1H, $J=15.1 \mathrm{~Hz}, \mathrm{C} 21-\mathrm{H}), 4.35$ (d, 1H, $J=15.1 \mathrm{~Hz}, \mathrm{C} 21-\mathrm{H}), 4.20$ (br.s, $\left.2 \mathrm{H}, \mathrm{C} 29-\mathrm{H}_{2}\right), 3.63(\mathrm{tt}, 1 \mathrm{H}, J=11.0,3.9 \mathrm{~Hz}, \mathrm{C} 3-\mathrm{H}), 3.51(\mathrm{~d}, 1 \mathrm{H}, J=14.7 \mathrm{~Hz}, \mathrm{C} 20-\mathrm{H}), 3.30(\mathrm{~d}, 1 \mathrm{H}, J=$ $14.7 \mathrm{~Hz}, \mathrm{C} 20-\mathrm{H}), 2.49-2.46$ (m, 1H, C4-H), 2.28 (t, 1H, J = 12.7 Hz, C4-H), 2.15-2.04 (m, 2H, C14-H, C16-H), 1.95-1.84 (m, 3H, C1-H, C2-H, C9-H), 1.74-1.56 (m, 6H, C11- $\left.{ }_{2}, \mathrm{C} 12-\mathrm{H}, \mathrm{C} 15-\mathrm{H}_{2}, \mathrm{C} 16-\mathrm{H}\right)$, 1.49 (qd, $1 \mathrm{H}, J=11.2,4.2 \mathrm{~Hz}, \mathrm{C} 2-\mathrm{H}), 1.29-1.22(\mathrm{~m}, 2 \mathrm{H}, \mathrm{C} 1-\mathrm{H}, \mathrm{C} 12-\mathrm{H}), 1.26$ (t, $3 \mathrm{H}, J=6.8 \mathrm{~Hz}, \mathrm{C} 30-\mathrm{H}_{3}$ ), $0.95\left(\mathrm{~s}, 3 \mathrm{H}, \mathrm{C} 19-\mathrm{H}_{3}\right), 0.82\left(\mathrm{~s}, 3 \mathrm{H}, \mathrm{C} 18-\mathrm{H}_{3}\right) ;{ }^{13} \mathrm{C} \mathrm{NMR}\left(125 \mathrm{MHz}, \mathrm{CDCl}_{3}\right) \delta 159.42(\mathrm{C}, \mathrm{C} 28), 140.97(\mathrm{C}$, C8), 140.38 (C, C5), 137.85 (C, C22), 128.84 (CH, C24, C26), 127.44 (CH, C23, C25, C27), 119.62 (CH, C6), $116.90(\mathrm{CH}, \mathrm{C} 7), 85.12(\mathrm{C}, \mathrm{C} 17), 70.54(\mathrm{CH}, \mathrm{C} 3), 62.40\left(\mathrm{CH}_{2}, \mathrm{C} 29\right), 54.18\left(\mathrm{CH}_{2}, \mathrm{C} 20\right), 53.32\left(\mathrm{CH}_{2}\right.$, C21), 48.80 (CH, C14), 46.57 (CH, C9), 46.47 (C, C13), $40.97\left(\mathrm{CH}_{2}, \mathrm{C} 4\right), 38.52\left(\mathrm{CH}_{2}, \mathrm{C} 1\right), 37.36(\mathrm{C}$, $\mathrm{C} 10), 33.04\left(\mathrm{CH}_{2}, \mathrm{C} 16\right), 32.15\left(\mathrm{CH}_{2}, \mathrm{C} 2\right), 30.36\left(\mathrm{CH}_{2}, \mathrm{C} 12\right), 22.40\left(\mathrm{CH}_{2}, \mathrm{C} 15\right), 20.75\left(\mathrm{CH}_{2}, \mathrm{C} 11\right), 16.51$ $\left(\mathrm{CH}_{3}, \mathrm{C} 19\right), 14.81\left(\mathrm{CH}_{3}, \mathrm{C} 30\right), 14.38\left(\mathrm{CH}_{3}, \mathrm{C} 18\right)$; ESI-MS $(+) \mathrm{m} / z 502(\mathrm{M}+\mathrm{Na})^{+}, 543\left(\mathrm{M}+\mathrm{CH}_{3} \mathrm{CN}+\mathrm{Na}\right)^{+}$.

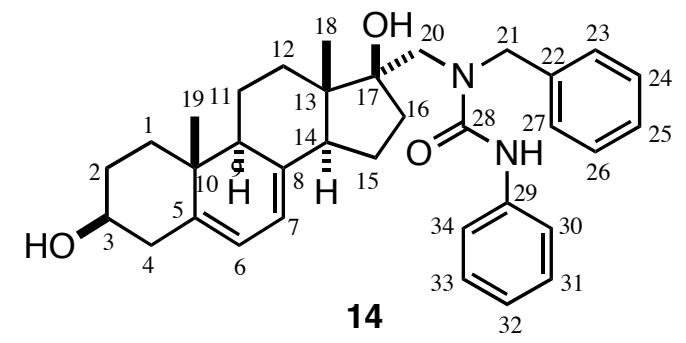

1-Benzyl-1-(3b,17b-dihydroxyandrosta-5,7-dien-17a-ylmethyl)-3-phenylurea (14): $\mathrm{mp}$ 196-197 ${ }^{\circ} \mathrm{C}$; IR (thin film) 3320, 3035, 2963, 2933, 1654, 1405, 1315, 1245, 1038, $732 \mathrm{~cm}^{-1} ;{ }^{1} \mathrm{H}$ NMR $(500 \mathrm{MHz}$, $\left.\mathrm{CDCl}_{3}\right) \delta 7.36(\mathrm{t}, 2 \mathrm{H}, J=7.3 \mathrm{~Hz}, \mathrm{C} 24-\mathrm{H}, \mathrm{C} 26-\mathrm{H}), 7.34-7.28(\mathrm{~m}, 5 \mathrm{H}, \mathrm{C} 23-\mathrm{H}, \mathrm{C} 25-\mathrm{H}, \mathrm{C} 27-\mathrm{H}, \mathrm{C} 30-\mathrm{H}$, C34-H), 7.25 (t, $2 \mathrm{H}, J=7.3 \mathrm{~Hz}, \mathrm{C} 31-\mathrm{H}, \mathrm{C} 33-\mathrm{H}), 6.99(\mathrm{t}, 1 \mathrm{H}, J=7.3 \mathrm{~Hz}, \mathrm{C} 32-\mathrm{H}), 5.59$ (dd, $1 \mathrm{H}, J=5.4$, $2.4 \mathrm{~Hz}, \mathrm{C} 6-\mathrm{H}), 5.43(\mathrm{dt}, 1 \mathrm{H}, J=5.4,2.7 \mathrm{~Hz}, \mathrm{C} 7-\mathrm{H}), 5.18(\mathrm{~d}, 1 \mathrm{H}, J=16.1 \mathrm{~Hz}, \mathrm{C} 21-\mathrm{H}), 4.13(\mathrm{~d}, 1 \mathrm{H}, J=$ $16.1 \mathrm{~Hz}, \mathrm{C} 21-\mathrm{H}), 3.72$ (d, $1 \mathrm{H}, J=15.6 \mathrm{~Hz}, \mathrm{C} 20-\mathrm{H}), 3.64$ (tt, $1 \mathrm{H}, J=11.2,4.2 \mathrm{~Hz}, \mathrm{C} 3-\mathrm{H}), 3.25(\mathrm{~d}, 1 \mathrm{H}, J=$ $15.6 \mathrm{~Hz}, \mathrm{C} 20-\mathrm{H}), 2.49$ (ddd, $1 \mathrm{H}, J=14.2,4.9,2.4 \mathrm{~Hz}, \mathrm{C} 4-\mathrm{H}), 2.37-2.31(\mathrm{~m}, 1 \mathrm{H}, \mathrm{C} 16-\mathrm{H}), 2.29(\mathrm{t}, 1 \mathrm{H}, J=$ $12.2 \mathrm{~Hz}, \mathrm{C} 4-\mathrm{H}), 2.06$ (t, $1 \mathrm{H}, J=8.3 \mathrm{~Hz}, \mathrm{C} 14-\mathrm{H}), 1.94(\mathrm{t}, 1 \mathrm{H}, J=8.3 \mathrm{~Hz}, \mathrm{C} 9-\mathrm{H}), 1.91-1.87$ (m, 2H, C1-H, C2-H), 1.79-1.56 (m, 6H, C11- $\left.\mathrm{H}_{2}, \mathrm{C} 12-\mathrm{H}, \mathrm{C} 15-\mathrm{H}_{2}, \mathrm{C} 16-\mathrm{H}\right), 1.50$ (dtd, $1 \mathrm{H}, J=13.2,11.3,3.4 \mathrm{~Hz}, \mathrm{C} 2-\mathrm{H}$, 1.31-1.25 (m, 3H, C1-H, C12-H, C3-OH), 0.97 (s, 3H, C19- $\left.\mathrm{H}_{3}\right), 0.87$ (s, 3H, C18- $\left.\mathrm{H}_{3}\right) ;{ }^{13} \mathrm{C}$ NMR $(125$ $\mathrm{MHz}, \mathrm{CDCl}_{3}$ ) $\delta 158.11$ (C, C28), 140.71 (C, C5), 140.21 (C, C8), 139.85 (C, C29), 137.63 (C, C22), $129.14(\mathrm{CH}, \mathrm{C} 24, \mathrm{C} 26), 128.98$ (CH, C31, C33), $127.81(\mathrm{CH}, \mathrm{C} 23, \mathrm{C} 27), 127.56(\mathrm{CH}, \mathrm{C} 25), 122.77(\mathrm{CH}$, C32), 119.62 (CH, C30, C34), 119.53 (CH, C6), 117.24 (CH, C7), 86.11 (C, C17), 70.49 (CH, C3), 54.25 $\left(\mathrm{CH}_{2}, \mathrm{C} 20\right), 53.70\left(\mathrm{CH}_{2}, \mathrm{C} 21\right), 48.60(\mathrm{CH}, \mathrm{C} 14), 46.42(\mathrm{CH}, \mathrm{C} 9, \mathrm{C} 13), 40.93\left(\mathrm{CH}_{2}, \mathrm{C} 4\right), 38.45\left(\mathrm{CH}_{2}, \mathrm{C} 1\right)$, 37.36 (C, C10), $33.27\left(\mathrm{CH}_{2}, \mathrm{C} 16\right), 32.11\left(\mathrm{CH}_{2}, \mathrm{C} 2\right), 30.02\left(\mathrm{CH}_{2}, \mathrm{C} 12\right), 22.23\left(\mathrm{CH}_{2}, \mathrm{C} 15\right), 20.65\left(\mathrm{CH}_{2}\right.$, C11), $16.53\left(\mathrm{CH}_{3}, \mathrm{C} 19\right), 14.25\left(\mathrm{CH}_{3}, \mathrm{C} 18\right)$; ESI-MS $(+) \mathrm{m} / z .527(\mathrm{M}+\mathrm{H})^{+}, 590\left(\mathrm{M}^{+} \mathrm{CH}_{3} \mathrm{CN}+\mathrm{Na}\right)^{+}$.

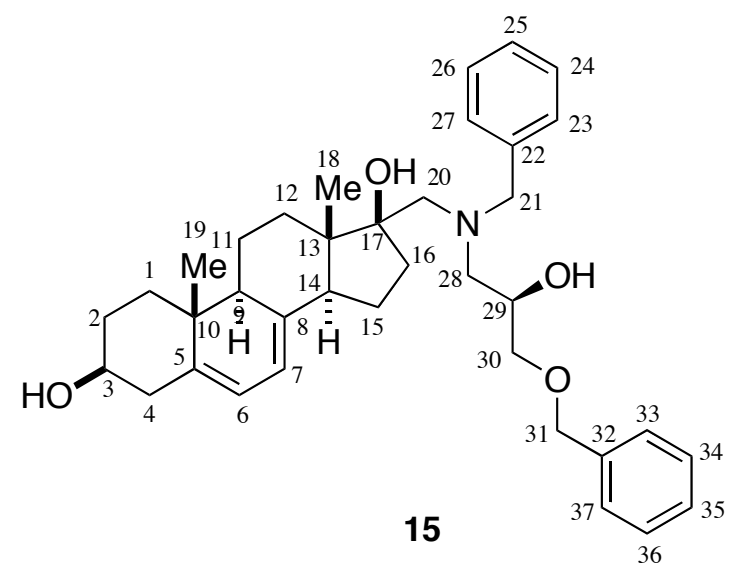

17a-[ $N$-Benzyl- $N$-[3-benzyloxy-(2R)-2-hydroxypropyl]aminomethyl]androsta-5,7-dien-3b,17b-diol (15): mp 99-100 ${ }^{\circ} \mathrm{C}$; IR (thin film) $3363,2933,2862,911,730 \mathrm{~cm}^{-1} ;{ }^{1} \mathrm{H}$ NMR $\left(500 \mathrm{MHz}, \mathrm{CDCl}_{3}\right) \delta$ 7.36-7.27 (m, 10H, C23-27-H, C33-37-H), 5.57 (dd, 1H, $J=5.4,2.4 \mathrm{~Hz}, \mathrm{C} 6-\mathrm{H}), 5.37$ (dt, 1H, $J=5.4$, 
$2.5 \mathrm{~Hz}, \mathrm{C} 7-\mathrm{H}), 4.51$ (s, 2H, C31-H $), 3.95$ (br.s, 1H, C29-H), 3.79 (s, 2H, C21- $\mathrm{H}_{2}$ ), 3.67-3.59 (m, 1H, C3$\mathrm{H}), 3.47-3.41\left(\mathrm{~m}, 2 \mathrm{H}, \mathrm{C} 30-\mathrm{H}_{2}\right), 2.82(\mathrm{~d}, 1 \mathrm{H}, J=13.2 \mathrm{~Hz}, \mathrm{C} 20-\mathrm{H}), 2.72$ (br.s, $\left.2 \mathrm{H}, \mathrm{C} 28-\mathrm{H}_{2}\right), 2.64$ (d, $1 \mathrm{H}, J$ $=13.7 \mathrm{~Hz}, \mathrm{C} 20-\mathrm{H}), 2.47(\mathrm{ddd}, 1 \mathrm{H}, J=14.2,4.4,2.0 \mathrm{~Hz}, \mathrm{C} 4-\mathrm{H}), 2.28(\mathrm{t}, 1 \mathrm{H}, J=13.5 \mathrm{~Hz}, \mathrm{C} 4-\mathrm{H})$, 2.25-2.19 (m, 1H, C16-H), 1.95-1.88 (m, 2H, C9-H, C14-H), 1.89-1.86 (m, 2H, C1-H, C2-H), 1.74-1.66 (m, 1H, C11-H), 1.68-1.58 (m, 4H, C11-H, C12-H, C15-H, C16-H), 1.60-1.46 (m, 2H, C2-H, C15-H), $1.32-1.25(\mathrm{~m}, 2 \mathrm{H}, \mathrm{C} 1-\mathrm{H}, \mathrm{C} 12-\mathrm{H}), 0.95\left(\mathrm{~s}, 3 \mathrm{H}, \mathrm{C} 19-\mathrm{H}_{3}\right), 0.81\left(\mathrm{~s}, 3 \mathrm{H}, \mathrm{C} 18-\mathrm{H}_{3}\right) ;{ }^{13} \mathrm{C} \mathrm{NMR}(125 \mathrm{MHz}$, $\left.\mathrm{CDCl}_{3}\right) \delta 141.21$ (C, C8), 140.32 (C, C5), 139.16 (C, C22), 138.06 (C, C32), 129.21 (CH, C25), 128.62, 128.01, 127.97 (CH x 3, C23, C24, C26, C27, C33, C34, C36, C37), 127.49 (CH, C35), 119.63 (CH, C6), $116.78(\mathrm{CH}, \mathrm{C} 7), 83.33(\mathrm{C}, \mathrm{C} 17), 73.66\left(\mathrm{CH}_{2}, \mathrm{C} 31\right), 72.74\left(\mathrm{CH}_{2}, \mathrm{C} 30\right), 70.55(\mathrm{CH}, \mathrm{C} 3), 68.75(\mathrm{CH}, \mathrm{C} 29)$, $62.87\left(\mathrm{CH}_{2}, \mathrm{C} 21\right), 60.68\left(\mathrm{CH}_{2}, \mathrm{C} 20\right), 59.19\left(\mathrm{CH}_{2}, \mathrm{C} 28\right), 49.06(\mathrm{CH}, \mathrm{C} 14), 46.59(\mathrm{CH}, \mathrm{C} 9), 46.14(\mathrm{C}$, C13), $40.96\left(\mathrm{CH}_{2}, \mathrm{C} 4\right), 38.53\left(\mathrm{CH}_{2}, \mathrm{C} 1\right), 37.34(\mathrm{C}, \mathrm{C} 10), 34.73\left(\mathrm{CH}_{2}, \mathrm{C} 16\right), 32.15\left(\mathrm{CH}_{2}, \mathrm{C} 2\right), 31.07\left(\mathrm{CH}_{2}\right.$, C12), $22.49\left(\mathrm{CH}_{2}, \mathrm{C} 15\right), 20.87\left(\mathrm{CH}_{2}, \mathrm{C} 11\right), 16.50\left(\mathrm{CH}_{3}, \mathrm{C} 19\right), 14.77\left(\mathrm{CH}_{3}, \mathrm{C} 18\right)$; ESI-MS (+) $m / z 572$ $(\mathrm{M}+\mathrm{H})^{+}$.

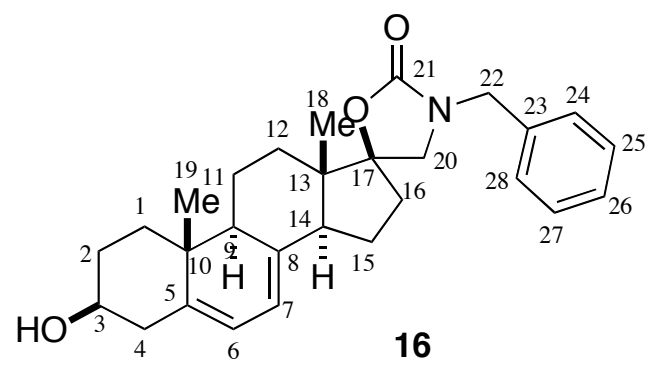

(5S)-3-Benzyloxazolidin-2-one-5-spiro-17'-(3'b-hydroxyandrosta-5',7'-diene) (16): mp $165-166{ }^{\circ} \mathrm{C}$; IR (thin film) 3427, 2930, 2877, 1735, 1070, 1049, 736, $702 \mathrm{~cm}^{-1} ;{ }^{1} \mathrm{H}$ NMR $\left(500 \mathrm{MHz}, \mathrm{CDCl}_{3}\right) \delta 7.36(\mathrm{t}$, $2 \mathrm{H}, J=7.3 \mathrm{~Hz}, \mathrm{C} 25-\mathrm{H}, \mathrm{C} 27-\mathrm{H}), 7.33(\mathrm{t}, 1 \mathrm{H}, J=7.3 \mathrm{~Hz}, \mathrm{C} 26-\mathrm{H}), 7.28$ (d, 2H, $J=7.3 \mathrm{~Hz}, \mathrm{C} 24-\mathrm{H}, \mathrm{C} 28-\mathrm{H})$, $5.55(\mathrm{dd}, 1 \mathrm{H}, J=5.4,2.4 \mathrm{~Hz}, \mathrm{C} 6-\mathrm{H}), 5.41(\mathrm{dt}, 1 \mathrm{H}, J=5.4,2.5 \mathrm{~Hz}, \mathrm{C} 7-\mathrm{H}), 3.62(\mathrm{tt}, 1 \mathrm{H}, J=11.2,3.9 \mathrm{~Hz}$, C3-H), 3.51 (d, 1H, $J=9.3 \mathrm{~Hz}, \mathrm{C} 20-\mathrm{H}), 3.03$ (d, 1H, $J=9.3 \mathrm{~Hz}, \mathrm{C} 20-\mathrm{H}), 2.47$ (ddd, 1H, $J=14.2,4.4,2.0$ $\mathrm{Hz}, \mathrm{C} 4-\mathrm{H}), 2.35$ (ddd, 1H, $J=15.1,12.2,3.9 \mathrm{~Hz}, \mathrm{C} 16-\mathrm{H}), 2.28$ (t, 1H, $J=12.5 \mathrm{~Hz}, \mathrm{C} 4-\mathrm{H}), 1.92-1.85$ (m, 4H, C1-H, C2-H, C9-H, C16-H), 1.83-1.76 (m, 1H, C14-H), 1.75-1.70 (m, 1H, C15-H), 1.70-1.59 (m, 4H, C1-H, C11-H, C12-H, C15-H), 1.54-1.46 (m, 1H, C2-H), 1.30-1.24 (m, 2H, C1-H, C3-OH), $1.21-1.16(\mathrm{~m}, 1 \mathrm{H}, \mathrm{C} 12-\mathrm{H}), 0.94\left(\mathrm{~s}, 3 \mathrm{H}, \mathrm{C} 19-\mathrm{H}_{3}\right), 0.90\left(\mathrm{~s}, 3 \mathrm{H}, \mathrm{C} 18-\mathrm{H}_{3}\right) ;{ }^{13} \mathrm{C} \mathrm{NMR}\left(125 \mathrm{MHz}, \mathrm{CDCl}_{3}\right)$

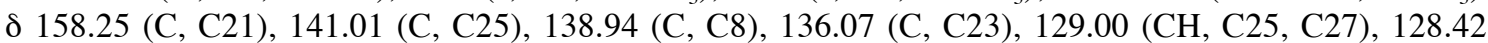
(CH, C24, C28), 128.17 (CH, C26), $119.46(\mathrm{CH}, \mathrm{C} 6), 117.92(\mathrm{CH}, \mathrm{C} 7), 89.60$ (C, C17), 70.47 (CH, C3), $53.00\left(\mathrm{CH}_{2}, \mathrm{C} 20\right), 48.57\left(\mathrm{CH}_{2}, \mathrm{C} 22\right), 47.70(\mathrm{CH}, \mathrm{C} 14), 46.19(\mathrm{CH}, \mathrm{C} 9), 45.33(\mathrm{C}, \mathrm{C} 13), 40.92\left(\mathrm{CH}_{2}, \mathrm{C} 4\right)$, $38.46\left(\mathrm{CH}_{2}, \mathrm{C} 1\right), 37.33(\mathrm{C}, \mathrm{C} 10), 36.11\left(\mathrm{CH}_{2}, \mathrm{C} 16\right), 32.11\left(\mathrm{CH}_{2}, \mathrm{C} 2\right), 30.94\left(\mathrm{CH}_{2}, \mathrm{C} 12\right), 21.87\left(\mathrm{CH}_{2}\right.$, C15), $20.48\left(\mathrm{CH}_{2}, \mathrm{C} 11\right), 16.42\left(\mathrm{CH}_{3}, \mathrm{C} 19\right), 14.71\left(\mathrm{CH}_{3}, \mathrm{C} 18\right)$; ESI-MS (+) $\mathrm{m} / z, 434(\mathrm{M}+\mathrm{H})^{+}, 475$ $\left(\mathrm{M}+\mathrm{CH}_{3} \mathrm{CN}+\mathrm{H}\right)^{+}, 497\left(\mathrm{M}+\mathrm{CH}_{3} \mathrm{CN}+\mathrm{Na}\right)^{+}$.

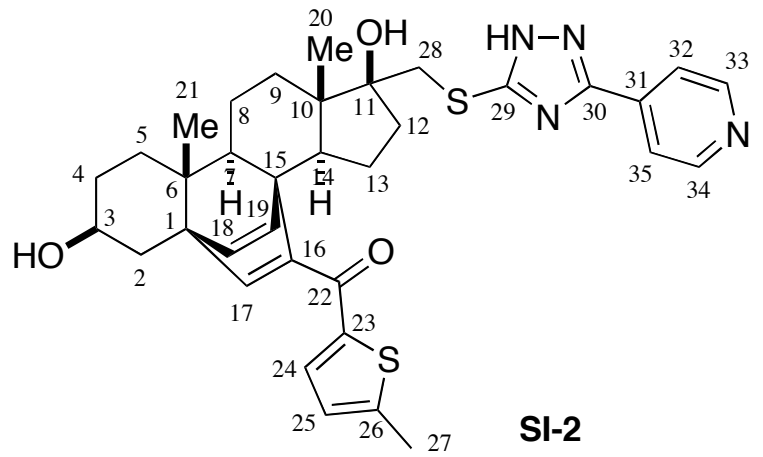

$(1 R, 3 S, 6 R, 7 R, 10 S, 11 S, 14 S, 15 R)-3,11-D i h y d r o x y-6,10-d i m e t h y l-11-[5-(4-p y r i d y l)-1 H-[1,2,4]$ triazol-3ylthiomethyl]pentacyclo[13.2.2.0 $\left.{ }^{1,6} \cdot 0^{7,15} \cdot 0^{10,14}\right]$ nonadeca-16,18-dien-16-yl 5-methylthienyl ketone (SI2): IR (thin film) 3362, 2932, 2868, 1610, 1448, $733 \mathrm{~cm}^{-1} ;{ }^{1} \mathrm{H}$ NMR $\left(500 \mathrm{MHz}, \mathrm{CDCl}_{3}\right) \delta 8.70$ (br.s, $2 \mathrm{H}$, C33-H, C34-H), 7.98 (br.s, 2H, C32-H, C35-H), 7.21 (d, 1H, J = 3.9 Hz, C24-H), 6.77 (d, 1H, J = 3.9 Hz, C25-H), $6.52(\mathrm{~s}, 1 \mathrm{H}, \mathrm{C} 17-\mathrm{H}), 6.38(\mathrm{~d}, 1 \mathrm{H}, J=7.8 \mathrm{~Hz}, \mathrm{C} 19-\mathrm{H}), 6.25(\mathrm{~d}, 1 \mathrm{H}, J=7.8 \mathrm{~Hz}, \mathrm{C} 18-\mathrm{H}), 4.00$ (tt, $1 \mathrm{H}, J=11.2,5.4 \mathrm{~Hz}, \mathrm{C} 3-\mathrm{H}), 3.62(\mathrm{~d}, 1 \mathrm{H}, J=14.2 \mathrm{~Hz}, \mathrm{C} 28-\mathrm{H}), 3.44(\mathrm{~d}, 1 \mathrm{H}, J=14.7 \mathrm{~Hz}, \mathrm{C} 28-\mathrm{H}), 3.04$ 
(dd, $1 \mathrm{H}, J=12.7,7.3 \mathrm{~Hz}, \mathrm{C} 14-\mathrm{H}), 2.53\left(\mathrm{~s}, 3 \mathrm{H}, \mathrm{C} 27-\mathrm{H}_{3}\right), 2.40$ (dd, 1H, J = 13.2, $\left.4.4 \mathrm{~Hz}, \mathrm{C} 2-\mathrm{H}\right), 2.18$ (ddd, $1 \mathrm{H}, J=14.7,9.3,5.9 \mathrm{~Hz}, \mathrm{C} 12-\mathrm{H}), 2.09(\mathrm{t}, 1 \mathrm{H}, J=12.2 \mathrm{~Hz}, \mathrm{C} 2-\mathrm{H}), 1.83(\mathrm{t}, 1 \mathrm{H}, J=12.7 \mathrm{~Hz}, \mathrm{C} 12-\mathrm{H})$, 1.80-1.65 (m, 3H, C4-H, C9-H), 1.66-1.56 (m, 3H, C5-H, C8-H, C13-H), 1.46 (t, 1H, J = 11.5 Hz, C9H), 1.33-1.28 (m, 3H, C7-H, C8-H, C13-H), 1.27 (s, 2H, C3-OH, C11-OH), 1.16 (s, 3H, C20-H $), 1.13$ $(\mathrm{td}, 1 \mathrm{H}, J=13.7,3.9 \mathrm{~Hz}, \mathrm{C} 5-\mathrm{H}), 0.97\left(\mathrm{~s}, 3 \mathrm{H}, \mathrm{C} 21-\mathrm{H}_{3}\right) ;{ }^{13} \mathrm{C} \mathrm{NMR}\left(125 \mathrm{MHz} \mathrm{CDCl}_{3}\right) \delta 189.05(\mathrm{C}, \mathrm{C} 22)$, 159.62 (C, C31), 156.61 (C, C29), 151.38 (C, C26), 150.07 (CH, C16, C33, C34), 145.66 (CH, C17), 142.87 (C, C23), 141.82 (CH, C18), 138.15 (C, C30), 136.08 (CH, C24), 132.22 (CH, C19), 127.10 (CH, C25), 120.87 (CH, C32, C35), 83.70 (C, C11), 68.17 (CH, C3), $54.92(\mathrm{CH}, \mathrm{C} 7), 52.65$ (C, C15), 50.61 $(\mathrm{C}, \mathrm{C} 1), 47.03(\mathrm{C}, \mathrm{C} 10), 43.16\left(\mathrm{CH}_{2}, \mathrm{C} 6, \mathrm{C} 28\right), 42.38(\mathrm{CH}, \mathrm{C} 14), 38.12\left(\mathrm{CH}_{2}, \mathrm{C} 5\right), 36.74\left(\mathrm{CH}_{2}, \mathrm{C} 2\right)$, $35.36\left(\mathrm{CH}_{2}, \mathrm{C} 12\right), 32.28\left(\mathrm{CH}_{2}, \mathrm{C} 9\right), 30.90\left(\mathrm{CH}_{2}, \mathrm{C} 4\right), 24.66\left(\mathrm{CH}_{2}, \mathrm{C} 8\right), 22.56\left(\mathrm{CH}_{2}, \mathrm{C} 13\right), 18.81\left(\mathrm{CH}_{3}\right.$, $\mathrm{C} 21), 16.37\left(\mathrm{CH}_{3}, \mathrm{C} 27\right), 15.29\left(\mathrm{CH}_{3}, \mathrm{C} 20\right)$; ESI-MS $(+) \mathrm{m} / z .629(\mathrm{M}+\mathrm{H})^{+}$.

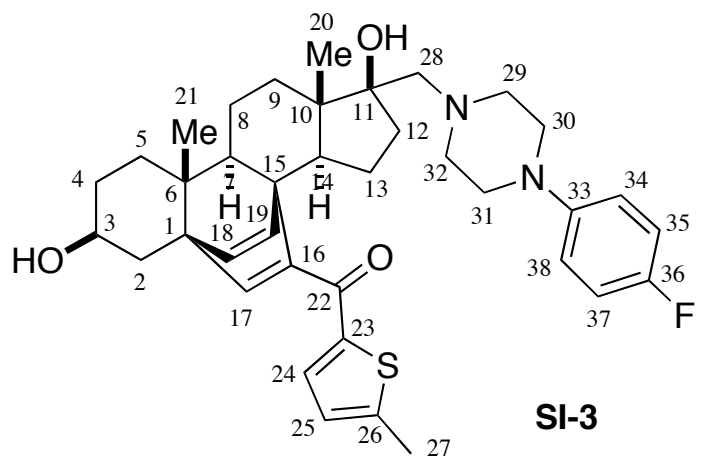

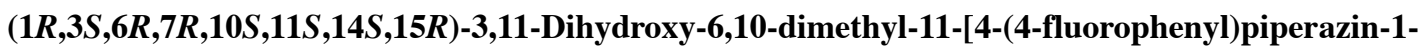
ylmethyl $]$ pentacyclo[13.2.2.1 $\left.{ }^{1,6} \cdot 0^{7,15} \cdot 0^{10,14}\right]$ nonadeca-16,18-dien-16-yl 5-methylthienyl ketone (SI-3): IR (thin film) 3441, 2929, 2873, 1613, 1509, 1451, 1233, $747 \mathrm{~cm}^{-1} ;{ }^{1} \mathrm{H}$ NMR (500 MHz, CDCl $\left.{ }_{3}\right) \delta 7.17$ $(\mathrm{d}, 1 \mathrm{H}, J=3.9 \mathrm{~Hz}, \mathrm{C} 24-\mathrm{H}), 6.96$ (t, 2H, $J=8.8 \mathrm{~Hz}, \mathrm{C} 35-\mathrm{H}, \mathrm{C} 37-\mathrm{H}), 6.87$ (dd, 2H, J = 8.8, 4.9 Hz, C34-H, $\mathrm{C} 38-\mathrm{H}), 6.74(\mathrm{~d}, 1 \mathrm{H}, J=3.7 \mathrm{~Hz}, \mathrm{C} 25-\mathrm{H}), 6.46(\mathrm{~s}, 1 \mathrm{H}, \mathrm{C} 17-\mathrm{H}), 6.38(\mathrm{~d}, 1 \mathrm{H}, J=7.8 \mathrm{~Hz}, \mathrm{C} 19-\mathrm{H}), 6.20(\mathrm{~d}$, $1 \mathrm{H}, J=7.8 \mathrm{~Hz}, \mathrm{C} 18-\mathrm{H}), 3.96(\mathrm{tt}, 1 \mathrm{H}, J=11.2,5.4 \mathrm{~Hz}, \mathrm{C} 3-\mathrm{H}), 3.11\left(\mathrm{~s}, 4 \mathrm{H}, \mathrm{C} 30-\mathrm{H}_{2}, \mathrm{C} 31-\mathrm{H}_{2}\right), 2.93-2.87$ (m, 2H, C29-H, C32-H), 2.83 (d, 1H, J = 13.2 Hz, C28-H), 2.82-2.78 (m, 3H, C14-H, C29-H, C32-H), $2.59(\mathrm{~d}, 1 \mathrm{H}, J=13.2 \mathrm{~Hz}, \mathrm{C} 28-\mathrm{H}), 2.52\left(\mathrm{~s}, 3 \mathrm{H}, \mathrm{C} 27-\mathrm{H}_{3}\right), 2.36(\mathrm{dd}, 1 \mathrm{H}, J=12.7,3.9 \mathrm{~Hz}, \mathrm{C} 2-\mathrm{H}), 2.05$ (t, $1 \mathrm{H}, J=12.2 \mathrm{~Hz}, \mathrm{C} 2-\mathrm{H}), 1.91-1.82(\mathrm{~m}, 1 \mathrm{H}, \mathrm{C} 12-\mathrm{H}), 1.85-1.79(\mathrm{~m}, 1 \mathrm{H}, \mathrm{C} 12-\mathrm{H}), 1.76-1.72(\mathrm{~m}, 1 \mathrm{H}, \mathrm{C} 4-$ H), 1.65 (qd, $J=12.5,4.2 \mathrm{~Hz}, \mathrm{C} 4-\mathrm{H}), 1.57-1.49\left(\mathrm{~m}, 5 \mathrm{H}, \mathrm{C} 5-\mathrm{H}, \mathrm{C} 8-\mathrm{H}_{2}, \mathrm{C} 9-\mathrm{H}, \mathrm{C} 13-\mathrm{H}\right), 1.45-1.39$ (m, $1 \mathrm{H}$, C9-H), 1.29-1.22 (m, 3H, C7-H, C13-H, C3-OH), 1.11 (td, 1H, J = 13.7, $3.9 \mathrm{~Hz}, \mathrm{C} 5-\mathrm{H}), 1.07$ (s, 3H, $\left.\mathrm{C} 20-\mathrm{H}_{3}\right), 0.95\left(\mathrm{~s}, 3 \mathrm{H}, \mathrm{C} 21-\mathrm{H}_{3}\right) ;{ }^{13} \mathrm{C} \mathrm{NMR}\left(125 \mathrm{MHz}, \mathrm{CDCl}_{3}\right) \delta 188.58(\mathrm{C}, \mathrm{C} 22), 157.37$ (d, J = 238.0 Hz, C, C36), 150.58 (C, C26), 150.26 (C, C16), 148.04 (C, C33), 145.01 (CH, C17), 143.22 (C, C23), 141.38 $(\mathrm{CH}, \mathrm{C} 18), 135.52(\mathrm{CH}, \mathrm{C} 24), 132.61(\mathrm{CH}, \mathrm{C} 19), 126.85(\mathrm{CH}, \mathrm{C} 25), 117.95(\mathrm{~d}, J=6.9 \mathrm{~Hz}, \mathrm{CH}, \mathrm{C} 34$, C38), 115.69 (d, J = 22.1 Hz, CH, C35, C37), 80.52 (C, C11), $68.18(\mathrm{CH}, \mathrm{C} 3), 63.19\left(\mathrm{CH}_{2}, \mathrm{C} 28\right), 55.33$ $\left(\mathrm{CH}_{2}, \mathrm{C} 29, \mathrm{C} 32\right), 54.93(\mathrm{CH}, \mathrm{C} 7), 52.75(\mathrm{C}, \mathrm{C} 15), 50.62\left(\mathrm{CH}_{2}, \mathrm{C} 30, \mathrm{C} 31\right), 50.43(\mathrm{C}, \mathrm{C} 1), 45.60(\mathrm{C}, \mathrm{C} 10)$, 43.19 (C, C6), $41.86(\mathrm{CH}, \mathrm{C} 14), 38.07\left(\mathrm{CH}_{2}, \mathrm{C} 5\right), 36.82\left(\mathrm{CH}_{2}, \mathrm{C} 2\right), 36.61\left(\mathrm{CH}_{2}, \mathrm{C} 12\right), 32.18\left(\mathrm{CH}_{2}, \mathrm{C} 9\right)$, $30.92\left(\mathrm{CH}_{2}, \mathrm{C} 4\right), 24.83\left(\mathrm{CH}_{2}, \mathrm{C} 8\right), 22.62\left(\mathrm{CH}_{2}, \mathrm{C} 13\right), 18.79\left(\mathrm{CH}_{3}, \mathrm{C} 21\right), 16.31\left(\mathrm{CH}_{3}, \mathrm{C} 27\right), 15.23\left(\mathrm{CH}_{3}\right.$, C20); ESI-MS (+) $m / z, 631(\mathrm{M}+\mathrm{H})^{+}$.

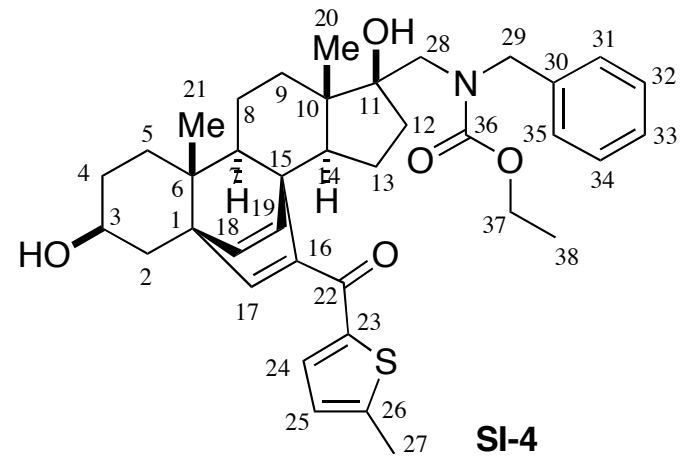

$N$-Benzyl- $N$-[(1R,3S,6R,7R,10S,11S,14S,15R)-3,11-dihydroxy-6,10-dimethyl-16-(5-methylthiophene- 
2-carbonyl)pentacyclo[13.2.2. $0^{1,6} \cdot 0^{7,15} \cdot 0^{10,14}$ nonadeca-16,18-dien-11-ylmethyl]carbamic acid ethyl ester (SI-4): IR (thin film) 3398, 2982, 2923, 2870, 1670, 1617, 1449, 1265, $735 \mathrm{~cm}^{-1}$; ${ }^{1} \mathrm{H}$ NMR (500 $\left.\mathrm{MHz}, \mathrm{CDCl}_{3}\right) \delta 7.50(\mathrm{~d}, 2 \mathrm{H}, J=6.8 \mathrm{~Hz}, \mathrm{C} 31-\mathrm{H}, \mathrm{C} 35-\mathrm{H}), 7.40(\mathrm{t}, 2 \mathrm{H}, J=6.8 \mathrm{~Hz}, \mathrm{C} 32-\mathrm{H}, \mathrm{C} 34-\mathrm{H}), 7.32(\mathrm{t}$, $1 \mathrm{H}, J=6.8 \mathrm{~Hz}, \mathrm{C} 33-\mathrm{H}), 7.18$ (br.s, $1 \mathrm{H}, \mathrm{C} 24-\mathrm{H}), 6.75(\mathrm{~d}, 1 \mathrm{H}, J=3.4 \mathrm{~Hz}, \mathrm{C} 25-\mathrm{H}), 6.44(\mathrm{~s}, 1 \mathrm{H}, \mathrm{C} 17-\mathrm{H})$, $6.38(\mathrm{~d}, 1 \mathrm{H}, J=7.3 \mathrm{~Hz}, \mathrm{C} 19-\mathrm{H}), 6.20(\mathrm{~d}, 1 \mathrm{H}, J=7.8 \mathrm{~Hz}, \mathrm{C} 18-\mathrm{H}), 4.97(\mathrm{~d}, 1 \mathrm{H}, J=14.7 \mathrm{~Hz}, \mathrm{C} 29-\mathrm{H}), 4.73$ (br.s, 1H, C11-OH), 4.32 (br.s, 1H, C3-OH), 4.24-4.23 (m, 2H, C37- $\mathrm{H}_{2}$ ), 4.03 (d, 1H, J = 15.6 Hz, C29H), 3.95 (tt, $1 \mathrm{H}, J=11.2,4.9 \mathrm{~Hz}, \mathrm{C} 3-\mathrm{H}), 3.73(\mathrm{~d}, 1 \mathrm{H}, J=15.1 \mathrm{~Hz}, \mathrm{C} 28-\mathrm{H}), 3.22(\mathrm{~d}, 1 \mathrm{H}, J=15.1 \mathrm{~Hz}$, C28-H), $2.95(\mathrm{t}, 1 \mathrm{H}, J=6.8 \mathrm{~Hz}, \mathrm{C} 14-\mathrm{H}), 2.52\left(\mathrm{~s}, 3 \mathrm{H}, \mathrm{C} 27-\mathrm{H}_{3}\right), 2.36(\mathrm{dd}, 1 \mathrm{H}, J=12.7,4.9 \mathrm{~Hz}, \mathrm{C} 2-\mathrm{H})$, $2.04(\mathrm{t}, 1 \mathrm{H}, J=12.7 \mathrm{~Hz}, \mathrm{C} 2-\mathrm{H}), 1.91-1.85(\mathrm{~m}, 1 \mathrm{H}, \mathrm{C} 12-\mathrm{H}), 1.74-1.61(\mathrm{~m}, 2 \mathrm{H}, \mathrm{C} 4-\mathrm{H}, \mathrm{C} 12-\mathrm{H}), 1.60-1.46$ (m, 5H, C4-H, C5-H, C8-H, C9-H, C13-H), $1.30\left(\mathrm{t}, 3 \mathrm{H}, J=7.3 \mathrm{~Hz}, \mathrm{C} 38-\mathrm{H}_{3}\right), 1.27-1.22(\mathrm{~m}, 3 \mathrm{H}, \mathrm{C} 8-\mathrm{H}$, $\mathrm{C} 9-\mathrm{H}, \mathrm{C} 13-\mathrm{H}), 1.10(\mathrm{td}, 1 \mathrm{H}, J=13.7,2.9 \mathrm{~Hz}, \mathrm{C} 5-\mathrm{H}), 1.06\left(\mathrm{~s}, 3 \mathrm{H}, \mathrm{C} 20-\mathrm{H}_{3}\right), 0.92\left(\mathrm{~s}, 3 \mathrm{H}, \mathrm{C} 21-\mathrm{H}_{3}\right) ;{ }^{13} \mathrm{C}$ NMR (125 MHz, CDCl $)_{3} \delta 188.36$ (C, C22), 159.83 (C, C36), 150.52 (C, C26), 150.25 (C, C16), 144.04 (CH, C17), 143.41 (C, C23), 141.58 (CH, C18), 137.70 (C, C30), 135.31 (CH, C24), 132.22 (CH, C19), $128.80(\mathrm{CH}, \mathrm{C} 32, \mathrm{C} 34), 128.44(\mathrm{CH}, \mathrm{C} 31, \mathrm{C} 35), 127.68$ (CH, C33), $126.84(\mathrm{CH}, \mathrm{C} 25), 84.24$ (C, C11), $68.11(\mathrm{CH}, \mathrm{C} 3), 62.48\left(\mathrm{CH}_{2}, \mathrm{C} 37\right), 55.80\left(\mathrm{CH}_{2}, \mathrm{C} 28\right), 54.94(\mathrm{CH}, \mathrm{C} 7), 53.96\left(\mathrm{CH}_{2}, \mathrm{C} 29\right), 52.46(\mathrm{C}, \mathrm{C} 15)$, 50.43 (C, C1), 45.72 (C, C10), 43.11 (C, C6), $41.38(\mathrm{CH}, \mathrm{C} 14), 38.02\left(\mathrm{CH}_{2}, \mathrm{C} 5\right), 36.82\left(\mathrm{CH}_{2}, \mathrm{C} 2\right), 31.75$ $\left(\mathrm{CH}_{2}, \mathrm{C} 12\right), 30.86\left(\mathrm{CH}_{2}, \mathrm{C} 4, \mathrm{C} 9\right), 24.39\left(\mathrm{CH}_{2}, \mathrm{C} 8\right), 22.23\left(\mathrm{CH}_{2}, \mathrm{C} 13\right), 18.72\left(\mathrm{CH}_{3}, \mathrm{C} 21\right), 16.30\left(\mathrm{CH}_{3}\right.$, C27), $14.80\left(\mathrm{CH}_{3}, \mathrm{C} 38\right), 14.36\left(\mathrm{CH}_{3}, \mathrm{C} 20\right)$; ESI-MS $(+) \mathrm{m} / z 630(\mathrm{M}+\mathrm{H})^{+}, 652(\mathrm{M}+\mathrm{Na})^{+}$.

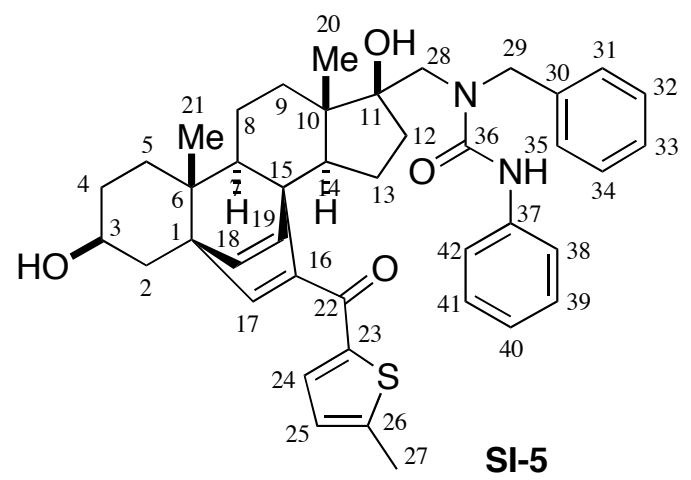

1-Benzyl-1-[(1R,3S,6R,7R,10S,11S,14S,15R)-3,11-dihydroxy-6,10-dimethyl-16-(5-methylthiophene2-carbonyl)pentacyclo[13.2.2.0 $\left.0^{1,6} \cdot 0^{7,15} \cdot 0^{10,14}\right]$ nonadeca-16,18-dien-11-ylmethyl]-3-phenylurea (SI-5): IR (thin film) 3303, 3048, 2987, 1654, 1613, 1445, 1266, $737 \mathrm{~cm}^{-1} ;{ }^{1} \mathrm{H}$ NMR (500 MHz, $\mathrm{CDCl}_{3}$ ) $\delta 7.63$ (d, 2H, $J=7.3 \mathrm{~Hz}, \mathrm{C} 31-\mathrm{H}, \mathrm{C} 35-\mathrm{H}), 7.40$ (t, $2 \mathrm{H}, J=7.8 \mathrm{~Hz}, \mathrm{C} 32-\mathrm{H}, \mathrm{C} 34-\mathrm{H}), 7.37$ (d, 2H, $J=8.8 \mathrm{~Hz}, \mathrm{C} 38-$ H, C42-H), 7.31 (t, 1H, $J=7.3 \mathrm{~Hz}, \mathrm{C} 33-\mathrm{H}), 7.24$ (t, 2H, $J=7.8 \mathrm{~Hz}, \mathrm{C} 39-\mathrm{H}, \mathrm{C} 41-\mathrm{H}), 7.19$ (d, 1H, $J=3.9$ $\mathrm{Hz}, \mathrm{C} 24-\mathrm{H}), 6.98(\mathrm{t}, 1 \mathrm{H}, J=7.3 \mathrm{~Hz}, \mathrm{C} 40-\mathrm{H}), 6.76(\mathrm{dd}, 1 \mathrm{H}, J=3.9,1.0 \mathrm{~Hz}, \mathrm{C} 25-\mathrm{H}), 6.47(\mathrm{~s}, 1 \mathrm{H}, \mathrm{C} 17-\mathrm{H})$, $6.35(\mathrm{~d}, 1 \mathrm{H}, J=7.8 \mathrm{~Hz}, \mathrm{C} 19-\mathrm{H}), 6.22(\mathrm{~d}, 1 \mathrm{H}, J=7.8 \mathrm{~Hz}, \mathrm{C} 18-\mathrm{H}), 5.09$ (d, $1 \mathrm{H}, J=13.2 \mathrm{~Hz}, \mathrm{C} 29-\mathrm{H}), 4.15$ (d, $1 \mathrm{H}, J=13.2 \mathrm{~Hz}, \mathrm{C} 29-\mathrm{H}), 3.95(\mathrm{tt}, 1 \mathrm{H}, J=11.2,5.4 \mathrm{~Hz}, \mathrm{C} 3-\mathrm{H}), 3.78$ (d, $1 \mathrm{H}, J=16.1 \mathrm{~Hz}, \mathrm{C} 28-\mathrm{H}), 3.37$ $(\mathrm{d}, 1 \mathrm{H}, J=16.1 \mathrm{~Hz}, \mathrm{C} 28-\mathrm{H}), 3.04(\mathrm{dd}, 1 \mathrm{H}, J=11.2,7.8 \mathrm{~Hz}, \mathrm{C} 14-\mathrm{H}), 2.54\left(\mathrm{~s}, 3 \mathrm{H}, \mathrm{C} 27-\mathrm{H}_{3}\right), 2.37$ (dd, $1 \mathrm{H}$, $J=12.7,3.9 \mathrm{~Hz}, \mathrm{C} 2-\mathrm{H}), 2.18-2.14(\mathrm{~m}, 1 \mathrm{H}, \mathrm{C} 12-\mathrm{H}), 2.05(\mathrm{t}, 1 \mathrm{H}, J=12.2 \mathrm{~Hz}, \mathrm{C} 2-\mathrm{H}), 1.75-1.72(\mathrm{~m}, 1 \mathrm{H}$, C4-H), 1.64 (qd, $1 \mathrm{H}, J=12.5,3.9 \mathrm{~Hz}, \mathrm{C} 4-\mathrm{H}), 1.59-1.54$ (m, 3H, C9-H, C12-H, C13-H), 1.53-1.50 (m, $2 \mathrm{H}, \mathrm{C} 5-\mathrm{H}, \mathrm{C} 8-\mathrm{H}), 1.36-1.30$ (m, 2H, C9-H, C13-H), 1.25-1.20 (m, 2H, C7-H, C8-H), 1.09 (td, 1H, J = 13.2, $3.9 \mathrm{~Hz}, \mathrm{C} 5-\mathrm{H}), 1.08\left(\mathrm{~s}, 3 \mathrm{H}, \mathrm{C} 20-\mathrm{H}_{3}\right), 0.93\left(\mathrm{~s}, 3 \mathrm{H}, \mathrm{C} 21-\mathrm{H}_{3}\right) ;{ }^{13} \mathrm{C}$ NMR $\left(125 \mathrm{MHz}, \mathrm{CDCl}_{3}\right) \delta 188.21$ (C, C22), 158.19 (C, C36), 150.82 (C, C26), 149.99 (C, C16), 144.36 (CH, C17), 143.25 (C, C23), 141.97 (CH, C18), 140.03 (C, C37), 138.21 (C, C30), 135.37 (CH, C24), 131.82 (CH, C19), 128.89 (CH, C32, C34, C39, C41), 128.47 ( $\mathrm{CH}, \mathrm{C} 31, \mathrm{C} 35), 127.56(\mathrm{CH}, \mathrm{C} 33), 126.91(\mathrm{CH}, \mathrm{C} 25), 122.53$ (CH, C40), $119.64(\mathrm{CH}, \mathrm{C} 38, \mathrm{C} 42), 85.68$ (C, C11), $68.11(\mathrm{CH}, \mathrm{C} 3), 55.23\left(\mathrm{CH}_{2}, \mathrm{C} 28\right), 54.77(\mathrm{CH}, \mathrm{C} 7), 53.57\left(\mathrm{CH}_{2}\right.$, C29), 52.29 (C, C15), 50.50 (C, C1), 45.80 (C, C10), 43.13 (C, C6), 41.39 (CH, C14), $38.00\left(\mathrm{CH}_{2}, \mathrm{C} 5\right)$, $36.76\left(\mathrm{CH}_{2}, \mathrm{C} 2\right), 32.31\left(\mathrm{CH}_{2}, \mathrm{C} 12\right), 30.85\left(\mathrm{CH}_{2}, \mathrm{C} 4\right), 30.55\left(\mathrm{CH}_{2}, \mathrm{C} 9\right), 24.35\left(\mathrm{CH}_{2}, \mathrm{C} 8\right), 22.23\left(\mathrm{CH}_{2}\right.$, C13), $18.76\left(\mathrm{CH}_{3}, \mathrm{C} 21\right), 16.33\left(\mathrm{CH}_{3}, \mathrm{C} 27\right), 14.44\left(\mathrm{CH}_{3}, \mathrm{C} 20\right)$; ESI-MS (+) $\mathrm{m} / z 677(\mathrm{M}+\mathrm{H})^{+}$. 


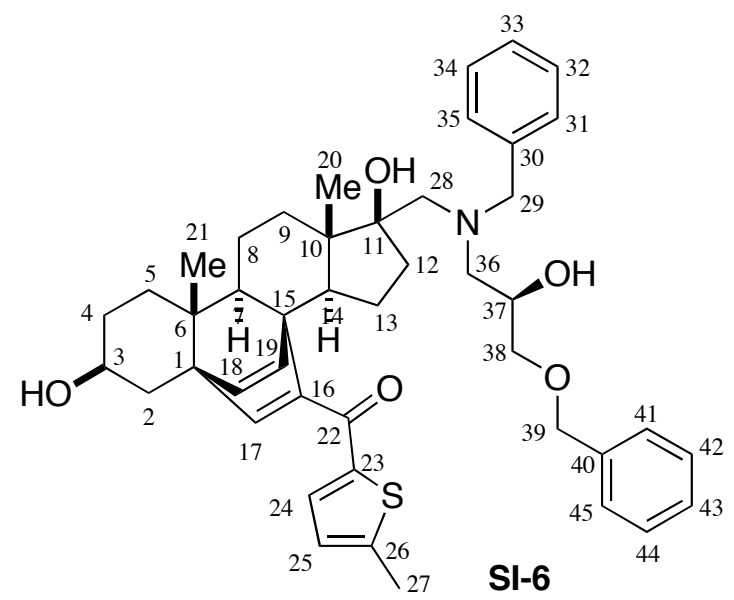

$(1 R, 3 S, 6 R, 7 R, 10 S, 11 S, 14 S, 15 R)-11-[N$-Benzyl- $N$-[3-benzyloxy-(2R)-2-hydroxypropyl]aminomethyl]3,11-dihydroxy-6,10-dimethylpentacyclo $\left[13.2 .2 .0^{1,6} \cdot 0^{7,15} \cdot 0^{10,14}\right]$ n on ad e ca - 16,18 - d i en - 16 - yl 5 methylthienyl ketone (SI-6): IR (thin film) 3398, 2927, 2865, 1610, 1451, 909, $733 \mathrm{~cm}^{-1} ;{ }^{1} \mathrm{H}$ NMR (500 $\mathrm{MHz}_{2} \mathrm{CDCl}_{3}$ ) $\delta 7.39$ (d 2H, J = 7.3 Hz, C31-H, C35-H), 7.35-7.31 (m, 4H, C32-H, C34-H, C42-H, C44H), 7.29-7.27 (m, 3H, C41-H, C43-H, C45-H), $7.25(\mathrm{t}, 1 \mathrm{H}, J=7.3 \mathrm{~Hz}, \mathrm{C} 33-\mathrm{H}), 7.16(\mathrm{~d}, 1 \mathrm{H}, J=3.9 \mathrm{~Hz}$, C24-H), $6.73(\mathrm{~d}, 1 \mathrm{H}, J=3.9 \mathrm{~Hz}, \mathrm{C} 25-\mathrm{H}), 6.44(\mathrm{~s}, 1 \mathrm{H}, \mathrm{C} 17-\mathrm{H}), 6.37$ (d, 1H, J = 7.8 Hz, C19-H), 6.19 (d, $1 \mathrm{H}, J=7.8 \mathrm{~Hz}, \mathrm{C} 18-\mathrm{H}), 4.50\left(\mathrm{~s}, 2 \mathrm{H}, \mathrm{C} 39-\mathrm{H}_{2}\right), 3.98-3.93(\mathrm{~m}, 1 \mathrm{H}, \mathrm{C} 37-\mathrm{H}), 3.94(\mathrm{tt}, 1 \mathrm{H}, J=11.2,5.2 \mathrm{~Hz}$, C3-H), 3.86 (d, 1H, J = 14.2 Hz, C29-H), 3.83 (d, 1H, J = 14.2 Hz, C29-H), 3.43 (dd, 1H, J = 9.8, 3.9 Hz, C38-H), 3.39 (dd, $1 \mathrm{H}, J=9.3,6.4 \mathrm{~Hz}, \mathrm{C} 38-\mathrm{H}), 2.92$ (br.s, 2H, C28- $\mathrm{H}_{2}$ ), 2.88 (dd, $1 \mathrm{H}, J=12.7,7.8 \mathrm{~Hz}$, C14-H), $2.71(\mathrm{dd}, 1 \mathrm{H}, J=13.2,8.3 \mathrm{~Hz}, \mathrm{C} 36-\mathrm{H}), 2.66(\mathrm{t}, 1 \mathrm{H}, J=8.2 \mathrm{~Hz}, \mathrm{C} 36-\mathrm{H}), 2.51\left(\mathrm{~s}, 3 \mathrm{H}, \mathrm{C} 27-\mathrm{H}_{3}\right)$, $2.35(\mathrm{dd}, 1 \mathrm{H}, J=12.2,4.9 \mathrm{~Hz}, \mathrm{C} 2-\mathrm{H}), 2.11-2.06(\mathrm{~m}, 1 \mathrm{H}, \mathrm{C} 12-\mathrm{H}), 2.05(\mathrm{t}, 1 \mathrm{H}, J=12.2 \mathrm{~Hz}, \mathrm{C} 2-\mathrm{H})$, 1.76-1.70 (m, 1H, C4-H), 1.70-1.65 (m, 1H, C12-H), 1.65 (qd, 1H, J = 13.2, 3.9 Hz, C4-H), 1.53 (td, $1 \mathrm{H}$, $J=13.7,3.4 \mathrm{~Hz}, \mathrm{C} 5-\mathrm{H}), 1.52-1.45(\mathrm{~m}, 3 \mathrm{H}, \mathrm{C} 8-\mathrm{H}, \mathrm{C} 9-\mathrm{H}, \mathrm{C} 13-\mathrm{H}), 1.38(\mathrm{t}, 1 \mathrm{H}, J=11.0 \mathrm{~Hz}, \mathrm{C} 9-\mathrm{H})$, 1.26-1.18 (m, 3H, C7-H, C8-H, C13-H), 1.10 (td, 1H, J = 13.7, 3.9 Hz, C5-H), 1.05 (s, 3H, C20-H $)_{3}, 0.94$ (s, 3H, C21-H $\left.{ }_{3}\right) ;{ }^{13} \mathrm{C}$ NMR (125 MHz, $\left.\mathrm{CDCl}_{3}\right) \delta 188.52$ (C, C22), 150.56 (C, C26), 150.32 (C, C16), 144.44 (CH, C17), 143.19 (C, C23), 141.41 (CH, C18), 139.23 (C, C30), 138.17 (C, C40), 135.46 (CH, C24), 132.48 (CH, C19), 129.19 (CH, C31, C35), 128.58, 128.51 (CH x 2, C32, C34, C42, C44), 127.88 $(\mathrm{CH}, \mathrm{C} 41, \mathrm{C} 45), 127.79(\mathrm{CH}, \mathrm{C} 43), 127.28(\mathrm{CH}, \mathrm{C} 33), 126.79(\mathrm{CH}, \mathrm{C} 25), 82.14(\mathrm{C}, \mathrm{C} 11), 73.51\left(\mathrm{CH}_{2}\right.$, C39), $72.90\left(\mathrm{CH}_{2}, \mathrm{C} 38\right), 68.66(\mathrm{CH}, \mathrm{C} 37), 68.06(\mathrm{CH}, \mathrm{C} 3), 62.68\left(\mathrm{CH}_{2}, \mathrm{C} 29\right), 60.67\left(\mathrm{CH}_{2}, \mathrm{C} 28\right), 59.09$ $\left(\mathrm{CH}_{2}, \mathrm{C} 36\right), 54.90(\mathrm{CH}, \mathrm{C} 7), 52.68(\mathrm{C}, \mathrm{C} 15), 50.38(\mathrm{C}, \mathrm{C} 1), 45.60(\mathrm{C}, \mathrm{C} 10), 43.10(\mathrm{C}, \mathrm{C} 6), 41.80(\mathrm{CH}$, $\mathrm{C} 14), 38.02\left(\mathrm{CH}_{2}, \mathrm{C} 5\right), 36.76\left(\mathrm{CH}_{2}, \mathrm{C} 2\right), 34.49\left(\mathrm{CH}_{2}, \mathrm{C} 12\right), 31.55\left(\mathrm{CH}_{2}, \mathrm{C} 9\right), 30.84\left(\mathrm{CH}_{2}, \mathrm{C} 4\right), 24.64$ $\left(\mathrm{CH}_{2}, \mathrm{C} 8\right), 22.43\left(\mathrm{CH}_{2}, \mathrm{C} 13\right), 18.73\left(\mathrm{CH}_{3}, \mathrm{C} 21\right), 16.26\left(\mathrm{CH}_{3}, \mathrm{C} 27\right), 14.94\left(\mathrm{CH}_{3}, \mathrm{C} 20\right)$; ESI-MS $(+) \mathrm{m} / \mathrm{z}$ $722(\mathrm{M}+\mathrm{H})^{+}$.

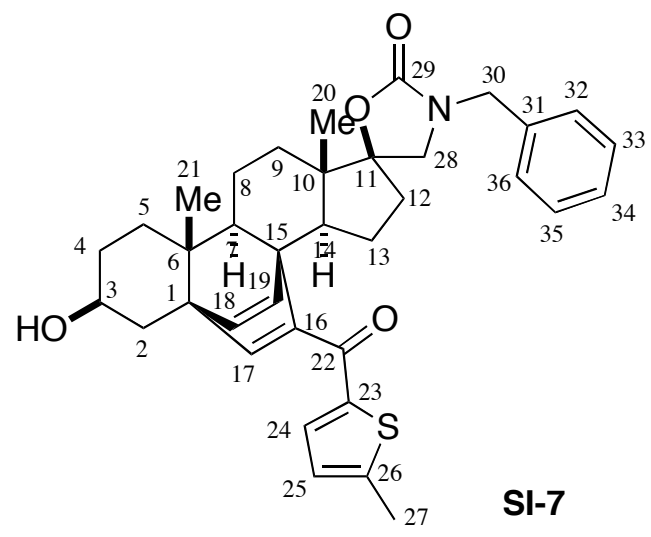

(5S)-3-Benzyloxazolidin-2-one-5spiro-11'-[(1'R,3' $S, 6$ ' $R, 7^{\prime} R, 10$ ' $S, 14 ' S, 15$ ' $\left.R\right)$-6',10'-dimethyl-3'hydroxy-16'-(5-methylthiophene-2-carbonyl)pentacyclo[13.2.2.0 $\left.{ }^{1,6^{\prime}}, 0^{7^{\prime}, 15^{\prime}}, 0^{10^{\prime}, 1^{\prime}}\right]$ nonadeca-16',18'diene] (SI-7): $\mathrm{mp} 157^{\circ} \mathrm{C}$; IR (thin film) 3367, 2991, 2928, 2875, 1751, 1613, 1453, 1273, 1062, 737, 701 $\mathrm{cm}^{-1} ;{ }^{1} \mathrm{H}$ NMR $\left(500 \mathrm{MHz}, \mathrm{CDCl}_{3}\right) \delta 7.36(\mathrm{t}, 2 \mathrm{H}, J=7.3 \mathrm{~Hz}, \mathrm{C} 33-\mathrm{H}, \mathrm{C} 35-\mathrm{H}), 7.32$ (t, 1H, J = 6.8 Hz, C34- 
H), $7.28(\mathrm{~d}, 2 \mathrm{H}, J=7.3 \mathrm{~Hz}, \mathrm{C} 32-\mathrm{H}, \mathrm{C} 36-\mathrm{H}), 7.14(\mathrm{~d}, 1 \mathrm{H}, J=3.9 \mathrm{~Hz}, \mathrm{C} 24-\mathrm{H}), 6.73(\mathrm{~d}, 1 \mathrm{H}, J=3.4 \mathrm{~Hz}$, C25-H), 6.48 (s, 1H, C17-H), 6.31 (d, 1H, J = 7.3 Hz, C19-H), 6.21 (d, 1H, J = 7.8 Hz, C18-H), 4.41 (s, $\left.2 \mathrm{H}, \mathrm{C} 30-\mathrm{H}_{2}\right), 3.95(\mathrm{tt}, 1 \mathrm{H}, J=11.2,5.4 \mathrm{~Hz}, \mathrm{C} 3-\mathrm{H}), 3.65$ (d, $\left.1 \mathrm{H}, J=9.3 \mathrm{~Hz}, \mathrm{C} 28-\mathrm{H}\right), 3.16(\mathrm{~d}, 1 \mathrm{H}, J=9.3$ $\mathrm{Hz}, \mathrm{C} 28-\mathrm{H}), 2.67$ (dd, 1H, J = 12.7, 7.3 Hz, C14-H), 2.51 (s, 3H, C27-H $), 2.35$ (dd, 1H, J = 13.2, 4.4 Hz, C2-H), 2.26 (ddd, 1H, $J=14.2,12.7,3.9 \mathrm{~Hz}, \mathrm{C} 12-\mathrm{H}), 2.04$ (t, 1H, $J=12.2 \mathrm{~Hz}, \mathrm{C} 2-\mathrm{H}), 1.77$ (ddd, 1H, $J=$ 14.2, 9.3, 5.1 Hz, C4-H), 1.75-1.71 (m, 1H, C4-H), 1.65 (td, 1H, J = 13.7, 3.9 Hz, C9-H), 1.59-1.50 (m, 3H, C5-H, C8-H, C13-H), 1.29-1.17 (m, 4H), C7-H, C8-H, C9-H, C13-H), 1.10 (s, 3H, C20-H ), 1.04 (td, $1 \mathrm{H}, J=13.2,4.4 \mathrm{~Hz}, \mathrm{C} 5-\mathrm{H}), 0.93\left(\mathrm{~s}, 3 \mathrm{H}, \mathrm{C} 21-\mathrm{H}_{3}\right) ;{ }^{13} \mathrm{C} \mathrm{NMR}\left(125 \mathrm{MHz}, \mathrm{CDCl}_{3}\right) \delta 188.37(\mathrm{C}, \mathrm{C} 22)$, 158.26 (C, C29), 151.01 (C, C26), 149.31 (C, C16), 145.64 (CH, C17), 142.87 (C, C23), 141.88 (CH, C18), 136.09 (C, C31), 135.68 (CH, C24), 131.77 (CH, C19), 129.00 (CH, C33, C35), 128.32 (CH, C32, C36), 128.09 (CH, C34), 126.96 (CH, C25), 89.11 (C, C11), 68.09 (CH, C3), $54.53(\mathrm{CH}, \mathrm{C} 7), 53.33$ $\left(\mathrm{CH}_{2}, \mathrm{C} 28\right), 52.06(\mathrm{C}, \mathrm{C} 15), 50.50(\mathrm{C}, \mathrm{C} 1), 48.44\left(\mathrm{CH}_{2}, \mathrm{C} 30\right), 45.14(\mathrm{C}, \mathrm{C} 10), 43.30(\mathrm{C}, \mathrm{C} 6), 41.07(\mathrm{CH}$, $\mathrm{C} 14), 37.97\left(\mathrm{CH}_{2}, \mathrm{C} 5\right), 36.68\left(\mathrm{CH}_{2}, \mathrm{C} 2\right), 32.23\left(\mathrm{CH}_{2}, \mathrm{C} 12\right), 31.32\left(\mathrm{CH}_{2}, \mathrm{C} 9\right), 30.84\left(\mathrm{CH}_{2}, \mathrm{C} 4\right), 24.47$ $\left(\mathrm{CH}_{2}, \mathrm{C} 8\right), 21.50\left(\mathrm{CH}_{2}, \mathrm{C} 13\right), 18.75\left(\mathrm{CH}_{3}, \mathrm{C} 21\right), 16.33\left(\mathrm{CH}_{3}, \mathrm{C} 27\right), 14.86\left(\mathrm{CH}_{3}, \mathrm{C} 20\right)$; ESI-MS $(+) \mathrm{m} / z$ $584(\mathrm{M}+\mathrm{H})^{+}$.

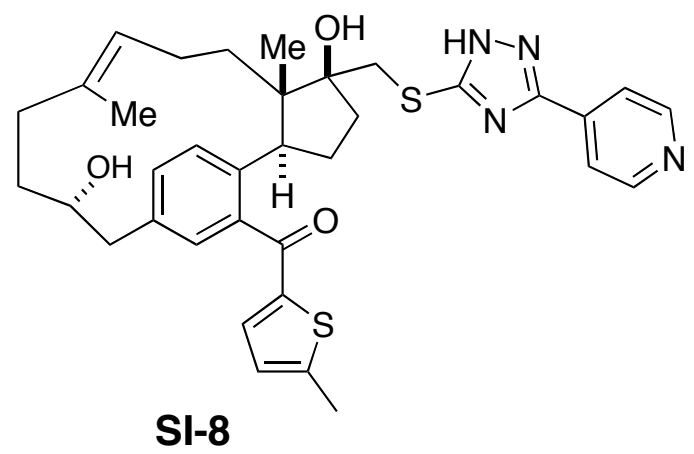

$(2 S, 5 S, 6 S, 13 S, 9 E)-5,13-D i h y d r o x y-6,10-d i m e t h y l-5-[5-(4-p y r i d y l)-1 H-[1,2,4]$ triazol-3-

ylthiomethyl]tricyclo[13.2.2.0 $\left.{ }^{2,6}\right]$ nonadeca-1(17),9,15,18-tetraen-17-yl 5-methylthienyl ketone (SI-8): IR (thin film) 3296, 2930, 2877, 1612, 1449, 1217, $761 \mathrm{~cm}^{-1} ;{ }^{1} \mathrm{H}$ NMR (500 MHz, DMSO-d6) $\delta 14.27$ (br.s, 1H, NH), 8.64 (br.s, 2H, C33-H, C34-H), 7.83 (d, 2H, J = 4.9 Hz, C32-H, C35-H), 7.41 (d, 1H, J = $8.3 \mathrm{~Hz}, \mathrm{C} 18-\mathrm{H}), 7.31$ (d, 1H, $J=8.3 \mathrm{~Hz}, \mathrm{C} 19-\mathrm{H}), 7.20$ (s, 1H, C16-H), 7.13 (d, 1H, J = 3.4 Hz, C22-H), $6.89(\mathrm{~d}, 1 \mathrm{H}, J=3.4 \mathrm{~Hz}, \mathrm{C} 23-\mathrm{H}), 4.89(\mathrm{~s}, 1 \mathrm{H}, \mathrm{C} 11-\mathrm{OH}), 4.78(\mathrm{~s}, 1 \mathrm{H}, \mathrm{C} 3-\mathrm{OH}), 4.14$ (d, 1H, J = 5.8 Hz, C9H), 3.66 (br.q, 1H, $J=6.8 \mathrm{~Hz}, \mathrm{C} 13-\mathrm{H}), 3.46$ (br.s, 1H, C28-H), 3.21 (br.s, 1H, C28-H), 3.20 (dd, 1H, $J=$ 13.2, $5.9 \mathrm{~Hz}, \mathrm{C} 14-\mathrm{H}), 3.15(\mathrm{t}, 1 \mathrm{H}, J=9.6 \mathrm{~Hz}, \mathrm{C} 2-\mathrm{H}), 2.47(\mathrm{dd}, 1 \mathrm{H}, J=13.2,7.8 \mathrm{~Hz}, \mathrm{C} 14-\mathrm{H}), 2.35(\mathrm{~s}, 3 \mathrm{H}$, C25- $\mathrm{H}_{3}$ ), 2.05-2.00 (m, 1H, C4-H), 1.90 (qd, 1H, J = 10.8, 3.4 Hz, C8-H), 1.86-1.68 (m, 5H, C3-H, C4$\mathrm{H}, \mathrm{C} 7-\mathrm{H}, \mathrm{C} 8-\mathrm{H}, \mathrm{C} 11-\mathrm{H}), 1.48$ (q, 1H, $J=10.3 \mathrm{~Hz}, \mathrm{C} 12-\mathrm{H}), 1.40$ (t, $1 \mathrm{H}, J=11.2 \mathrm{~Hz}, \mathrm{C} 7-\mathrm{H}), 1.25-1.16$ $(\mathrm{m}, 3 \mathrm{H}, \mathrm{C} 3-\mathrm{H}, \mathrm{C} 11-\mathrm{H}, \mathrm{C} 12-\mathrm{H}), 1.24$ (s, 3H, C27-H $), 0.87$ (s, 3H, C26-H ${ }_{3}$; ${ }^{13} \mathrm{C}$ NMR (125 MHz, DMSOd6) $\delta 190.21$ (C, C20), 159.19 (C, C31), 154.83 (C, C29), 150.79 (C, C24), 150.26 (CH, C33, C34), 142.74 (C, C21), 139.35 (C, C17), 138.37 (C, C1), 135.90 (CH, C22), 135.65 (C, C15), 131.93 (C, C10), 131.53 (CH, C19), 128.89 (CH, C18), 128.44 (CH, C16), 127.66 (CH, C23), 123.89 (CH, C9), 119.76 $(\mathrm{CH}, \mathrm{C} 32, \mathrm{C} 35), 84.41(\mathrm{C}, \mathrm{C} 5), 70.88(\mathrm{CH}, \mathrm{C} 13), 51.69(\mathrm{C}, \mathrm{C} 6), 47.62(\mathrm{CH}, \mathrm{C} 2), 43.61\left(\mathrm{CH}_{2}, \mathrm{C} 14\right)$, $41.11\left(\mathrm{CH}_{2}, \mathrm{C} 28\right), 35.56\left(\mathrm{CH}_{2}, \mathrm{C} 11\right), 33.29\left(\mathrm{CH}_{2}, \mathrm{C} 12\right), 31.54\left(\mathrm{CH}_{2}, \mathrm{C} 4\right), 30.74\left(\mathrm{CH}_{2}, \mathrm{C} 7\right), 27.51\left(\mathrm{CH}_{2}\right.$, C3), $24.03\left(\mathrm{CH}_{2}, \mathrm{C} 8\right), 15.56\left(\mathrm{CH}_{3}, \mathrm{C} 25\right), 14.85\left(\mathrm{CH}_{3}, \mathrm{C} 26\right), 14.57\left(\mathrm{CH}_{3}, \mathrm{C} 27\right)$; ESI-MS (+) $m / z 629$ $(\mathrm{M}+\mathrm{H})^{+}$. 


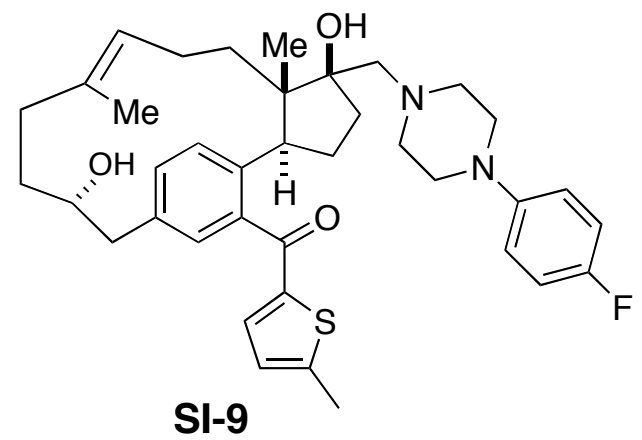

(2S,5S,6S,13S,9E)-5-[4-(4-Fluorophenyl)piperazin-1-ylmethyl]-5,13-dihydroxy-6,10-

dimethyltricyclo[13.2.2.0 $\left.{ }^{2,6}\right]$ nonadeca-1(17),9,15,18-tetraen-17-yl 5-methylthienyl ketone (SI-9): IR (thin film) 3430, 3296, 2947, 2875, 2828, 1633, $1510 \mathrm{~cm}^{-1} ;{ }^{1} \mathrm{H}$ NMR $\left(500 \mathrm{MHz}, \mathrm{CDCl}_{3}\right) \delta 7.39$ (d, $1 \mathrm{H}, J$ $=8.3 \mathrm{~Hz}, \mathrm{C} 18-\mathrm{H}), 7.30(\mathrm{~d}, 1 \mathrm{H}, J=8.3 \mathrm{~Hz}, \mathrm{C} 19-\mathrm{H}), 7.20(\mathrm{~s}, 1 \mathrm{H}, \mathrm{C} 16-\mathrm{H}), 7.14(\mathrm{~d}, 1 \mathrm{H}, J=3.4 \mathrm{~Hz}, \mathrm{C} 22-\mathrm{H})$, $6.96(\mathrm{t}, 2 \mathrm{H}, J=8.3 \mathrm{~Hz}, \mathrm{C} 35-\mathrm{H}, \mathrm{C} 37-\mathrm{H}), 6.86(\mathrm{dd}, 2 \mathrm{H}, J=8.8,4.4 \mathrm{~Hz}, \mathrm{C} 34-\mathrm{H}, \mathrm{C} 38-\mathrm{H}), 6.78(\mathrm{dd}, 1 \mathrm{H}, J=$ 3.4, $1.0 \mathrm{~Hz}, \mathrm{C} 23-\mathrm{H}), 4.45$ (br.s, $1 \mathrm{H}, \mathrm{C} 5-\mathrm{OH}), 4.14$ (br.d, $1 \mathrm{H}, J=5.4 \mathrm{~Hz}, \mathrm{C} 9-\mathrm{H}$ ), 3.89 (brq, $1 \mathrm{H}, J=6.8 \mathrm{~Hz}$, C13-H), 3.31 (dd, 1H, J = 13.2, 6.4 Hz, C14-H), 3.09 (br.s, 4H, C30-H , C31-H ), 2.94-2.91 (m, 1H, C2H), 2.83-2.79 (m, 2H, C29-H, C32-H), 2.71-2.67 (m, 2H, C29-H, C32-H), 2.67 (d, 1H, J = 13.7 Hz, C28$\mathrm{H}), 2.63(\mathrm{dd}, 1 \mathrm{H}, \mathrm{J}=13.2,8.3 \mathrm{~Hz}, \mathrm{C} 14-\mathrm{H}), 2.57\left(\mathrm{~s}, 3 \mathrm{H}, \mathrm{C} 25-\mathrm{H}_{3}\right), 2.18(\mathrm{~d}, 1 \mathrm{H}, J=13.2 \mathrm{~Hz}, \mathrm{C} 28-\mathrm{H})$, 2.00-1.92 (m, 3H, C3-H, C4-H, C11-H), 1.89-1.82 (m, 1H, C8-H), 1.82-1.76 (m, 3H, C3-H, C4-H, C8$\mathrm{H}), 1.70$ (s, 1H, C13-OH), 1.63-1.55 (m, 2H, C7-H, C12-H), 1.49 (d, 1H, J = 11.2 Hz, C7-H), 1.45-1.39 $(\mathrm{m}, 1 \mathrm{H}, \mathrm{C} 12-\mathrm{H}), 1.29\left(\mathrm{~s}, 3 \mathrm{H}, \mathrm{C} 27-\mathrm{H}_{3}\right), 1.29-1.26(\mathrm{~m}, 1 \mathrm{H}, \mathrm{C} 11-\mathrm{H}), 0.95\left(\mathrm{~s}, 3 \mathrm{H}, \mathrm{C} 26-\mathrm{H}_{3}\right) ;{ }^{13} \mathrm{C}$ NMR $(125$ $\left.\mathrm{MHz}, \mathrm{CDCl}_{3}\right) \delta 191.13(\mathrm{C}, \mathrm{C} 20), 157.44(\mathrm{C}, \mathrm{d}, J=240 \mathrm{~Hz}, \mathrm{C} 36), 151.12$ (C, C24), 147.99 (C, C33), 143.75 (C, C21), 140.13 (C, C17), 139.37 (C, C1), 135.98 (CH, C22), 135.12 (C, C15), 132.12 (C, C10), 131.85 (CH, C19), 128.93 (CH, C16), 128.79 (CH, C18), 127.05 (CH, C23), $124.94\left(\mathrm{CH}_{2}, \mathrm{C} 9\right), 118.05$ $(\mathrm{CH}, \mathrm{d}, J=6.9 \mathrm{~Hz}, \mathrm{C} 34, \mathrm{C} 38), 115.73(\mathrm{CH}, \mathrm{d}, J=22.1 \mathrm{~Hz}, \mathrm{C} 35, \mathrm{C} 37), 82.15$ (C, C5), 72.85 (CH, C13), $61.89\left(\mathrm{CH}_{2}, \mathrm{C} 28\right), 55.12\left(\mathrm{CH}_{2}, \mathrm{C} 29, \mathrm{C} 32\right), 50.88$ (C, C6), $50.64\left(\mathrm{CH}_{2}, \mathrm{C} 30, \mathrm{C} 31\right), 48.10(\mathrm{CH}, \mathrm{C} 2), 44.02$ $\left(\mathrm{CH}_{2}, \mathrm{C} 14\right), 35.80\left(\mathrm{CH}_{2}, \mathrm{C} 11\right), 35.61(\mathrm{C}, \mathrm{C} 4), 33.85\left(\mathrm{CH}_{2}, \mathrm{C} 12\right), 31.84\left(\mathrm{CH}_{2}, \mathrm{C} 7\right), 27.98\left(\mathrm{CH}_{2}, \mathrm{C} 3\right), 24.81$ $\left(\mathrm{CH}_{2}, \mathrm{C} 8\right), 16.40\left(\mathrm{CH}_{3}, \mathrm{C} 25\right), 14.97\left(\mathrm{CH}_{3}, \mathrm{C} 26\right), 14.85\left(\mathrm{CH}_{3}, \mathrm{C} 27\right)$; ESI-MS (+) $m / z 631(\mathrm{M}+\mathrm{H})^{+}$.

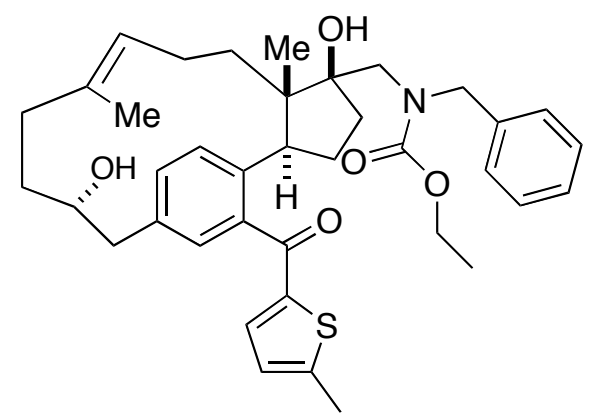

\section{SI-10}

$N$-Benzyl- $N$-[(2S,5S,6S,13S,9E)-5,13-dihydroxy-6,10-dimethyl-17-(5-methylthiophene-2-

carbonyl)tricyclo[13.2.2.0 $\left.{ }^{2,6}\right]$ nonadeca-1(17),9,15,18-tetraen-5-ylmethyl]carbamic acid ethyl ester (SI-10): IR (thin film) 3419, 2976, 2936, 2876, 1671, 1635, 1444, 1258, $732 \mathrm{~cm}^{-1}$; ${ }^{1} \mathrm{H}$ NMR $(500 \mathrm{MHz}$, $\left.\mathrm{CDCl}_{3}\right) \delta 7.39(\mathrm{~d}, 1 \mathrm{H}, J=7.8 \mathrm{~Hz}, \mathrm{C} 18-\mathrm{H}), 7.31-7.26(\mathrm{~m}, 4 \mathrm{H}, \mathrm{C} 19-\mathrm{H}, \mathrm{C} 32-\mathrm{H}, \mathrm{C} 33-\mathrm{H}, \mathrm{C} 34-\mathrm{H}), 7.20$ (s, $1 \mathrm{H}, \mathrm{C} 16-\mathrm{H}), 7.14(\mathrm{~d}, 1 \mathrm{H}, J=3.4 \mathrm{~Hz}, \mathrm{C} 22-\mathrm{H}), 7.13(\mathrm{~d}, 2 \mathrm{H}, J=6.8 \mathrm{~Hz}, \mathrm{C} 31-\mathrm{H}, \mathrm{C} 35-\mathrm{H}), 6.75(\mathrm{~d}, 1 \mathrm{H}, J=$ $3.4 \mathrm{~Hz}, \mathrm{C} 23-\mathrm{H}), 4.68(\mathrm{~d}, 1 \mathrm{H}, J=15.1 \mathrm{~Hz}, \mathrm{C} 29-\mathrm{H}), 4.23-4.14\left(\mathrm{~m}, 3 \mathrm{H}, \mathrm{C} 9-\mathrm{H}, \mathrm{C} 37-\mathrm{H}_{2}\right), 3.89(\mathrm{~d}, 1 \mathrm{H}, J=$ $15.1 \mathrm{~Hz}, \mathrm{C} 29-\mathrm{H}), 3.87$ (br.s, $1 \mathrm{H}, \mathrm{C} 13-\mathrm{H}), 3.68$ (d, $1 \mathrm{H}, J=15.1 \mathrm{~Hz}, \mathrm{C} 28-\mathrm{H}), 3.32$ (dd, $1 \mathrm{H}, J=13.2,6.5$ $\mathrm{Hz}, \mathrm{C} 14-\mathrm{H}), 3.10$ (dd, $1 \mathrm{H}, J=9.8,8.3 \mathrm{~Hz}, \mathrm{C} 2-\mathrm{H}), 3.01$ (d, 1H, $J=14.7 \mathrm{~Hz}, \mathrm{C} 28-\mathrm{H}), 2.59$ (dd, 1H, $J=$ 13.2, $7.8 \mathrm{~Hz}, \mathrm{C} 14-\mathrm{H}), 2.43$ (s, 3H, C25-H $), 1.98-1.72$ (m, 7H, C3-H, C4-H, C7-H, C8-H, C11-H), 1.69-1.55 (m, 2H, C3-H, C12-H), 1.42-1.37 (m, 2H, C7-H, C12-H), 1.30-1.25 (m, 1H, C11-H), 1.27 (s, $\left.3 \mathrm{H}, \mathrm{C} 27-\mathrm{H}_{3}\right), 1.23\left(\mathrm{t}, 3 \mathrm{H}, J=7.3 \mathrm{~Hz}, \mathrm{C} 38-\mathrm{H}_{3}\right), 0.95\left(\mathrm{~s}, 3 \mathrm{H}, \mathrm{C} 26-\mathrm{H}_{3}\right) ;{ }^{13} \mathrm{C} \mathrm{NMR}\left(125 \mathrm{MHz}, \mathrm{CDCl}_{3}\right)$

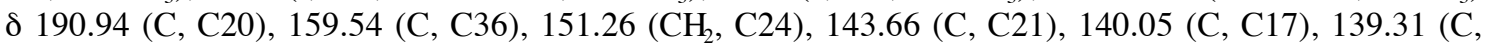
C1), 137.61 (C, C30), 136.00 (CH, C22), 135.16 (C, C15), 132.08 (C, C10), 131.83 (CH, C19), 128.91 
(CH, C18), 128.79 (CH, C16, C32, C34), 127.86 (CH, C31, C35), 127.67 (CH, C33), $126.99(\mathrm{CH}, \mathrm{C} 23)$, $124.95(\mathrm{CH}, \mathrm{C} 9), 86.76(\mathrm{C}, \mathrm{C} 5), 72.80(\mathrm{CH}, \mathrm{C} 13), 62.45\left(\mathrm{CH}_{2}, \mathrm{C} 37\right), 55.41\left(\mathrm{CH}_{2}, \mathrm{C} 28\right), 54.19\left(\mathrm{CH}_{2}\right.$, C29), 51.81 (C, C6), $48.21(\mathrm{CH}, \mathrm{C} 2), 44.11\left(\mathrm{CH}_{2}, \mathrm{C} 14\right), 35.96\left(\mathrm{CH}_{2}, \mathrm{C} 11\right), 33.98\left(\mathrm{CH}_{2}, \mathrm{C} 12\right), 31.26\left(\mathrm{CH}_{2}\right.$, C4), $30.75\left(\mathrm{CH}_{2}, \mathrm{C} 7\right), 28.33\left(\mathrm{CH}_{2}, \mathrm{C} 3\right), 24.47\left(\mathrm{CH}_{2}, \mathrm{C} 8\right), 16.23\left(\mathrm{CH}_{3}, \mathrm{C} 25\right), 14.67\left(\mathrm{CH}_{3}, \mathrm{C} 27, \mathrm{C} 38\right)$, $14.51\left(\mathrm{CH}_{3}, \mathrm{C} 26\right)$; ESI-MS (+) $\mathrm{m} / \mathrm{z} 630(\mathrm{M}+\mathrm{H})^{+}, 652(\mathrm{M}+\mathrm{Na})^{+}$.

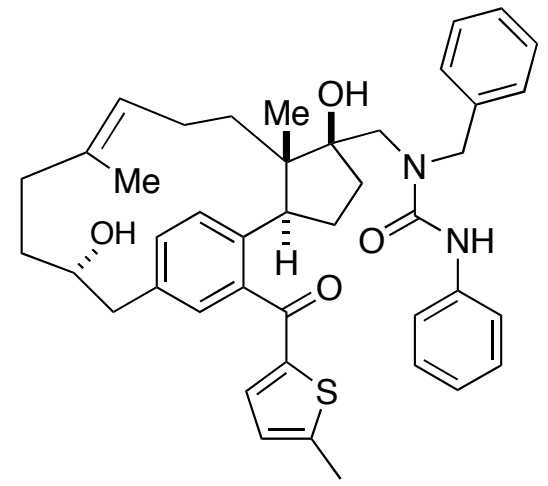

\section{$\mathrm{SI}-11$}

1-Benzyl-1-[(2S,5S,6S,13S,9E)-5,13-dihydroxy-6,10-dimethyl-17-(5-methylthiophene-2-

carbonyl)tricyclo[13.2.2.0 $\left.{ }^{2,6}\right]$ nonadeca-1(17),9,15,18-tetraen-5-ylmethyl]-3-phenylurea (SI-11) : IR (thin film) $3297,2928,1654,1622,1448,751 \mathrm{~cm}^{-1} ;{ }^{1} \mathrm{H}$ NMR $\left(500 \mathrm{MHz}, \mathrm{CDCl}_{3}\right) \delta$ 7.35-7.25 (m, 9H, C18-H, C19-H, C31-C35-H, C38-H, C42-H), 7.20 (t, 2H, J = 7.8 Hz, C39-H, C41-H), 7.19 (s, 1H, C16H), $7.14(\mathrm{~d}, 1 \mathrm{H}, J=3.4 \mathrm{~Hz}, \mathrm{C} 22-\mathrm{H}), 6.95(\mathrm{t}, 1 \mathrm{H}, J=7.3 \mathrm{~Hz}, \mathrm{C} 40-\mathrm{H}), 6.73(\mathrm{~d}, 1 \mathrm{H}, J=2.9 \mathrm{~Hz}, \mathrm{C} 23-\mathrm{H})$, $4.82(\mathrm{~d}, 1 \mathrm{H}, J=15.1 \mathrm{~Hz}, \mathrm{C} 29-\mathrm{H}), 4.18(\mathrm{~d}, 1 \mathrm{H}, J=7.8 \mathrm{~Hz}, \mathrm{C} 9-\mathrm{H}), 3.87-3.75(\mathrm{~m}, 3 \mathrm{H}, \mathrm{C} 13-\mathrm{H}, \mathrm{C} 28-\mathrm{H}$, C29-H), $3.30(\mathrm{dd}, 1 \mathrm{H}, J=13.2,6.4 \mathrm{~Hz}, \mathrm{C} 14-\mathrm{H}), 3.15(\mathrm{t}, 1 \mathrm{H}, J=10.2 \mathrm{~Hz}, \mathrm{C} 2-\mathrm{H}), 3.11(\mathrm{~d}, 1 \mathrm{H}, J=15.6$ $\mathrm{Hz}, \mathrm{C} 28-\mathrm{H}), 2.60(\mathrm{dd}, 1 \mathrm{H}, J=13.2,8.3 \mathrm{~Hz}, \mathrm{C} 14-\mathrm{H}), 2.38\left(\mathrm{~s}, 3 \mathrm{H}, \mathrm{C} 25-\mathrm{H}_{3}\right), 2.09-2.01(\mathrm{~m}, 1 \mathrm{H}, \mathrm{C} 4-\mathrm{H})$, 1.97-1.88 (m, 2H, C3-H, C11-H), 1.87-1.81 (m, 1H, C8-H), 1.78-1.72 (m, 3H, C4-H, C7-H, C8-H), 1.69-1.65 (m, 1H, C3-H), 1.62-1.55 (m, 1H, C12-H), 1.49-1.42 (m, 1H, C7-H), 1.37 (tdd, $1 \mathrm{H}, J=13.9$, 8.3, 2.4 Hz, C12-H), 1.27 (s, 3H, C27- $\left.\mathrm{H}_{3}\right), 1.25(\mathrm{dd}, 1 \mathrm{H}, J=15.1,8.8 \mathrm{~Hz}, \mathrm{C} 11-\mathrm{H}), 0.96\left(\mathrm{~s}, 3 \mathrm{H}, \mathrm{C} 26-\mathrm{H}_{3}\right)$; ${ }^{13} \mathrm{C}$ NMR (125 MHz, $\left.\mathrm{CDCl}_{3}\right) \delta 190.83$ (C, C20), 157.92 (C, C36), 151.51 (C, C24), 143.45 (C, C21), 140.07 (C, C17), 139.75 (C, C37), 138.69 (C, C1), 137.74 (C, C30), 136.02 (CH, C22), 135.45 (C, C15), 132.36 (C, C10), 131.90 (CH, C19), 129.06, 128.97, 128.88, 128.74 (CH x 4, C16, C18, C32, C34, C39, $\mathrm{C} 41), 127.88,127.83(\mathrm{CH} \times 2, \mathrm{C} 31, \mathrm{C} 33, \mathrm{C} 35), 127.02(\mathrm{CH}, \mathrm{C} 23), 124.58(\mathrm{CH}, \mathrm{C} 9), 122.67(\mathrm{CH}, \mathrm{C} 40)$, $119.56(\mathrm{CH}, \mathrm{C} 38, \mathrm{C} 42), 87.84(\mathrm{C}, \mathrm{C} 5), 72.69(\mathrm{CH}, \mathrm{C} 13), 55.41\left(\mathrm{CH}_{2}, \mathrm{C} 28\right), 54.04\left(\mathrm{CH}_{2}, \mathrm{C} 29\right), 51.77(\mathrm{C}$, C6), 48.14 (CH, C2), $44.04\left(\mathrm{CH}_{2}, \mathrm{C} 14\right), 35.88\left(\mathrm{CH}_{2}, \mathrm{C} 11\right), 33.87\left(\mathrm{CH}_{2}, \mathrm{C} 12\right), 31.35\left(\mathrm{CH}_{2}, \mathrm{C} 4\right), 30.34$ $\left(\mathrm{CH}_{2}, \mathrm{C} 7\right), 28.12\left(\mathrm{CH}_{2}, \mathrm{C} 3\right), 24.42\left(\mathrm{CH}_{2}, \mathrm{C} 8\right), 16.17\left(\mathrm{CH}_{3}, \mathrm{C} 25\right), 14.73\left(\mathrm{CH}_{3}, \mathrm{C} 27\right), 14.56\left(\mathrm{CH}_{3}, \mathrm{C} 26\right)$; ESI-MS (+) $m / z 677(\mathrm{M}+\mathrm{H})^{+}$.<smiles>C=C(C)CC[C@H](O)Cc1ccc(C2CCC(O)(CN(Cc3ccccc3)CC(O)COCc3ccccc3)C2(C)CCC=C(C)COCc2ccccc2)c(C(=O)c2ccc(C)s2)c1</smiles>

\section{SI-12}

$(2 S, 5 S, 6 S, 13 S, 9 E)$-5-[N-Benzyl- $N$-[3-benzyloxy-(2R)-2-hydroxypropyl]aminomethyl]-5,13dihydroxy-6,10-dimethyltricyclo[13.2.2. $\left.0^{2,6}\right]$ nonadeca-1(17),9,15,18-tetraen-17-yl 5-methylthienyl ketone (SI-12): IR (thin film) 3387, 2922, 2857, 1635, 1453, $735 \mathrm{~cm}^{-1} ;{ }^{1} \mathrm{H}$ NMR $\left(500 \mathrm{MHz}, \mathrm{CDCl}_{3}\right) \delta$ 
$7.34(\mathrm{~d}, 1 \mathrm{H}, J=8.3 \mathrm{~Hz}, \mathrm{C} 18-\mathrm{H}), 7.33$ (t, 2H, $J=7.3 \mathrm{~Hz}, \mathrm{C} 42-\mathrm{H}, \mathrm{C} 44-\mathrm{H}), 7.29-7.20$ (m, 9H, C19-H, C31-C35-H, C41-H, C43-H, C45-H), 7.18 (s, 1H, C16-H), 7.12 (d, 1H, J = 3.4 Hz, C22-H), 6.77 (d, $1 \mathrm{H}$, $J=3.4 \mathrm{~Hz}, \mathrm{C} 23-\mathrm{H}), 4.50$ (s, 2H, C39-H $), 4.16$ (d, 1H, $J=7.3 \mathrm{~Hz}, \mathrm{C} 9-\mathrm{H}), 3.95$ (br.s, 1H, C37-H), 3.86 $(\mathrm{dd}, 1 \mathrm{H}, J=14.2,7.3 \mathrm{~Hz}, \mathrm{C} 13-\mathrm{H}), 3.67(\mathrm{~d}, 1 \mathrm{H}, J=13.7 \mathrm{~Hz}, \mathrm{C} 29-\mathrm{H}), 3.61(\mathrm{~d}, 1 \mathrm{H}, J=12.7 \mathrm{~Hz}, \mathrm{C} 29-\mathrm{H})$, $3.43(\mathrm{dd}, 1 \mathrm{H}, J=9.8,3.4 \mathrm{~Hz}, \mathrm{C} 38-\mathrm{H}), 3.39$ (dd, $1 \mathrm{H}, J=9.8,5.9 \mathrm{~Hz}, \mathrm{C} 38-\mathrm{H}), 3.31$ (dd, $1 \mathrm{H}, J=13.2,6.4$ $\mathrm{Hz}, \mathrm{C} 14-\mathrm{H}), 2.95$ (dd, 1H, $J=10.3,8.3 \mathrm{~Hz}, \mathrm{C} 2-\mathrm{H}), 2.79$ (d, 1H, J = 13.7 Hz, C28-H), 2.63-2.57 (m, 3H,

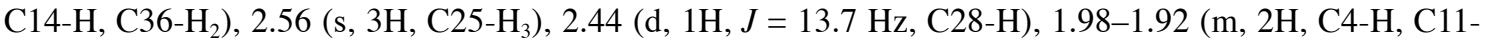
$\mathrm{H}), 1.85-1.76(\mathrm{~m}, 3 \mathrm{H}, \mathrm{C} 3-\mathrm{H}, \mathrm{C} 4-\mathrm{H}, \mathrm{C} 8-\mathrm{H}), 1.72-1.68$ (m, 1H, C8-H), 1.61-1.49 (m, 3H, C3-H, C7-H, C12-H), 1.41-1.32 (m, 2H, C7-H, C12-H), 1.27 (s, 3H, C27-H $), 1.27-1.24$ (m, 1H, C11-H), 0.91 (s, 3H, C26- $\left.\mathrm{H}_{3}\right) ;{ }^{13} \mathrm{C}$ NMR $\left(125 \mathrm{MHz}, \mathrm{CDCl}_{3}\right) \delta 191.12$ (C, C20), 150.97 (C, C24), 143.75 (C, C21), 140.10 (C, C17), 139.47 (C, C1), 138.88 (C, C30), 138.02 (C, C40), 135.94 (CH, C22), 135.07 (C, C15), 131.95 (C, C10), 131.77 (CH, C19), $129.25(\mathrm{CH}, \mathrm{C} 31, \mathrm{C} 35), 128.75,128.72(\mathrm{CH} x 2, \mathrm{C} 16, \mathrm{C} 18), 128.55(\mathrm{CH}, \mathrm{C} 32$, C34, C42, C44), 127.91 (CH, C41, C43, C45), 127.35 (CH, C33), 127.01 (CH, C23), 124.93 (CH, C9), $84.33(\mathrm{C}, \mathrm{C} 5), 73.56\left(\mathrm{CH}_{2}, \mathrm{C} 39\right), 72.75(\mathrm{CH}, \mathrm{C} 13), 72.71\left(\mathrm{CH}_{2}, \mathrm{C} 38\right), 68.51(\mathrm{CH}, \mathrm{C} 37), 62.40\left(\mathrm{CH}_{2}\right.$, C29), $59.24\left(\mathrm{CH}_{2}, \mathrm{C} 28\right), 58.91\left(\mathrm{CH}_{2}, \mathrm{C} 36\right), 51.19$ (C, C6), $48.13(\mathrm{CH}, \mathrm{C} 2), 44.03\left(\mathrm{CH}_{2}, \mathrm{C} 14\right), 35.88$ $\left(\mathrm{CH}_{2}, \mathrm{C} 11\right), 33.85\left(\mathrm{CH}_{2}, \mathrm{C} 12\right), 33.34\left(\mathrm{CH}_{2}, \mathrm{C} 4\right), 31.30\left(\mathrm{CH}_{2}, \mathrm{C} 7\right), 28.09\left(\mathrm{CH}_{2}, \mathrm{C} 3\right), 24.65\left(\mathrm{CH}_{2}, \mathrm{C} 8\right)$, $16.34\left(\mathrm{CH}_{3}, \mathrm{C} 25\right), 14.76\left(\mathrm{CH}_{3}, \mathrm{C} 26\right), 14.70\left(\mathrm{CH}_{3}, \mathrm{C} 27\right)$; ESI-MS (+) $\mathrm{m} / \mathrm{z}, 722(\mathrm{M}+\mathrm{H})^{+}$.

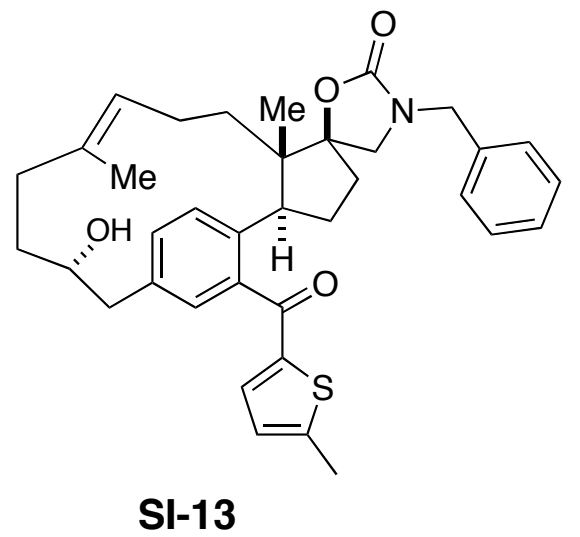

(5S)-3-Benzyloxazolidin-2-one-5-spiro-5'-(2'S,6' $S, 13$ ' $S, 9$ ' E)-6',10'-dimethyl-13'-hydroxy-17'-(5methylthiophene-2-carbonyl)tricyclo[13.2.2.0 $\left.{ }^{2}, 6^{\prime}\right]$ nonadeca-1'(17'),9',15',18'-tetraene (SI-13): IR (thin film) 3436, 2936, 1735, 1631, 1450, $730 \mathrm{~cm}^{-1} ;{ }^{1} \mathrm{H}$ NMR $\left(500 \mathrm{MHz}, \mathrm{CDCl}_{3}\right) \delta 7.36(\mathrm{~d}, 1 \mathrm{H}, J=8.3$ $\mathrm{Hz}, \mathrm{C} 18-\mathrm{H}), 7.34$ (t, 2H, $J=7.1 \mathrm{~Hz}, \mathrm{C} 33-\mathrm{H}, \mathrm{C} 35-\mathrm{H}), 7.31$ (d, 1H, J = 8.3 Hz, C19-H), 7.29 (t, $1 \mathrm{H}, J=7.3$ $\mathrm{Hz}, \mathrm{C} 34-\mathrm{H}), 7.25(\mathrm{~d}, 2 \mathrm{H}, J=6.8 \mathrm{~Hz}, \mathrm{C} 32-\mathrm{H}, \mathrm{C} 36-\mathrm{H}), 7.21(\mathrm{~d}, 1 \mathrm{H}, J=1.5 \mathrm{~Hz}, \mathrm{C} 16-\mathrm{H}), 7.10(\mathrm{~d}, 1 \mathrm{H}, J=$ $3.4 \mathrm{~Hz}, \mathrm{C} 22-\mathrm{H}), 6.75(\mathrm{dd}, 1 \mathrm{H}, J=3.9,1.0 \mathrm{~Hz}, \mathrm{C} 23-\mathrm{H}), 4.62(\mathrm{~d}, 1 \mathrm{H}, J=15.1 \mathrm{~Hz}, \mathrm{C} 30-\mathrm{H}), 4.17(\mathrm{~d}, 1 \mathrm{H}, J=$ $15.1 \mathrm{~Hz}, \mathrm{C} 30-\mathrm{H}), 3.97$ (br.s, $1 \mathrm{H}, \mathrm{C} 9-\mathrm{H}), 3.84$ (ddd, 1H, $J=14.2,8.3,2.4 \mathrm{~Hz}, \mathrm{C} 13-\mathrm{H}), 3.47$ (d, 1H, $J=8.8$ $\mathrm{Hz}, \mathrm{C} 29-\mathrm{H}), 3.28$ (dd, 1H, $J=13.2,6.4 \mathrm{~Hz}, \mathrm{C} 14-\mathrm{H}), 2.92$ (d, 1H, $J=8.8 \mathrm{~Hz}, \mathrm{C} 29-\mathrm{H}), 2.84(\mathrm{dd}, 1 \mathrm{H}, J=$

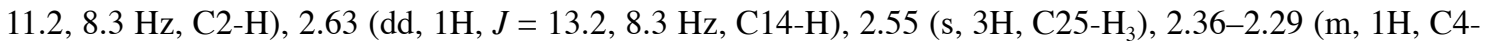
H), 2.06-1.95 (m, 1H, C3-H), 1.98-1.90 (m, 1H, C11-H), 1.84-1.73 (m, 4H, C3-H, C4-H, C8-H $), 1.60$ $(\mathrm{dt}, 1 \mathrm{H}, J=11.2,2.9 \mathrm{~Hz}, \mathrm{C} 7-\mathrm{H}), 1.57$ (td, $1 \mathrm{H}, J=10.3,2.4 \mathrm{~Hz}, \mathrm{C} 12-\mathrm{H}), 1.51$ (ddd, $1 \mathrm{H}, J=14.2,5.4,2.9$ $\mathrm{Hz}, \mathrm{C} 7-\mathrm{H}), 1.41$ (tdd, $1 \mathrm{H}, J=13.9,8.3,2.9 \mathrm{~Hz}, \mathrm{C} 12-\mathrm{H}), 1.29$ (s, 3H, C27-H $)_{3}, 1.26$ (ddd, $1 \mathrm{H}, J=15.1$, $8.3 \mathrm{~Hz}, 2.4 \mathrm{~Hz}, \mathrm{C} 11-\mathrm{H}), 1.01\left(\mathrm{~s}, 3 \mathrm{H}, \mathrm{C} 26-\mathrm{H}_{3}\right) ;{ }^{13} \mathrm{C} \mathrm{NMR}\left(125 \mathrm{MHz}, \mathrm{CDCl}_{3}\right) \delta 190.52(\mathrm{C}, \mathrm{C} 20), 158.00$ (C, C28), 151.49 (C, C24), 143.42 (C, C21), 139.86 (C, C17), 137.55 (C, C1), 136.23 (CH, C22), 136.04 (C, C31), 135.71 (C, C15), 132.77 (C, C10), 132.04 (CH, C19), 129.34 (CH, C16), 128.98 (CH, C33, C35), 128.63 (CH, C18), 128.11 (CH, C32, C36), 128.06 (CH, C34), 127.15 (CH, C23), 124.05 (CH, C9), 90.33 (C, C5), $72.87(\mathrm{CH}, \mathrm{C} 13), 52.79\left(\mathrm{CH}_{2}, \mathrm{C} 29\right), 50.00(\mathrm{C}, \mathrm{C} 6), 48.18\left(\mathrm{CH}_{2}, \mathrm{C} 30\right), 47.42(\mathrm{CH}, \mathrm{C} 2)$, $43.98\left(\mathrm{CH}_{2}, \mathrm{C} 14\right), 35.60\left(\mathrm{CH}_{2}, \mathrm{C} 11\right), 35.04\left(\mathrm{CH}_{2}, \mathrm{C} 4\right), 33.59\left(\mathrm{CH}_{2}, \mathrm{C} 12\right), 30.72\left(\mathrm{CH}_{2}, \mathrm{C} 7\right), 26.70\left(\mathrm{CH}_{2}\right.$, C3), $24.36\left(\mathrm{CH}_{2}, \mathrm{C} 8\right), 16.38\left(\mathrm{CH}_{3}, \mathrm{C} 25\right), 15.09\left(\mathrm{CH}_{3}, \mathrm{C} 27\right), 14.49\left(\mathrm{CH}_{3}, \mathrm{C} 26\right)$; ESI-MS (+) $m / z 584$ $(\mathrm{M}+\mathrm{H})^{+}$.

6) Building block selection

Building blocks were selected on the basis of satisfactory success ( $>70 \%$ purity by HPLC (photo diode array detector)) after cleavage of the compounds from the solid support with a chosen substrate in the all 
steps 
(6-1) Building blocks for first step (thiol)

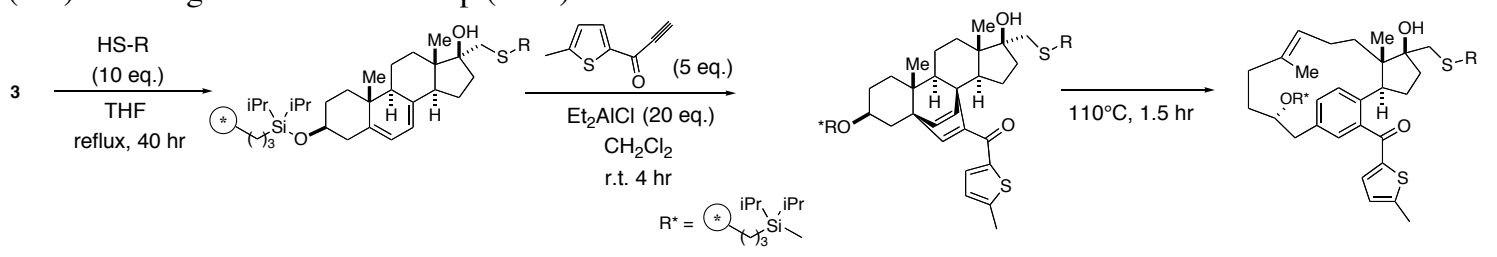

\begin{tabular}{|c|c|c|c|c|c|c|c|c|c|c|c|}
\hline No. & structure & MW & density & vendor & $\begin{array}{c}\text { first step } \\
\text { (epoxide opening) }\end{array}$ & $\begin{array}{l}\text { MW of } \\
\text { product }\end{array}$ & $\begin{array}{l}\text { third step } \\
\text { (Diels-Alder) }\end{array}$ & $\begin{array}{l}\text { MW of } \\
\text { product }\end{array}$ & $\begin{array}{c}\text { fourth step } \\
\text { (retro-Diels-Alder) }\end{array}$ & $\begin{array}{l}\text { MW of } \\
\text { product }\end{array}$ & select? \\
\hline 1 & & 110.18 & 1.073 & Ald. & $\underset{\text { messy }}{\mathrm{X}}$ & 410.62 & & 560.82 & & 560.82 & \\
\hline 2 & & 140.2 & 1.13 & Ald. & $\underset{\text { messy }}{\mathrm{x}}$ & 440.64 & & 590.84 & & 590.84 & \\
\hline 3 & & 101.13 & & Ald. & $\begin{array}{c}0 \\
90 \%\end{array}$ & 401.57 & $\begin{array}{c}\circ \\
90 \%\end{array}$ & 551.77 & $\begin{array}{c}0 \\
90 \%\end{array}$ & 551.77 & yes \\
\hline 4 & & 132.21 & & Ald. & $\underset{\text { no reaction }}{\mathrm{x}}$ & 432.65 & & 582.85 & & 582.85 & \\
\hline 5 & & 114.17 & & Ald. & $\begin{array}{c}0 \\
8098\end{array}$ & 414.61 & $\begin{array}{c}0 \\
8098\end{array}$ & 564.81 & $\begin{array}{c}0 \\
90 \%\end{array}$ & 564.81 & \\
\hline 6 & & 180.15 & & Ald. & $\begin{array}{c}\mathrm{X} \\
\text { no reaction }\end{array}$ & 480.59 & & 630.79 & & 630.79 & \\
\hline 7 & & 164.23 & & Avocado & $\begin{array}{c}0 \\
9098\end{array}$ & 464.67 & $\begin{array}{c}0 \\
80 \%\end{array}$ & 614.87 & $\begin{array}{c}0 \\
80 \%\end{array}$ & 614.87 & yes \\
\hline 8 & & 222.26 & & Avocado & $\underset{\text { no reaction }}{\mathrm{x}}$ & 522.7 & & 672.9 & & 672.9 & \\
\hline 9 & & 167.25 & & Ald. & $\begin{array}{c}0 \\
90 \% 6\end{array}$ & 467.69 & $\begin{array}{c}0 \\
80 \%\end{array}$ & 617.89 & $\begin{array}{c}0 \\
80 \%\end{array}$ & 617.89 & yes \\
\hline 10 & & 152.18 & & Fluka & $\frac{\mathrm{X}}{\text { no reaction }}$ & 452.62 & & 602.82 & & 602.82 & \\
\hline 11 & & 180.23 & & Ald. & $\begin{array}{c}0 \\
90 \%\end{array}$ & 480.67 & $\begin{array}{c}\circ \\
809 \%\end{array}$ & 630.87 & $\begin{array}{c}\circ \\
709 \%\end{array}$ & 630.87 & yes \\
\hline 12 & & 178.21 & & Ald. & $\begin{array}{c}0 \\
80 \%\end{array}$ & 478.65 & $\frac{\mathrm{X}}{\text { another compound }}$ & 628.85 & & 628.85 & \\
\hline 13 & & 178.21 & & Ald. & $\underset{\text { messy }}{\mathrm{x}}$ & 478.65 & & 628.85 & & 628.85 & \\
\hline 14 & & 178.22 & & Ald. & $\underset{50 \%}{x}$ & 478.66 & & 628.86 & & 628.86 & \\
\hline 15 & & 178.22 & & Ald. & $\begin{array}{c}0 \\
90 \% 8\end{array}$ & 478.66 & $\begin{array}{c}0 \\
80 \%\end{array}$ & 628.86 & $\begin{array}{c}0 \\
90 \%\end{array}$ & 628.86 & yes \\
\hline 16 & & 253.32 & & Ald. & $\underset{\text { messy }}{\mathrm{x}}$ & 553.76 & & 703.96 & & 703.96 & \\
\hline 17 & & 115.16 & & Ald. & $\underset{\text { messy }}{\mathrm{X}}$ & 415.6 & & 565.8 & & 565.8 & \\
\hline 18 & & 195.2 & & Ald. & $\begin{array}{c}\circ \\
90 \% \%\end{array}$ & 495.64 & $\begin{array}{c}\mathrm{x} \\
30 \%\end{array}$ & 645.84 & & 645.84 & \\
\hline
\end{tabular}




\begin{tabular}{|c|c|c|c|c|c|c|c|c|c|c|c|}
\hline No. & structure & MW & density & vendor & $\begin{array}{c}\text { first step } \\
\text { (epoxide opening) }\end{array}$ & $\begin{array}{l}\text { MW of } \\
\text { product }\end{array}$ & $\begin{array}{c}\text { third step } \\
\text { (Diels-Alder) }\end{array}$ & $\begin{array}{l}\text { MW of } \\
\text { product }\end{array}$ & $\begin{array}{c}\text { fourth step } \\
\text { (retro-Diels-Alder) }\end{array}$ & $\begin{array}{l}\text { MW of } \\
\text { product }\end{array}$ & select? \\
\hline 19 & & 151.19 & & Ald. & $\underset{\mathrm{X}}{\mathrm{X}}$ & 451.63 & & 601.83 & & 601.83 & \\
\hline 20 & & 112.15 & & Ald. & $\underset{\mathrm{X}}{\mathrm{X}}$ & 412.59 & & 562.79 & & 562.79 & \\
\hline 21 & & 179.2 & & Ald. & $\underset{\text { messy }}{\mathrm{X}}$ & 479.64 & & 629.84 & & 629.84 & \\
\hline 22 & & 252.34 & & Ald. & $\underset{\mathrm{x}}{\mathrm{X}}$ & 552.78 & & 702.98 & & 702.98 & \\
\hline 23 & & 211.31 & & Ald. & $\begin{array}{c}0 \\
90 \%\end{array}$ & 511.75 & $\begin{array}{c}0 \\
90 \%\end{array}$ & 661.95 & $\begin{array}{c}0 \\
90 \% 8\end{array}$ & 661.95 & yes \\
\hline 24 & & 152.18 & & Ald. & $\underset{\text { messy }}{\mathrm{x}}$ & 452.62 & & 602.82 & & 602.82 & \\
\hline 25 & & 127.17 & & Ald. & $\mathrm{X}$ & 427.61 & & 577.81 & & 577.81 & \\
\hline 26 & & 111.17 & & Ald. & $\underset{\text { messy }}{\mathrm{x}}$ & 411.61 & & 561.81 & & 561.81 & \\
\hline 27 & & 111.17 & & Ald. & $\underset{\text { messy }}{\mathrm{X}}$ & 411.61 & & 561.81 & & 561.81 & \\
\hline 28 & & 192.23 & & Fluka & $\underset{\mathrm{messy}}{\mathrm{x}}$ & 492.67 & & 642.87 & & 642.87 & \\
\hline 29 & & 284.29 & & Ald. & $\underset{\text { messy }}{\mathrm{x}}$ & 584.73 & & 734.93 & & 734.93 & \\
\hline
\end{tabular}

(6-2) Building blocks for first step (secondary amine)

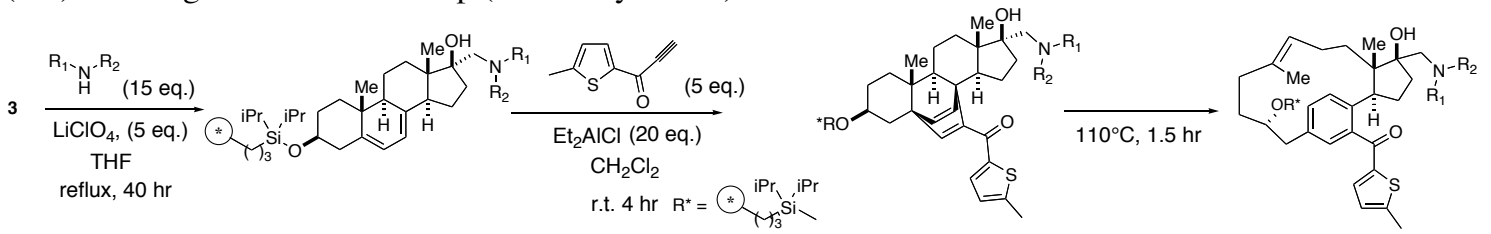

\begin{tabular}{|c|c|c|c|c|c|c|c|c|c|c|c|}
\hline No. & structure & MW & density & vendor & $\begin{array}{c}\text { first step } \\
\text { (epoxide opening) }\end{array}$ & $\begin{array}{l}\text { MW of } \\
\text { product }\end{array}$ & $\begin{array}{c}\text { thind step } \\
\text { (Diels-Alder) }\end{array}$ & $\begin{array}{l}\text { MW of } \\
\text { product }\end{array}$ & $\begin{array}{c}\text { fourth step } \\
\text { (retro-Diels-Alder) }\end{array}$ & $\begin{array}{l}\text { MW of } \\
\text { product }\end{array}$ & select? \\
\hline 1 & & 73.14 & 0.713 & Ald. & $\begin{array}{c}0 \\
90 \%\end{array}$ & 373.58 & $\begin{array}{c}0 \\
90 \%\end{array}$ & 523.78 & $\begin{array}{c}0 \\
90 \%\end{array}$ & 523.78 & yes \\
\hline 2 & & 75.11 & 0.935 & Ald. & $\begin{array}{c}0 \\
90 \%\end{array}$ & 375.55 & $\begin{array}{c}0 \\
90 \% 8\end{array}$ & 525.75 & $\begin{array}{c}0 \\
90 \% 8\end{array}$ & 525.75 & yes \\
\hline 3 & & 127.23 & 0.844 & Ald. & $\underset{\text { no reaction }}{\mathrm{X}}$ & 427.67 & & 577.87 & & 577.87 & \\
\hline 4 & & 128.22 & 0.882 & Ald. & $\underset{50 \%}{\mathrm{X}}$ & 428.66 & & 578.86 & & 578.86 & \\
\hline
\end{tabular}




\begin{tabular}{|c|c|c|c|c|c|c|c|c|c|c|c|}
\hline No. & structure & MW & density & vendor & $\begin{array}{c}\text { first step } \\
\text { (epoxide opening) }\end{array}$ & $\begin{array}{l}\text { MW of } \\
\text { product }\end{array}$ & $\begin{array}{c}\text { third step } \\
\text { (Diels-Alder) }\end{array}$ & $\begin{array}{l}\text { MW of } \\
\text { product }\end{array}$ & $\begin{array}{l}\text { fourth step } \\
\text { (retro-Diels-Alder) }\end{array}$ & $\begin{array}{l}\text { MW of } \\
\text { product }\end{array}$ & select? \\
\hline 5 & & 149.23 & 0.892 & Ald. & $\underset{\text { no reaction }}{\mathrm{X}}$ & 449.67 & & 599.87 & & 599.87 & \\
\hline 6 & & 136.19 & 0.979 & Ald. & $\underset{3095}{\mathrm{X}}$ & 436.63 & & 586.83 & & 586.83 & \\
\hline 7 & & 165.23 & 1.124 & Ald. & $\begin{array}{c}\mathrm{x} \\
3098\end{array}$ & 465.67 & & 615.87 & & 615.87 & \\
\hline 8 & & 219.32 & & Ald. & $\underset{\text { no reaction }}{\mathrm{X}}$ & 519.76 & & 669.96 & & 669.96 & \\
\hline 9 & & 101.15 & 1.025 & Add. & $\begin{array}{c}\circ \\
90 \%\end{array}$ & 401.59 & $\begin{array}{c}\circ \\
90 \% 8\end{array}$ & 551.79 & $\begin{array}{c}0 \\
9096\end{array}$ & 551.79 & yes \\
\hline 10 & & 154.25 & 0.946 & Ald. & $\underset{50 \%}{x}$ & 454.69 & & 604.89 & & 604.89 & \\
\hline 11 & & 253.34 & & Ald. & $\begin{array}{c}0 \\
80 \%\end{array}$ & 553.78 & $\begin{array}{c}0 \\
800 \%\end{array}$ & 703.98 & $\begin{array}{c}0 \\
90 \%\end{array}$ & 703.98 & yes \\
\hline 12 & & 85.15 & 0.86 & Ald. & $\begin{array}{c}0 \\
90 \% 8\end{array}$ & 385.59 & $\begin{array}{c}0 \\
90 \%\end{array}$ & 535.79 & $\begin{array}{c}0 \\
9098\end{array}$ & 535.79 & yes \\
\hline 13 & & 128.17 & & Arocado & $\begin{array}{c}\circ \\
90 \%\end{array}$ & 428.61 & $\begin{array}{c}\circ \\
90 \%\end{array}$ & 578.81 & $\begin{array}{c}\circ \\
90 \% 8\end{array}$ & 578.81 & yes \\
\hline 14 & & 154.25 & & Ald. & $\begin{array}{c}0 \\
90 \%\end{array}$ & 454.69 & $\underset{40 \%}{x}$ & 604.89 & & 604.89 & \\
\hline 15 & & 133.19 & 1.064 & Ald. & $\begin{array}{c}0 \\
8098\end{array}$ & 433.63 & $\begin{array}{c}\circ \\
90 \% 8\end{array}$ & 583.83 & $\begin{array}{c}0 \\
90 \% 8\end{array}$ & 583.83 & yes \\
\hline 16 & & 172.23 & & Ald. & $\begin{array}{c}\circ \\
80 \%\end{array}$ & 472.67 & $\stackrel{\circ}{80 \%}$ & 622.87 & $\begin{array}{c}\circ \\
80 \%\end{array}$ & 622.87 & yes \\
\hline 17 & & 175.27 & 0.997 & Ald. & $\begin{array}{c}0 \\
90 \%\end{array}$ & 475.71 & $\begin{array}{c}0 \\
90 \%\end{array}$ & 625.91 & $\begin{array}{c}0 \\
90 \%\end{array}$ & 625.91 & yes \\
\hline 18 & & 211.69 & & Ald. & $\begin{array}{c}\circ \\
80 \%\end{array}$ & 512.13 & $\begin{array}{c}\circ \\
80 \%\end{array}$ & 662.33 & $\begin{array}{c}0 \\
80 \% 8\end{array}$ & 662.33 & yes \\
\hline 19 & & 217.27 & & Ald. & $\begin{array}{c}\circ \\
80 \%\end{array}$ & 517.71 & $\begin{array}{c}\circ \\
70 \%\end{array}$ & 667.91 & $\begin{array}{c}\circ \\
9098\end{array}$ & 667.91 & yes \\
\hline 20 & & 231.29 & & Fluka & $\begin{array}{c}\circ \\
70 \%\end{array}$ & 531.73 & $\begin{array}{c}\circ \\
70 \%\end{array}$ & 681.93 & $\begin{array}{c}\circ \\
80 \%\end{array}$ & 681.93 & yes \\
\hline 21 & & 128.17 & & Ald. & $\begin{array}{c}0 \\
80 \% 8\end{array}$ & 428.61 & $\begin{array}{c}\circ \\
90 \% \%\end{array}$ & 578.81 & $\begin{array}{c}0 \\
90 \% 8\end{array}$ & 578.81 & yes \\
\hline 22 & & 130.19 & 1.061 & Ald. & $\begin{array}{c}\circ \\
90 \% 8\end{array}$ & 430.63 & $\begin{array}{c}\circ \\
90 \%\end{array}$ & 580.83 & $\begin{array}{c}\circ \\
80 \% 8\end{array}$ & 580.83 & yes \\
\hline 23 & & 180.22 & & Ald. & $\begin{array}{c}0 \\
90 \%\end{array}$ & 480.66 & $\begin{array}{c}0 \\
90 \% 8\end{array}$ & 630.86 & $\begin{array}{c}0 \\
90 \% 8\end{array}$ & 630.86 & yes \\
\hline 24 & & 26 & & Ald. & $\begin{array}{c}0 \\
80 \%\end{array}$ & 492.7 & $\begin{array}{c}\circ \\
80 \%\end{array}$ & 642.9 & $\begin{array}{c}0 \\
9098\end{array}$ & 642.9 & yes \\
\hline
\end{tabular}




\begin{tabular}{|c|c|c|c|c|c|c|c|c|c|c|c|}
\hline No. & structure & MW & density & vendor & $\begin{array}{c}\text { first step } \\
\text { (epoxide opening) }\end{array}$ & $\begin{array}{l}\text { MW of } \\
\text { product }\end{array}$ & $\begin{array}{c}\text { thind step } \\
\text { (Diels-Alder) }\end{array}$ & $\begin{array}{l}\text { MW of } \\
\text { product }\end{array}$ & $\begin{array}{c}\text { fouthth step } \\
\text { (retro-Diels-Alder) }\end{array}$ & $\begin{array}{l}\text { MW of } \\
\text { product }\end{array}$ & select? \\
\hline 25 & & 178.23 & & Arocado & $\begin{array}{c}0 \\
8098\end{array}$ & 478.67 & $\begin{array}{c}0 \\
90 \% \%\end{array}$ & 628.87 & $\begin{array}{c}0 \\
90 \% \%\end{array}$ & 628.87 & yes \\
\hline 26 & & 163.22 & 1.072 & Ald. & $\begin{array}{c}0 \\
9098\end{array}$ & 463.66 & $\begin{array}{c}0 \\
809 \%\end{array}$ & 613.86 & $\begin{array}{c}0 \\
908 \%\end{array}$ & 613.86 & yes \\
\hline 27 & & 220.27 & & Ald. & $\begin{array}{l}0 \\
80 \%\end{array}$ & 520.71 & $\underset{20 \%}{x}$ & 670.91 & & 670.91 & \\
\hline 28 & & 286.8 & & Ald. & $\begin{array}{c}0 \\
70 \%\end{array}$ & 587.24 & $\begin{array}{c}0 \\
70 \%\end{array}$ & 737.44 & $\begin{array}{c}0 \\
80 \% \%\end{array}$ & 737.44 & yes \\
\hline 29 & & 180.2 & & Lancaster & $\begin{array}{c}0 \\
7098\end{array}$ & 480.64 & $\begin{array}{c}0 \\
9098\end{array}$ & 630.84 & $\begin{array}{c}0 \\
90 \% \%\end{array}$ & 630.84 & yes \\
\hline 30 & & 220.27 & 1.142 & Ald. & $\begin{array}{c}0 \\
7098\end{array}$ & 520.71 & $\underset{\text { another compound }}{\mathrm{x}}$ & 670.91 & & 670.91 & \\
\hline 31 & & 202.3 & & Ald. & $\begin{array}{c}\circ \\
80 \%\end{array}$ & 502.74 & $\underset{50 \%}{x}$ & 652.94 & & 652.94 & \\
\hline 32 & & 87.12 & 0.999 & Ald. & $\begin{array}{c}0 \\
80 \%\end{array}$ & 387.56 & $\begin{array}{c}0 \\
90 \% 8\end{array}$ & 537.76 & $\begin{array}{c}0 \\
90 \%\end{array}$ & 537.76 & yes \\
\hline 33 & & 114.19 & 0.918 & Ald. & $\begin{array}{c}0 \\
909 \%\end{array}$ & 414.63 & $\underset{40 \% 8}{x}$ & 564.83 & & 564.83 & \\
\hline 34 & & 211.3 & 1.01 & Ald. & $\underset{\text { no reaction }}{x}$ & 511.74 & & 661.94 & & 661.94 & \\
\hline 35 & & 175.23 & & Fluka & $\underset{\text { no reaction }}{x}$ & 475.67 & & 625.87 & & 625.87 & \\
\hline 36 & & 219.28 & & Ald. & $\underset{\text { no reaction }}{\mathrm{X}}$ & 519.72 & & 669.92 & & 669.92 & \\
\hline 37 & & 263.33 & & Fluka & $\underset{\text { no reaction }}{\mathrm{X}}$ & 563.77 & & 713.97 & & 713.97 & \\
\hline 38 & & 185.36 & 0.795 & Ald. & $\underset{\text { no reaction }}{\mathrm{x}}$ & 485.8 & & 636 & & 636 & \\
\hline 39 & & 183.21 & & Ald. & $\underset{\text { no reaction }}{\mathrm{x}}$ & 483.65 & & 633.85 & & 633.85 & \\
\hline 40 & & 113.2 & 0.868 & Ald. & $\underset{\text { no reaction }}{x}$ & 413.64 & & 563.84 & & 563.84 & \\
\hline 41 & & 135.21 & 0.93 & Ald. & $\begin{array}{c}0 \\
7098\end{array}$ & 435.65 & $\begin{array}{c}0 \\
80 \%\end{array}$ & 585.85 & $\begin{array}{c}0 \\
80 \%\end{array}$ & 585.85 & yes \\
\hline 42 & & 117.15 & 1.029 & Ald. & $\underset{50 \%}{x}$ & 417.59 & & 567.79 & & 567.79 & \\
\hline 43 & & 221.31 & & Ald. & $\begin{array}{c}\circ \\
7098\end{array}$ & 521.75 & $\begin{array}{c}x \\
40 \%\end{array}$ & 671.95 & & 671.95 & \\
\hline 44 & & 136.2 & 0.984 & Ald. & $\begin{array}{c}\circ \\
7098\end{array}$ & 436.64 & $\begin{array}{c}0 \\
80 \%\end{array}$ & 586.84 & $\begin{array}{c}0 \\
70 \% 8\end{array}$ & 586.84 & \\
\hline
\end{tabular}




\begin{tabular}{|c|c|c|c|c|c|c|c|c|c|c|c|}
\hline No. & structure & MW & density & vendor & $\begin{array}{c}\text { first step } \\
\text { (epoxide opening) }\end{array}$ & $\begin{array}{l}\text { MW of } \\
\text { product }\end{array}$ & $\begin{array}{l}\text { third step } \\
\text { (Diels-Alder) }\end{array}$ & $\begin{array}{l}\text { MW of } \\
\text { product }\end{array}$ & $\begin{array}{c}\text { fourth step } \\
\text { (retro-Diels-Alder) }\end{array}$ & $\begin{array}{l}\text { MW of } \\
\text { product }\end{array}$ & select? \\
\hline 45 & & 304.35 & & Ald. & $\begin{array}{c}\mathrm{X} \\
\text { messy }\end{array}$ & 488.72 & & 638.92 & & 638.92 & \\
\hline 46 & & 139.2 & & Ald. & $\begin{array}{c}0 \\
8098\end{array}$ & 439.64 & $\begin{array}{c}0 \\
70 \%\end{array}$ & 589.84 & $\begin{array}{c}0 \\
80 \%\end{array}$ & 589.84 & yes \\
\hline 47 & & 113.2 & 0.896 & Ald. & $\begin{array}{c}0 \\
7098\end{array}$ & 413.64 & $\begin{array}{c}0 \\
90 \% \%\end{array}$ & 563.84 & $\begin{array}{c}0 \\
80 \%\end{array}$ & 563.84 & yes \\
\hline 48 & & 119.17 & 1.05 & Ald. & $\begin{array}{c}0 \\
70 \%\end{array}$ & 419.61 & $\begin{array}{c}0 \\
90 \% 8\end{array}$ & 569.81 & $\begin{array}{c}0 \\
90 \%\end{array}$ & 569.81 & yes \\
\hline
\end{tabular}

(6-3) Building blocks for first step (primary amine)
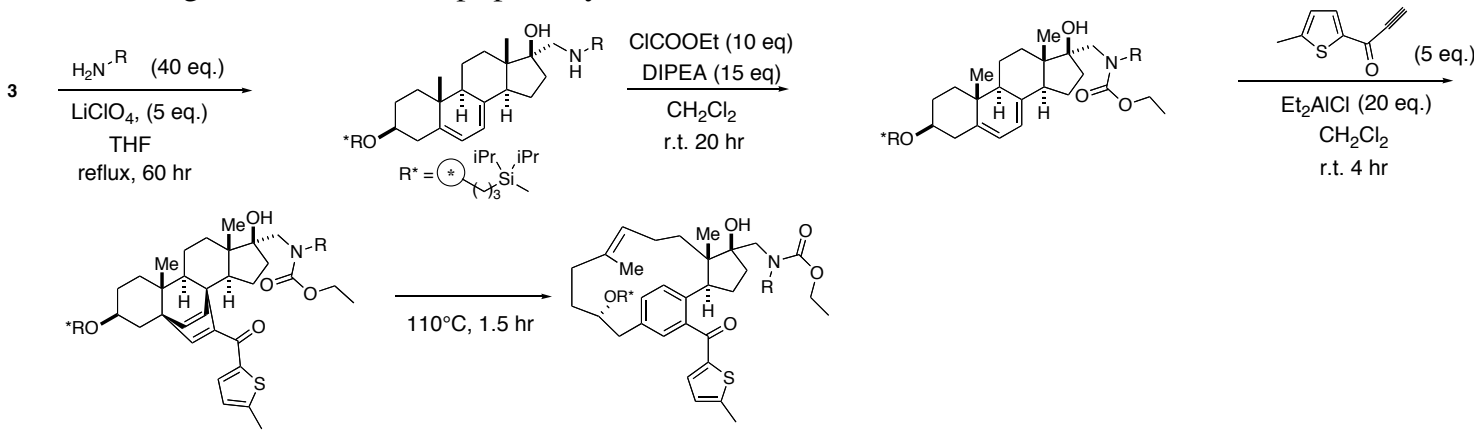

\begin{tabular}{|c|c|c|c|c|c|c|c|c|c|c|c|c|c|}
\hline No. & structure & MW & density & vendor & $\begin{array}{c}\text { first step } \\
\text { (epoxide opening) }\end{array}$ & $\begin{array}{l}\text { MW of } \\
\text { product }\end{array}$ & $\begin{array}{l}\text { second step } \\
\text { (acylation) }\end{array}$ & $\begin{array}{l}\text { MW of } \\
\text { product }\end{array}$ & $\begin{array}{l}\text { thind step } \\
\text { (Diels-Alder) }\end{array}$ & $\begin{array}{l}\text { MW of } \\
\text { product }\end{array}$ & $\begin{array}{c}\text { fourth step } \\
\text { (retro-Diels-Alder) }\end{array}$ & $\begin{array}{l}\text { MW of } \\
\text { product }\end{array}$ & select? \\
\hline 1 & & 59.11 & 0.719 & Aid. & $\begin{array}{c}0 \\
90 \% 8\end{array}$ & 359.55 & $\begin{array}{c}0 \\
90 \%\end{array}$ & 431.61 & $\begin{array}{c}0 \\
90 \% 8\end{array}$ & 581.81 & $\begin{array}{c}0 \\
90 \%\end{array}$ & 581.81 & yes \\
\hline 2 & & 89.14 & 0.874 & Aid. & $\begin{array}{c}0 \\
90 \%\end{array}$ & 389.58 & $\begin{array}{c}0 \\
9098\end{array}$ & 461.64 & $\begin{array}{c}0 \\
909 \%\end{array}$ & 611.84 & $\begin{array}{c}0 \\
90 \%\end{array}$ & 611.84 & yes \\
\hline 3 & & 99.17 & 0.867 & Aid. & $\begin{array}{c}0 \\
9098\end{array}$ & 399.61 & $\begin{array}{c}0 \\
90 \%\end{array}$ & 471.67 & $\begin{array}{c}0 \\
90 \%\end{array}$ & 621.87 & $\begin{array}{c}0 \\
9098\end{array}$ & 621.87 & \\
\hline 4 & & 165.28 & 0.933 & Aid. & $\begin{array}{c}\circ \\
90 \% 8\end{array}$ & 465.72 & $\begin{array}{c}0 \\
90 \%\end{array}$ & 537.78 & $\begin{array}{c}0 \\
909 \%\end{array}$ & 687.98 & $\begin{array}{c}0 \\
9098\end{array}$ & 687.98 & yes \\
\hline 5 & & 153.26 & 0.915 & Aid. & $\begin{array}{c}0 \\
9098\end{array}$ & 453.7 & $\begin{array}{c}\circ \\
90 \% 8\end{array}$ & 525.76 & $\begin{array}{c}\circ \\
9098\end{array}$ & 675.96 & $\begin{array}{c}\circ \\
90 \%\end{array}$ & 675.96 & yes \\
\hline 6 & & 101.15 & 0.98 & Aid. & $\begin{array}{c}\circ \\
90 \%\end{array}$ & 401.59 & $\begin{array}{c}0 \\
90 \% 8\end{array}$ & 473.65 & $\begin{array}{c}0 \\
809 \%\end{array}$ & 623.85 & $\begin{array}{c}\circ \\
8098\end{array}$ & 623.85 & yes \\
\hline 7 & & 130.19 & 0.992 & Aid. & $\begin{array}{c}\circ \\
9098\end{array}$ & 430.63 & $\begin{array}{c}\circ \\
90 \%\end{array}$ & 502.69 & $\begin{array}{c}0 \\
80 \%\end{array}$ & 652.89 & $\begin{array}{c}\circ \\
80 \%\end{array}$ & 652.89 & \\
\hline 8 & & 190.28 & 1.008 & Fluka & $\begin{array}{c}0 \\
90 \%\end{array}$ & 490.72 & $\begin{array}{c}\circ \\
90 \% 8\end{array}$ & 562.78 & $\begin{array}{c}0 \\
90 \%\end{array}$ & 712.98 & $\begin{array}{c}\circ \\
90 \%\end{array}$ & 712.98 & \\
\hline 9 & & 107.15 & 0.981 & Aid. & $\begin{array}{c}0 \\
90 \% 8\end{array}$ & 407.59 & $\begin{array}{c}\circ \\
9098\end{array}$ & 479.65 & $\begin{array}{c}0 \\
9098\end{array}$ & 629.85 & $\begin{array}{c}\circ \\
9098\end{array}$ & 629.85 & yes \\
\hline 10 & & 167.21 & 1.113 & Aid. & $\begin{array}{c}0 \\
900 \%\end{array}$ & 467.65 & $\begin{array}{c}\circ \\
9098\end{array}$ & 539.71 & $\begin{array}{c}0 \\
909 \%\end{array}$ & 689.91 & $\begin{array}{c}\circ \\
90 \%\end{array}$ & 689.91 & yes \\
\hline
\end{tabular}




\begin{tabular}{|c|c|c|c|c|c|c|c|c|c|c|c|c|c|}
\hline No. & structure & MW & density & vendor & $\begin{array}{c}\text { first step } \\
\text { (epoxide opening) }\end{array}$ & $\begin{array}{l}\text { MW of } \\
\text { product }\end{array}$ & $\begin{array}{l}\text { second step } \\
\text { (acylation) }\end{array}$ & $\begin{array}{l}\text { MW of } \\
\text { product }\end{array}$ & $\begin{array}{c}\text { third step } \\
\text { (Diels-Alder) }\end{array}$ & $\begin{array}{l}\text { MW of } \\
\text { product }\end{array}$ & $\begin{array}{c}\text { fourth step } \\
\text { (retro-Diels-Alder) }\end{array}$ & $\begin{array}{l}\text { MW of } \\
\text { product }\end{array}$ & select? \\
\hline 11 & & 191.15 & 1.252 & Aid. & $\stackrel{\circ}{90 \% 8}$ & 491.59 & $\stackrel{\circ}{90 \% 8}$ & 563.65 & $\underset{90 \% 8}{0}$ & 713.85 & $\begin{array}{c}0 \\
9098\end{array}$ & 713.85 & yes \\
\hline 12 & & 108.14 & 1.065 & Aid. & $\begin{array}{c}0 \\
9098\end{array}$ & 408.58 & $\underset{40 \%}{X}$ & 480.64 & & 630.84 & & 630.84 & \\
\hline 13 & & 133.19 & 1.038 & Aid. & $\begin{array}{c}0 \\
809 \%\end{array}$ & 433.63 & $\begin{array}{c}0 \\
8098\end{array}$ & 505.69 & $\begin{array}{c}0 \\
80 \%\end{array}$ & 655.89 & $\begin{array}{c}0 \\
8098\end{array}$ & 655.89 & \\
\hline 14 & & 157.21 & 1.073 & Aid. & $\begin{array}{c}0 \\
90 \% \%\end{array}$ & 457.65 & $\begin{array}{c}0 \\
80 \%\end{array}$ & 529.71 & $\begin{array}{c}0 \\
90 \% 8\end{array}$ & 679.91 & $\begin{array}{c}0 \\
80 \%\end{array}$ & 679.91 & yes \\
\hline 15 & & 127.21 & & Avocado & $\begin{array}{c}0 \\
90 \% 8\end{array}$ & 427.65 & $\begin{array}{c}0 \\
90 \% \%\end{array}$ & 499.71 & $\begin{array}{c}0 \\
90 \% 8\end{array}$ & 649.91 & $\begin{array}{c}\circ \\
90 \%\end{array}$ & 649.91 & yes \\
\hline 16 & & 160.22 & & Fluka & $\begin{array}{c}0 \\
90 \%\end{array}$ & 460.66 & $\begin{array}{c}0 \\
90 \%\end{array}$ & 532.72 & $\underset{\text { messy }}{\mathrm{x}}$ & 682.92 & & 682.92 & \\
\hline 17 & & 125.17 & 1.049 & Aid. & $\underset{\text { messy }}{\mathrm{x}}$ & 425.61 & & 497.67 & & 647.87 & & 647.87 & \\
\hline 18 & & 246.35 & & Fluka & $\underset{50 \% 8}{x}$ & 546.79 & & 618.85 & & 769.05 & & 769.05 & \\
\hline
\end{tabular}

(6-4) Building blocks for second step
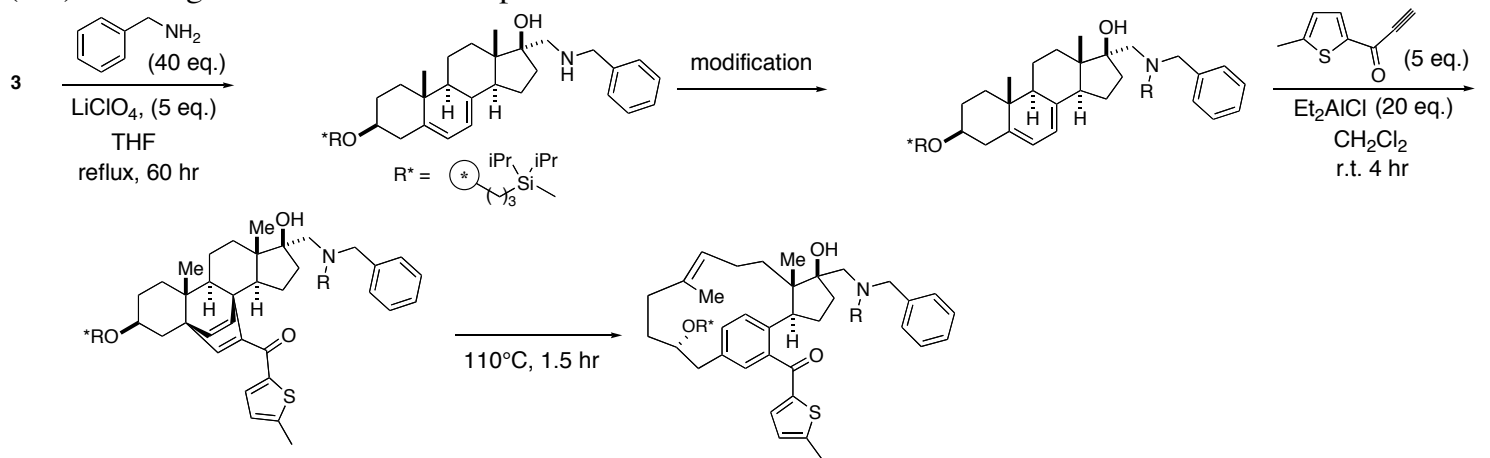

\begin{tabular}{|c|c|c|c|c|c|c|c|c|c|c|c|}
\hline No. & structure & MW & density & vendor & $\begin{array}{l}\text { second step } \\
\text { (modification) }\end{array}$ & $\begin{array}{l}\text { MW of } \\
\text { product }\end{array}$ & $\begin{array}{l}\text { third step } \\
\text { (Diels-Alder) }\end{array}$ & $\begin{array}{l}\text { MW of } \\
\text { product }\end{array}$ & $\begin{array}{c}\text { fourth step } \\
\text { (retro-Diels-Alder) }\end{array}$ & $\begin{array}{l}\text { MW of } \\
\text { product }\end{array}$ & select? \\
\hline 1 & & 108.52 & 1.187 & Ald. & $\begin{array}{c}0 \\
80 \%\end{array}$ & 479.65 & $\begin{array}{c}0 \\
80 \%\end{array}$ & 629.85 & $\begin{array}{c}0 \\
70 \%\end{array}$ & 629.85 & \\
\hline 2 & & 146.61 & 1.096 & Ald. & $\underset{80 \%}{0}$ & 517.74 & $\begin{array}{c}0 \\
8098\end{array}$ & 667.94 & $\begin{array}{c}0 \\
80 \%\end{array}$ & 667.94 & \\
\hline 3 & & 140.57 & 1.211 & Ald. & $\begin{array}{c}0 \\
9098\end{array}$ & 511.7 & $\begin{array}{c}0 \\
709 \%\end{array}$ & 661.9 & $\underset{6098}{x}$ & 661.9 & \\
\hline 4 & & 178.02 & & Ald. & $\begin{array}{c}0 \\
80 \%\end{array}$ & 512.69 & $\begin{array}{c}\circ \\
70 \%\end{array}$ & 662.89 & $\underset{6098}{x}$ & 662.89 & \\
\hline 5 & & 214.65 & & Ald. & $\begin{array}{c}0 \\
90 \%\end{array}$ & 585.78 & $\begin{array}{c}\circ \\
8098\end{array}$ & 735.98 & $\begin{array}{c}\circ \\
80 \%\end{array}$ & 735.98 & yes \\
\hline
\end{tabular}




\begin{tabular}{|c|c|c|c|c|c|c|c|c|c|c|c|}
\hline No. & stucture & мw & density & vendor & $\begin{array}{c}\text { second step } \\
\text { (modification) }\end{array}$ & $\begin{array}{l}\text { MW of } \\
\text { product }\end{array}$ & $\begin{array}{c}\text { thind step } \\
\text { (Diels-Alder) }\end{array}$ & $\begin{array}{l}\text { MW of } \\
\text { product }\end{array}$ & $\begin{array}{c}\text { fourth step } \\
\text { (retro-Diel-Alder) }\end{array}$ & $\begin{array}{l}\text { MW of } \\
\text { product }\end{array}$ & select? \\
\hline 6 & & 150.56 & 1.189 & Ald. & $\begin{array}{c}\circ \\
90 \% 8\end{array}$ & 521.69 & $\begin{array}{c}\circ \\
70 \% 8\end{array}$ & 671.89 & $\underset{50 \%}{x}$ & 671.89 & \\
\hline 7 & & 216.66 & & Ald. & $\begin{array}{c}\circ \\
900 \%\end{array}$ & 587.79 & $\begin{array}{c}\circ \\
80 \%\end{array}$ & 737.99 & $\begin{array}{l}0 \\
90 \%\end{array}$ & 737.99 & yes \\
\hline 8 & & 103.12 & & Ald. & $\begin{array}{c}\circ \\
80 \%\end{array}$ & 492.69 & $\begin{array}{c}\circ \\
70 \% 8\end{array}$ & 642.89 & $\begin{array}{c}X \\
40 \%\end{array}$ & 642.89 & \\
\hline 9 & & 218.21 & & Ald. & $\underset{\text { no reaction }}{x}$ & 607.78 & & 757.98 & & 757.98 & \\
\hline 10 & & 215.2 & & Ald. & $\underset{\text { no reaction }}{x}$ & 604.77 & & 754.97 & & 754.97 & \\
\hline 11 & & 234.2 & & Ald. & $\begin{array}{c}\circ \\
70 \% 8\end{array}$ & 623.77 & $\begin{array}{c}\circ \\
70 \%\end{array}$ & 773.97 & $\begin{array}{c}\circ \\
70 \%\end{array}$ & 773.97 & \\
\hline 12 & & 152.15 & & Ald. & $\begin{array}{c}0 \\
70 \%\end{array}$ & 541.72 & $\begin{array}{c}0 \\
70 \% 8\end{array}$ & 691.92 & $\begin{array}{c}0 \\
70 \%\end{array}$ & 691.92 & \\
\hline 13 & & 188.19 & & Ald. & $\begin{array}{c}\circ \\
7098\end{array}$ & 577.76 & $\begin{array}{c}\circ \\
70 \% 8\end{array}$ & 727.96 & $\begin{array}{c}\circ \\
7098\end{array}$ & 727.96 & \\
\hline 14 & & 244.31 & & Ald. & $\underset{\text { messy }}{\mathrm{x}}$ & 633.88 & & 784.08 & & 784.08 & \\
\hline 15 & & 85.11 & 0.866 & Ald. & $\begin{array}{c}0 \\
90 \% 8\end{array}$ & 492.7 & $\begin{array}{c}0 \\
90 \%\end{array}$ & 642.9 & $\begin{array}{c}0 \\
90 \%\end{array}$ & 642.9 & yes \\
\hline 16 & & 177.24 & & Ald. & $\begin{array}{l}\circ \\
900 \%\end{array}$ & 584.83 & $\begin{array}{l}\circ \\
90 \% \%\end{array}$ & 735.03 & $\begin{array}{l}0 \\
9008\end{array}$ & 735.03 & \\
\hline 17 & & 119.12 & 1.096 & Ald. & $\begin{array}{c}\circ \\
90 \%\end{array}$ & 526.71 & $\begin{array}{c}\circ \\
80 \% 8\end{array}$ & 676.91 & $\begin{array}{c}0 \\
90 \% 8\end{array}$ & 676.91 & yes \\
\hline 18 & & 149.15 & 1.151 & Ald. & $\begin{array}{c}0 \\
90 \%\end{array}$ & 556.74 & $\begin{array}{c}\circ \\
90 \%\end{array}$ & 706.94 & $\begin{array}{c}0 \\
9098\end{array}$ & 706.94 & yes \\
\hline 19 & & 147.17 & 1.045 & Ald. & $\begin{array}{c}0 \\
9098\end{array}$ & 554.76 & $\begin{array}{l}\circ \\
80 \%\end{array}$ & 704.96 & $\begin{array}{c}\circ \\
80 \%\end{array}$ & 704.96 & yes \\
\hline 20 & & 266.32 & & Fluka & $\begin{array}{c}\circ \\
70 \% \%\end{array}$ & 673.91 & $\begin{array}{c}\circ \\
80 \%\end{array}$ & 824.11 & $\begin{array}{l}0 \\
80 \%\end{array}$ & 824.11 & \\
\hline 21 & & 114.55 & 1.48 & Ald. & $\begin{array}{c}0 \\
80 \% 8\end{array}$ & 485.68 & $\begin{array}{c}0 \\
8098\end{array}$ & 635.88 & $\begin{array}{c}\circ \\
70 \%\end{array}$ & 635.88 & yes \\
\hline 22 & & 156.63 & 1.208 & Ald. & $\underset{\text { messy }}{\mathrm{x}}$ & 527.76 & & 677.96 & & 677.96 & \\
\hline 23 & & 256.06 & & Ald. & $\underset{\text { another compound }}{\mathrm{X}}$ & 627.19 & & 777.39 & & 777.39 & \\
\hline 24 & & 226.68 & & Ald. & $\begin{array}{c}0 \\
80 \%\end{array}$ & 597.81 & $\begin{array}{c}0 \\
80 \%\end{array}$ & 748.01 & $\begin{array}{c}\circ \\
90 \% 8\end{array}$ & 748.01 & \\
\hline 25 & & 182.65 & & Ald. & $\begin{array}{c}0 \\
80 \%\end{array}$ & 553.78 & $\begin{array}{l}\circ \\
90 \%\end{array}$ & 703.98 & $\begin{array}{c}\circ \\
90 \% 8\end{array}$ & 703.98 & yes \\
\hline 26 & & 190.65 & & Ald. & $\underset{60 \%}{x}$ & 561.78 & & 711.98 & & 711.98 & \\
\hline 27 & & 88.11 & 0.982 & Ald. & $\begin{array}{c}0 \\
70 \%\end{array}$ & 495.7 & $\begin{array}{c}0 \\
80 \%\end{array}$ & 645.9 & $\begin{array}{c}\circ \\
70 \%\end{array}$ & 645.9 & \\
\hline
\end{tabular}




\begin{tabular}{|c|c|c|c|c|c|c|c|c|c|c|c|}
\hline No. & structure & MW & density & vendor & $\begin{array}{c}\text { second step } \\
\text { (modification) }\end{array}$ & $\begin{array}{l}\text { MW of } \\
\text { product }\end{array}$ & $\begin{array}{l}\text { thind step } \\
\text { (Diels-Alder) }\end{array}$ & $\begin{array}{l}\text { MW of } \\
\text { product }\end{array}$ & $\begin{array}{c}\text { fourth step } \\
\text { (retro-Diels-Alder) }\end{array}$ & $\begin{array}{l}\text { MW of } \\
\text { product }\end{array}$ & select? \\
\hline 28 & & 74.08 & 1.117 & Ald. & $\begin{array}{c}0 \\
90 \% 8\end{array}$ & 481.67 & $\begin{array}{c}\circ \\
90 \%\end{array}$ & 631.87 & $\begin{array}{l}0 \\
80 \%\end{array}$ & 631.87 & yes \\
\hline 29 & & 144.17 & 1.018 & Ald. & $\underset{60 \%}{x}$ & 551.76 & & 701.96 & & 701.96 & \\
\hline 30 & & 120.15 & 1.051 & Ald. & $\begin{array}{c}0 \\
70 \%\end{array}$ & 527.74 & $\begin{array}{c}\circ \\
70 \%\end{array}$ & 677.94 & $\begin{array}{c}\circ \\
70 \% 8\end{array}$ & 677.94 & \\
\hline 31 & & 164.2 & 1.077 & Ald. & $\begin{array}{c}0 \\
90 \%\end{array}$ & 571.79 & $\begin{array}{c}0 \\
80 \%\end{array}$ & 721.99 & $\begin{array}{c}0 \\
70 \%\end{array}$ & 721.99 & yes \\
\hline 32 & & 108.52 & 1.135 & Ald. & $\begin{array}{c}\circ \\
90 \% 8\end{array}$ & 479.65 & $\begin{array}{c}\circ \\
90 \% 8\end{array}$ & 629.85 & $\begin{array}{c}\circ \\
90 \% 8\end{array}$ & 629.85 & yes \\
\hline 33 & & 170.59 & 1.195 & Ald. & $\begin{array}{l}\circ \\
80 \%\end{array}$ & 541.72 & $\begin{array}{c}x \\
50 \%\end{array}$ & 691.92 & & 691.92 & \\
\hline 34 & & 206.62 & 1.273 & TCI & $\begin{array}{c}\circ \\
70 \% 8\end{array}$ & 577.75 & $\begin{array}{c}\circ \\
80 \%\end{array}$ & 727.95 & $\begin{array}{c}\circ \\
70 \%\end{array}$ & 727.95 & yes \\
\hline 35 & & 218.73 & 1.02 & Ald. & $\begin{array}{c}0 \\
9098\end{array}$ & 589.86 & $\begin{array}{c}\circ \\
90 \% 8\end{array}$ & 740.06 & $\begin{array}{l}\circ \\
90 \%\end{array}$ & 740.06 & yes \\
\hline 36 & & 449.11 & & Ald. & $\underset{\text { messy }}{x}$ & 820.24 & & 970.44 & & 970.44 & \\
\hline 37 & carbonyl diimidazol & 162.15 & & Ald. & $\begin{array}{l}0 \\
90 \%\end{array}$ & 433.58 & $\begin{array}{c}\circ \\
70 \%\end{array}$ & 583.78 & $\begin{array}{c}\circ \\
70 \%\end{array}$ & 583.78 & yes \\
\hline 38 & oxalyl dimidazol & 190.16 & & Ald. & $\begin{array}{l}0 \\
90 \%\end{array}$ & 461.59 & $\underset{\text { another compound }}{\mathrm{x}}$ & 611.79 & $\begin{array}{c}\mathrm{X} \\
\text { another compound }\end{array}$ & 611.79 & \\
\hline
\end{tabular}

(6-5) Building blocks for third step
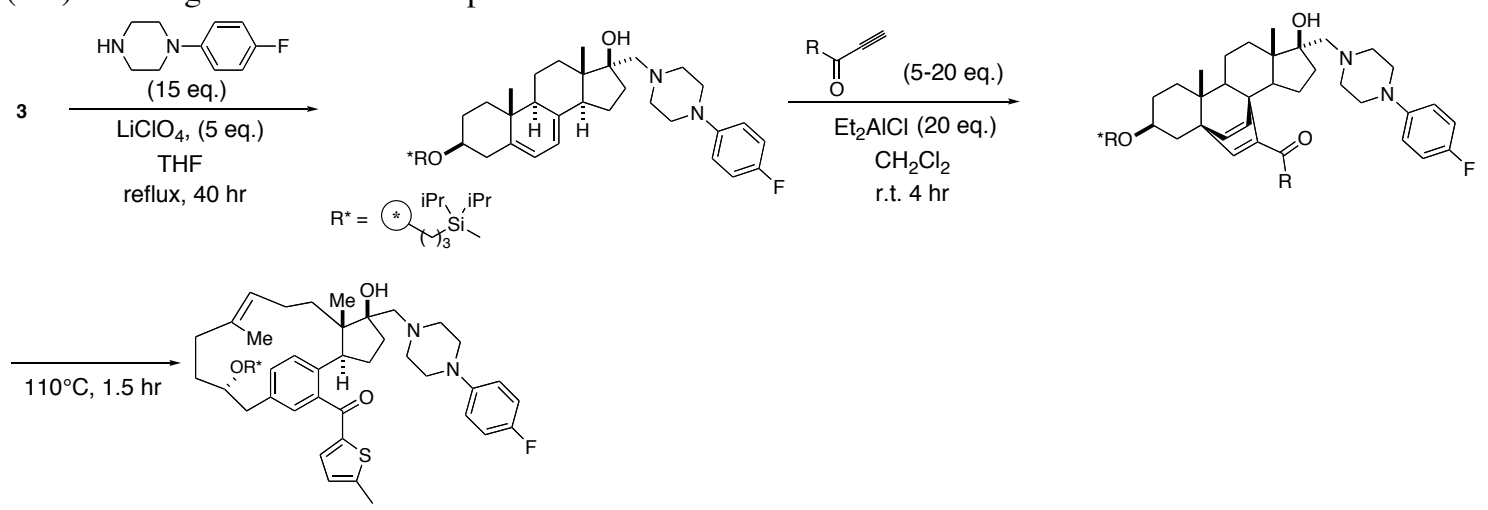

\begin{tabular}{|c|c|c|c|c|c|c|c|c|c|c|}
\hline No. & structure & $\begin{array}{l}\text { ynone } \\
\text { MW }\end{array}$ & note number & purity & $\begin{array}{l}\text { eq for } \\
\text { reaction }\end{array}$ & $\begin{array}{l}\text { third step } \\
\text { (Diels-Alder) }\end{array}$ & $\begin{array}{l}\text { MW of } \\
\text { product }\end{array}$ & $\begin{array}{l}\text { fourth step } \\
\text { (retro-Diels-Alder) }\end{array}$ & $\begin{array}{l}\text { MW of } \\
\text { product }\end{array}$ & select \\
\hline 1 & & 68.07 & $\begin{array}{l}\text { commercially } \\
\text { available (Aldi.) }\end{array}$ & 9998(NMR) & $20 \times 2$ & $\underset{40 \%}{x}$ & 548.73 & & 548.73 & \\
\hline 2 & & 108.14 & MK0245-49-1 & $\begin{array}{c}\text { 9098(LC) } \\
\text { 95\%(NMR) }\end{array}$ & 5 & $\underset{40 \%}{X}$ & 588.8 & & 588.8 & \\
\hline 3 & & 130.14 & MK0245-45-1 & $\begin{array}{c}100 \% 8(\mathrm{LC}) \\
100 \% \text { (NMR) }\end{array}$ & 5 & $\begin{array}{c}0 \\
80 \%\end{array}$ & 610.8 & $\begin{array}{c}0 \\
90 \%\end{array}$ & 610.8 & yes \\
\hline
\end{tabular}




\begin{tabular}{|c|c|c|c|c|c|c|c|c|c|c|}
\hline No. & structure & $\begin{array}{l}\text { ynone } \\
\text { MW }\end{array}$ & note number & purity & $\begin{array}{c}\text { eq for } \\
\text { reaction }\end{array}$ & $\begin{array}{c}\text { third step } \\
\text { (Diels-Alder) }\end{array}$ & $\begin{array}{l}\text { MW of } \\
\text { product }\end{array}$ & \begin{tabular}{|c} 
fourth step \\
(retro-Diels-Alder)
\end{tabular} & $\begin{array}{l}\text { MW of } \\
\text { product }\end{array}$ & select? \\
\hline 4 & & 166.12 & MK0245-68-1 & $\begin{array}{c}9598(\mathrm{LC}) \\
10008(\mathrm{NMR})\end{array}$ & 5 & $\begin{array}{c}0 \\
80 \%\end{array}$ & 646.78 & $\begin{array}{c}\circ \\
90 \%\end{array}$ & 646.78 & yes \\
\hline 5 & & 150.2 & MK0245-47-1 & $\begin{array}{l}\text { 9998(LC) } \\
\text { 9986(NMR) }\end{array}$ & 5 & $\begin{array}{c}\circ \\
90 \% 8\end{array}$ & 630.86 & $\begin{array}{c}\circ \\
9098\end{array}$ & 630.86 & yes \\
\hline 6 & & 186.23 & MK0245-48-1 & $\begin{array}{c}95 \% 8(\mathrm{LC}) \\
99 \%(\mathrm{NMR})\end{array}$ & 5 & $\begin{array}{c}0 \\
800 \%\end{array}$ & 666.89 & $\begin{array}{l}0 \\
80 \%\end{array}$ & 666.89 & yes \\
\hline 7 & & 183.21 & MK0245-50-1 & $\begin{array}{c}998 \%(\mathrm{LC}) \\
10098(\mathrm{NMR})\end{array}$ & 5 & $\underset{50 \% 8}{x}$ & 663.87 & & 663.87 & \\
\hline 8 & & 188.22 & MK0245-46-1 & $\begin{array}{c}9998(\mathrm{LC}) \\
100 \%(\mathrm{NMR})\end{array}$ & 5 & $\underset{40 \%}{x}$ & 668.88 & & 668.88 & \\
\hline 9 & & 198.14 & MK0245-78-1 & $\begin{array}{l}\text { 7008(LC) } \\
95 \%(\mathrm{MMR})\end{array}$ & 20 & $\begin{array}{c}0 \\
80 \%\end{array}$ & 678.8 & $\begin{array}{c}0 \\
8098\end{array}$ & 678.8 & yes \\
\hline 10 & & 136.19 & JAT4-8-dp & $\begin{array}{c}9098(\mathrm{LC}) \\
9998(\mathrm{MMR})\end{array}$ & 5 & $\begin{array}{c}0 \\
9098\end{array}$ & 616.85 & $\begin{array}{c}0 \\
9098\end{array}$ & 616.85 & yes \\
\hline 11 & & 124.18 & JAT4-5-dp & $\begin{array}{l}9098(\mathrm{LC}) \\
9998(\mathrm{NMR})\end{array}$ & 20 & $\underset{4098}{x}$ & 604.84 & & 604.84 & \\
\hline 12 & & 120.11 & MK0245-119-1 & $\begin{array}{c}100 \%(\mathrm{LC}) \\
100 \%(\mathrm{NMR})\end{array}$ & 5 & $\begin{array}{c}0 \\
80 \%\end{array}$ & 600.77 & $\begin{array}{c}\circ \\
70 \%\end{array}$ & 600.77 & yes \\
\hline 13 & & 160.17 & MK0245-106-1 & $\begin{array}{c}9998(\mathrm{LC}) \\
10098(\mathrm{NMR})\end{array}$ & 5 & $\begin{array}{c}\circ \\
9098\end{array}$ & 640.83 & $\begin{array}{c}\circ \\
909 \%\end{array}$ & 640.83 & yes \\
\hline 14 & & 124.14 & MK0245-107-1 & $\begin{array}{l}9998(\mathrm{LC}) \\
9998(\mathrm{NMR})\end{array}$ & 20 & $\underset{20 \%}{x}$ & 604.8 & & 604.8 & \\
\hline 15 & & 206.24 & MK0245-108-1 & $\begin{array}{c}100 \% 8(\mathrm{LC}) \\
100 \%(\mathrm{NMR})\end{array}$ & 5 & $\begin{array}{c}\circ \\
90 \%\end{array}$ & 686.9 & $\begin{array}{c}\circ \\
90 \%\end{array}$ & 686.9 & yes \\
\hline 16 & & 298.64 & MK0245-109-1 & $\begin{array}{c}100 \%(\mathrm{LC}) \\
100 \%(\mathrm{NMR})\end{array}$ & 20 & $\begin{array}{c}\circ \\
70 \%\end{array}$ & 779.3 & $\begin{array}{c}\circ \\
80 \%\end{array}$ & 779.3 & yes \\
\hline 17 & & 257.11 & MK0245-110-1 & $\begin{array}{c}5098(\mathrm{LC}) \\
95 \% \not(\mathrm{NMR})\end{array}$ & 20 & $\begin{array}{c}\circ \\
70 \%\end{array}$ & 737.77 & $\begin{array}{c}\circ \\
8098\end{array}$ & 737.77 & \\
\hline 18 & & 196.61 & MK0245-111-1 & $\begin{array}{c}9098(\mathrm{LC}) \\
100 \%(\mathrm{NMR})\end{array}$ & 20 & $\begin{array}{c}\circ \\
7008\end{array}$ & 677.27 & $\begin{array}{c}\circ \\
8096\end{array}$ & 677.27 & yes \\
\hline 19 & & 222.32 & MK0245-117-1 & $\begin{array}{c}900 \%(\mathrm{LC}) \\
9008(\mathrm{NMR})\end{array}$ & 5 & $\underset{\text { no reaction }}{x}$ & 702.98 & & 702.98 & \\
\hline 20 & & 206.24 & MK0245-118-1 & $\begin{array}{l}98 \% 8(\mathrm{LC}) \\
99 \%(\mathrm{NMR})\end{array}$ & 5 & $\begin{array}{c}\circ \\
9098\end{array}$ & 686.9 & $\begin{array}{c}0 \\
909 \%\end{array}$ & 686.9 & yes \\
\hline
\end{tabular}


7) Library realization and quality control

\section{(7-1) Epoxide opening (First step, synthesis of 4)}

3 (4.49 g, approximately 25650 beads) was divided into 31 portions of 150 beads and 10 portions of 2100 beads. 31 Portions of 150 beads were treated with thiols or secondary amines in THF by the optimized condition. On the other hand, 10 portions of 2100 beads were treated with primary amines. The detail conditions for each BB were shown in Table SI-1. After the reaction each portion of beads was washed, dried in vacuo, then subjected to tagging step. The binary encoding strategy was also shown in Table SI1. The structures of tags are shown in Figure SI-1.

Table SI-1

\begin{tabular}{|c|c|c|c|c|c|c|c|c|c|c|c|c|c|}
\hline BE-No. & stucture & MW & density & vendor & $\begin{array}{l}\text { No. of } \\
\text { beads }\end{array}$ & $\begin{array}{c}\text { amount for } \\
\text { reaction }\end{array}$ & other conditions & \begin{tabular}{|c|} 
T3B \\
C5C13 \\
\end{tabular} & \begin{tabular}{|c|} 
T4B \\
C6C13 \\
\end{tabular} & \begin{tabular}{|c|} 
T2A \\
C4C15 \\
\end{tabular} & $\begin{array}{l}\text { T3A } \\
\text { C5C15 } \\
\end{array}$ & \begin{tabular}{|c|} 
T4A \\
C6C15 \\
\end{tabular} & $\begin{array}{r}\text { T5A } \\
\text { C7C15 } \\
\end{array}$ \\
\hline BB 1-1 & & 101.13 & & Ald. & 150 & $45.5 \mathrm{mg}$ & $\begin{array}{c}\mathrm{THF}_{40.5 \mathrm{~mL}} \\
40 \mathrm{hr}\end{array}$ & & & & & & 1 \\
\hline BB1-2 & & 164.23 & & Arocado & 150 & $73.9 \mathrm{mg}$ & $\begin{array}{c}\text { THF } 1.5 \mathrm{~mL} \\
40 \mathrm{hr}\end{array}$ & & & & & 1 & 1 \\
\hline BB1-3 & & 167.25 & & Ald. & 150 & $75.3 \mathrm{mg}$ & $\begin{array}{l}\text { THF } 1.5 \mathrm{~mL} \\
40 \mathrm{hr}\end{array}$ & & & & 1 & & \\
\hline BB1-4 & & 180.23 & & Ald. & 150 & $81.1 \mathrm{mg}$ & $\begin{array}{l}\text { THF } 1.5 \mathrm{~mL} \\
40 \mathrm{hr}\end{array}$ & & & & 1 & & 1 \\
\hline BB1-5 & & 178.22 & & Ald. & 150 & $80.2 \mathrm{mg}$ & $\begin{array}{c}\text { THF } 1.5 \mathrm{~mL} \\
40 \mathrm{hr}\end{array}$ & & & & 1 & 1 & \\
\hline BB 1-6 & & 211.31 & & Ald. & 150 & $95.1 \mathrm{mg}$ & $\begin{array}{c}\mathrm{THF} 1.5 \mathrm{~mL} \\
40 \mathrm{hr}\end{array}$ & & & & 1 & 1 & 1 \\
\hline BB 1-7 & & 73.14 & 0.713 & Ald. & 150 & $\begin{array}{c}32.9 \mathrm{mg} \\
0.046 \mathrm{~mL}\end{array}$ & $\begin{array}{c}\text { THF } 1.5 \mathrm{~mL} \\
\text { LiClO4 } 16.0 \mathrm{mg} \\
40 \mathrm{hr}\end{array}$ & & & 1 & & & \\
\hline BB 1-8 & & 75.11 & 0.935 & Ald. & 150 & $\begin{array}{l}33.8 \mathrm{mg} \\
0.036 \mathrm{~mL}\end{array}$ & $\begin{array}{c}\text { THF } 1.5 \mathrm{~mL} \\
\text { LiC10416.0 mg } \\
40 \mathrm{hr}\end{array}$ & & & 1 & & & 1 \\
\hline BB 1-9 & & 101.15 & 1.025 & Ald. & 150 & $\begin{array}{l}45.5 \mathrm{mg} \\
0.044 \mathrm{~mL}\end{array}$ & $\begin{array}{c}\text { THF } 1.5 \mathrm{~mL} \\
\text { LiC104 } 16.0 \mathrm{mg} \\
40 \mathrm{hr}\end{array}$ & & & 1 & & 1 & \\
\hline BE1-10 & & 253.34 & & Ald. & 150 & $114 \mathrm{mg}$ & $\begin{array}{l}\text { THF } 1.5 \mathrm{~mL} \\
\text { LiClO4 } 46.0 \mathrm{mg} \\
40 \mathrm{hr}\end{array}$ & & & 1 & & 1 & 1 \\
\hline BE1-11 & & 85.15 & 0.86 & Ald. & 150 & $\begin{array}{r}38.3 \mathrm{mg} \\
0.044 \mathrm{~mL}\end{array}$ & $\begin{array}{c}\text { THF } 1.5 \mathrm{~mL} \\
\text { LiC104 } 16.0 \mathrm{mg} \\
40 \mathrm{hr}\end{array}$ & & & 1 & 1 & & \\
\hline BB1-12 & & 128.17 & & Avocado & 150 & $57.7 \mathrm{mg}$ & $\begin{array}{c}\text { THF } 1.5 \mathrm{~mL} \\
\text { LiClO4 } 46.0 \mathrm{mg} \\
40 \mathrm{hr}\end{array}$ & & & 1 & 1 & & 1 \\
\hline BB1-13 & & 133.19 & 1.064 & Ald. & 150 & $\begin{array}{c}59.9 \mathrm{mg} \\
0.056 \mathrm{~mL}\end{array}$ & $\begin{array}{l}\text { THF } 1.5 \mathrm{~mL} \\
\text { LiClO4 } 16.0 \mathrm{mg} \\
40 \mathrm{hr}\end{array}$ & & & 1 & 1 & 1 & \\
\hline
\end{tabular}




\begin{tabular}{|c|c|c|c|c|c|c|c|c|c|c|c|c|c|}
\hline BB-No. & structure & MW & density & vendor & $\begin{array}{c}\text { No. of } \\
\text { beads }\end{array}$ & $\begin{array}{c}\text { amount for } \\
\text { reaction }\end{array}$ & other conditions & \begin{tabular}{|l|} 
T3B \\
C5C13 \\
\end{tabular} & \begin{tabular}{|c|} 
TAB \\
C6C13 \\
\end{tabular} & \begin{tabular}{|l|} 
T2A \\
C4C15 \\
\end{tabular} & \begin{tabular}{|l|} 
T3A \\
C5C15 \\
\end{tabular} & \begin{tabular}{|l|} 
T4A \\
C6C15 \\
\end{tabular} & \begin{tabular}{|c|} 
TSA \\
C7C15 \\
\end{tabular} \\
\hline BB 1-14 & & 172.23 & & Ald. & 150 & $77.5 \mathrm{mg}$ & $\begin{array}{c}\text { THF } 1.5 \mathrm{~mL} \\
\mathrm{LiClO4} 4.0 \mathrm{mg} \\
40 \mathrm{hr}\end{array}$ & & & 1 & 1 & 1 & 1 \\
\hline BB1-15 & & 175.27 & 0.997 & Ald. & 150 & $\begin{array}{c}78.9 \mathrm{mg} \\
0.079 \mathrm{~mL}\end{array}$ & $\begin{array}{c}\text { THF } 1.5 \mathrm{~mL} \\
\text { LiClO4 } 16.0 \mathrm{mg} \\
40 \mathrm{hr}\end{array}$ & & 1 & & & & \\
\hline BB1-16 & & 211.69 & & Ald. & 150 & $95.3 \mathrm{mg}$ & $\begin{array}{c}\text { THF } 1.5 \mathrm{~mL} \\
\text { LiClO4 } 16.0 \mathrm{mg} \\
40 \mathrm{hr}\end{array}$ & & 1 & & & & 1 \\
\hline BB1-17 & & 217.27 & & Ald. & 150 & $97.8 \mathrm{mg}$ & $\begin{array}{c}\text { THF } 1.5 \mathrm{~mL} \\
\text { LiC10416.0 mg } \\
40 \mathrm{hr}\end{array}$ & & 1 & & & 1 & \\
\hline BB 1-18 & & 231.29 & & Fluka & 150 & $104 \mathrm{mg}$ & $\begin{array}{c}\text { THF } 1.5 \mathrm{~mL} \\
\text { LiC10416.0 } \mathrm{mg} \\
40 \mathrm{hr}\end{array}$ & & 1 & & & 1 & 1 \\
\hline BB 1-19 & & 128.17 & & Ald. & 150 & $57.7 \mathrm{mg}$ & $\begin{array}{c}\text { THF } 1.5 \mathrm{~mL} \\
\text { LiC104 } 16.0 \mathrm{mg} \\
40 \mathrm{hr}\end{array}$ & & 1 & & 1 & & \\
\hline BB1-20 & & 130.19 & 1.061 & Ald. & 150 & $\begin{array}{l}58.6 \mathrm{mg} \\
0.055 \mathrm{~mL}\end{array}$ & $\begin{array}{c}\text { THF } 1.5 \mathrm{~mL} \\
\text { LiC104.16.0 mg } \\
40 \mathrm{hr}\end{array}$ & & 1 & & 1 & & 1 \\
\hline BB1-21 & & 180.22 & & Ald. & 150 & $81.1 \mathrm{mg}$ & $\begin{array}{c}\text { THF } 1.5 \mathrm{~mL} \\
\text { LiC10416.0 mg } \\
40 \mathrm{hr}\end{array}$ & & 1 & & 1 & 1 & \\
\hline BB1-22 & & 192.26 & & Ald. & 150 & $86.5 \mathrm{mg}$ & $\begin{array}{l}\text { THF } 1.5 \mathrm{~mL} \\
\text { LiC10416.6 mg } \\
40 \mathrm{hr}\end{array}$ & & 1 & & 1 & 1 & 1 \\
\hline BB1-23 & & 178.23 & & Arocado & 150 & $80.2 \mathrm{mg}$ & $\begin{array}{c}\text { THF } 1.5 \mathrm{~mL} \\
\text { LiC10416.0 mg } \\
40 \mathrm{hr}\end{array}$ & & 1 & 1 & & & \\
\hline BB1-24 & & 163.22 & 1.072 & Ald. & 150 & $\begin{array}{c}73.5 \mathrm{mg} \\
0.068 \mathrm{~mL}\end{array}$ & $\begin{array}{c}\text { THF } 1.5 \mathrm{~mL} \\
\text { LiC104 } 16.0 \mathrm{mg} \\
40 \mathrm{hr}\end{array}$ & & 1 & 1 & & & 1 \\
\hline BB1-25 & & 286.8 & & Ald. & 150 & $129 \mathrm{mg}$ & $\begin{array}{c}\text { THF } 1.5 \mathrm{~mL} \\
\text { LiClO4 } 16.0 \mathrm{mg} \\
40 \mathrm{hr}\end{array}$ & & 1 & 1 & & 1 & \\
\hline BB1-26 & & 180.2 & & Lancaster & 150 & $81.1 \mathrm{mg}$ & $\begin{array}{c}\text { THF } 1.5 \mathrm{~mL} \\
\text { LiC10416.0 mg } \\
40 \mathrm{hr}\end{array}$ & & 1 & 1 & & 1 & 1 \\
\hline BB1-27 & & 87.12 & 0.999 & Ald. & 150 & $\begin{array}{l}39.2 \mathrm{mg} \\
0.039 \mathrm{~mL}\end{array}$ & $\begin{array}{c}\text { THF } 1.5 \mathrm{~mL} \\
\text { LiClO4 } 16.0 \mathrm{mg} \\
40 \mathrm{hr}\end{array}$ & & 1 & 1 & 1 & & \\
\hline BB1-28 & & 135.21 & 0.93 & Ald. & 150 & $\begin{array}{l}60.8 \mathrm{mg} \\
0.065 \mathrm{~mL}\end{array}$ & $\begin{array}{c}\text { THF } 1.5 \mathrm{~mL} \\
\text { LiC10416.0 mg } \\
40 \mathrm{hr}\end{array}$ & & 1 & 1 & 1 & & 1 \\
\hline BB1-29 & & 139.2 & & Ald. & 150 & $62.6 \mathrm{mg}$ & $\begin{array}{c}\text { THF } 1.5 \mathrm{~mL} \\
\text { LiC10416.0 mg } \\
40 \mathrm{hr}\end{array}$ & & 1 & 1 & 1 & 1 & \\
\hline BB1-30 & & 113.2 & 0.896 & Ald. & 150 & $\begin{array}{l}50.9 \mathrm{mg} \\
0.056 \mathrm{~mL}\end{array}$ & $\begin{array}{c}\text { THF } 1.5 \mathrm{~mL} \\
\text { LiClO4 } 16.0 \mathrm{mg} \\
40 \mathrm{hr}\end{array}$ & & 1 & 1 & 1 & 1 & 1 \\
\hline BE1-31 & & 119.17 & 1.05 & Ald. & 150 & $\begin{array}{l}53.6 \mathrm{mg} \\
0.051 \mathrm{~mL}\end{array}$ & $\begin{array}{l}\text { THF } 1.5 \mathrm{mI} \\
\text { LiClO4 } 16.6 \mathrm{mg} \\
40 \mathrm{hr}\end{array}$ & 1 & & & & & \\
\hline
\end{tabular}




\begin{tabular}{|c|c|c|c|c|c|c|c|c|c|c|c|c|c|}
\hline BB-No. & structure & Mw & density & vendor & $\begin{array}{l}\text { No. of } \\
\text { beads }\end{array}$ & $\begin{array}{l}\text { amount for } \\
\text { reaction }\end{array}$ & other conditions & \begin{tabular}{|c|}
$\mathrm{T} 3 \mathrm{~B}$ \\
$\mathrm{C5C13}$
\end{tabular} & \begin{tabular}{c|} 
T4B \\
C6C13
\end{tabular} & $\begin{array}{l}\text { T2A } \\
\text { C4C15 }\end{array}$ & \begin{tabular}{|l|} 
T3A \\
C5C15
\end{tabular} & $\begin{array}{l}\text { T4A } \\
\text { C6C15 }\end{array}$ & $\begin{array}{l}\text { T5A } \\
\text { C7C15 }\end{array}$ \\
\hline BB1-32 & & 59.11 & 0.719 & Aid. & 2100 & $\begin{array}{l}993 \mathrm{mg} \\
1.38 \mathrm{~mL}\end{array}$ & $\begin{array}{c}\text { THF } 20 \mathrm{~mL} \\
\text { LiC104223 mg } \\
60 \mathrm{hr}\end{array}$ & 1 & & & & & 1 \\
\hline BE1-33 & & 89.14 & 0.874 & Aid. & 2100 & $\begin{array}{c}1.50 \mathrm{~g} \\
1.72 \mathrm{~mL}\end{array}$ & $\begin{array}{c}\text { THF } 20 \mathrm{~mL} \\
\text { LiC104223 mg } \\
60 \mathrm{hr}\end{array}$ & 1 & & & & 1 & \\
\hline BB1-34 & & 165.28 & 0.933 & Aid. & 2100 & $\begin{array}{r}2.78 \mathrm{~g} \\
2.98 \mathrm{~mL}\end{array}$ & $\begin{array}{l}\text { THF } 20 \mathrm{~mL} \\
\text { LiC104223 mg } \\
60 \mathrm{hr}\end{array}$ & 1 & & & & 1 & 1 \\
\hline BB 1-35 & & 153.26 & 0.915 & Aid. & 2100 & $\begin{array}{r}2.57 \mathrm{~g} \\
2.81 \mathrm{~mL}\end{array}$ & $\begin{array}{c}\text { THF } 20 \mathrm{~mL} \\
\text { LiC104223 mg } \\
60 \mathrm{hr}\end{array}$ & 1 & & & 1 & & \\
\hline BE1-36 & & 101.15 & 0.98 & Aid. & 2100 & $\begin{array}{r}1.69 \mathrm{~g} \\
1.73 \mathrm{~mL}\end{array}$ & $\begin{array}{c}\text { THF } 20 \mathrm{~mL} \\
\text { LiC104223 mg } \\
60 \mathrm{hr}\end{array}$ & 1 & & & 1 & & 1 \\
\hline BB1-37 & & 107.15 & 0.981 & Aid. & 2100 & $\begin{array}{l}1.80 \mathrm{~g} \\
1.83 \mathrm{~mL}\end{array}$ & $\begin{array}{c}\text { THF } 20 \mathrm{~mL} \\
\text { LiC104223 mg } \\
60 \mathrm{hr}\end{array}$ & 1 & & & 1 & 1 & \\
\hline BE1-38 & & 167.21 & 1.113 & Aid. & 2100 & $\begin{array}{r}2.81 \mathrm{~g} \\
2.52 \mathrm{~mL}\end{array}$ & $\begin{array}{l}\text { THF } 20 \mathrm{~mL} \\
\text { LiC104 } 223 \mathrm{mg} \\
60 \mathrm{hr}\end{array}$ & 1 & & & 1 & 1 & 1 \\
\hline BB1-39 & & 191.15 & 1.252 & Aid. & 2100 & $\begin{array}{l}3.21 \mathrm{~g} \\
2.56 \mathrm{~mL}\end{array}$ & $\begin{array}{l}\text { THF } 20 \mathrm{~mL} \\
\text { LiC104223 mg } \\
60 \mathrm{hr}\end{array}$ & 1 & & 1 & & & \\
\hline BB1-40 & & 157.21 & 1.073 & Aid. & 2100 & $\begin{array}{c}2.64 \mathrm{~g} \\
2.46 \mathrm{~mL}\end{array}$ & $\begin{array}{c}\text { THF } 20 \mathrm{~mL} \\
\text { LiC104223 mg } \\
60 \mathrm{hr}\end{array}$ & 1 & & 1 & & & 1 \\
\hline BB1-41 & & 127.21 & & Avocado & 2100 & $2.14 \mathrm{~g}$ & $\begin{array}{c}\text { THF } 20 \mathrm{~mL} \\
\text { LiClO4 } 223 \mathrm{mg} \\
60 \mathrm{hr}\end{array}$ & 1 & & 1 & & 1 & \\
\hline
\end{tabular}

Figure SI-1. Structures of tags used in this paper 
<smiles>COc1cc(C(=O)C=N)ccc1OCC(C)COc1c(Cl)cc(Cl)cc1Cl</smiles>

T3B: $\mathrm{n}=3$

T4B: $\mathrm{n}=4$

T5B: $\mathrm{n}=5$<smiles>COc1cc(C(=O)C=N)ccc1OCCCOc1c(Cl)c(Cl)c(Cl)c(Cl)c1Cl</smiles>

T2A: $n=2 \quad$ T8A: $n=8$

T3A: $n=3 \quad$ T9A: $n=9$

T4A: $n=4 \quad$ T10A: $n=10$

T5A: $n=5 \quad$ T13A: $n=13$

T6A: $n=6 \quad$ T14A: $n=14$

T7A: $\mathrm{n}=7$ 


\section{(7-2) Quality check after the first step}

Three beads were selected from each portion $(3 \times 41=123$ beads $)$. The compounds on the beads were cleaved individually. While the compounds dissolved in $\mathrm{MeOH}(100 \mathrm{ml})$ were analyzed by LC-MS, Tags on the beads were cleaved and analyzed by GC. The results are shown in Table SI-2.

\begin{tabular}{|c|c|c|c|c|c|c|c|c|c|c|c|}
\hline Bead & \begin{tabular}{|c|} 
T3B \\
C5C13
\end{tabular} & \begin{tabular}{|l|} 
T4B \\
C6Cl3
\end{tabular} & $\begin{array}{l}\text { T2A } \\
\text { C4C15 }\end{array}$ & \begin{tabular}{|c|} 
T3A \\
C5C15
\end{tabular} & \begin{tabular}{|l|} 
T4A \\
C6C15
\end{tabular} & $\begin{array}{c}\text { T5A } \\
\text { C7C15 }\end{array}$ & $\begin{array}{c}\mathrm{BB} \\
\text { (expect) }\end{array}$ & $\begin{array}{c}\text { MW } \\
\text { (calcd) }\end{array}$ & \begin{tabular}{|c|} 
MW \\
(LC-MS)
\end{tabular} & \begin{tabular}{|c|} 
confirmed \\
$\mathrm{BB}$
\end{tabular} & $\begin{array}{l}\text { purity of } \\
\text { product }\end{array}$ \\
\hline BB1-1a & & & & & & 1 & 1 & 401 & 402 & 1 & 9098 \\
\hline b & & & & & & 1 & 1 & 401 & 402 & 1 & $90 \%$ \\
\hline c & & & & & & 1 & 1 & 401 & 402 & 1 & 9098 \\
\hline BB1-2a & & & & & 1 & 1 & 2 & 464 & 465 & 2 & $60 \%$ \\
\hline $\mathrm{b}$ & & & & & 1 & 1 & 2 & 464 & 465 & 2 & $60 \%$ \\
\hline $\mathrm{c}$ & & & & & 1 & 1 & 2 & 464 & 465 & 2 & $609 \%$ \\
\hline BE1-3a & & & & 1 & & & 3 & 467 & 468 & 3 & $70 \%$ \\
\hline $\mathrm{b}$ & & & & 1 & & & 3 & 467 & 468 & 3 & 7098 \\
\hline $\mathrm{c}$ & & & & 1 & & & 3 & 467 & 468 & 3 & 7096 \\
\hline BB1-4a & & & & 1 & & 1 & 4 & 480 & 481 & 4 & $80 \%$ \\
\hline $\mathrm{b}$ & & & & 1 & & 1 & 4 & 480 & 481 & 4 & 8095 \\
\hline $\mathrm{c}$ & & & & 1 & & 1 & 4 & 480 & 481 & 4 & $80 \%$ \\
\hline BB1-5a & & & & 1 & 1 & & 5 & 478 & 479 & 5 & $80 \%$ \\
\hline $\mathrm{b}$ & & & & 1 & 1 & & 5 & 478 & 479 & 5 & 8098 \\
\hline $\mathrm{c}$ & & & & 1 & 1 & & 5 & 478 & 479 & 5 & $80 \%$ \\
\hline BB1-6a & & & & 1 & 1 & 1 & 6 & 511 & 512 & 6 & 8096 \\
\hline b & & & & 1 & 1 & 1 & 6 & 511 & 512 & 6 & 8098 \\
\hline $\mathrm{c}$ & & & & 1 & 1 & 1 & 6 & 511 & 512 & 6 & $80 \%$ \\
\hline BB1-7a & & & 1 & & & & 7 & 374 & 375 & 7 & $90 \%$ \\
\hline b & & & 1 & & & & 7 & 374 & 375 & 7 & 9098 \\
\hline c & & & 1 & & & & 7 & 374 & 375 & 7 & $90 \%$ \\
\hline BB 1-8a & & & 1 & & & 1 & 8 & 375 & 376 & 8 & 9098 \\
\hline $\mathrm{b}$ & & & 1 & & & 1 & 8 & 375 & 376 & 8 & 9098 \\
\hline c & & & 1 & & & 1 & 8 & 375 & 376 & 8 & 9098 \\
\hline BB1-9a & & & 1 & & 1 & & 9 & 401 & 402 & 9 & $90 \%$ \\
\hline $\mathrm{b}$ & & & 1 & & 1 & & 9 & 401 & 402 & 9 & 9098 \\
\hline c & & & 1 & & 1 & & 9 & 401 & 402 & 9 & 9098 \\
\hline BB1-10a & & & 1 & & 1 & 1 & 10 & 553 & 538 & 10 & $80 \%$ \\
\hline $\mathrm{b}$ & & & 1 & & 1 & 1 & 10 & 553 & 538 & 10 & 8098 \\
\hline c & & & 1 & & 1 & 1 & 10 & 553 & 538 & 10 & 8096 \\
\hline BB1-11a & & & 1 & 1 & & & 11 & 385 & 386 & 11 & $909 \%$ \\
\hline $\mathrm{b}$ & & & 1 & 1 & & & 11 & 385 & 386 & 11 & $90 \%$ \\
\hline $\mathrm{c}$ & & & 1 & 1 & & & 11 & 385 & 386 & 11 & $90 \%$ \\
\hline BB1-12a & & & 1 & 1 & & 1 & 12 & 428 & 429 & 12 & $90 \% 8$ \\
\hline $\mathrm{b}$ & & & 1 & 1 & & 1 & 12 & 428 & 429 & 12 & $90 \%$ \\
\hline c & & & 1 & 1 & & 1 & 12 & 428 & 429 & 12 & $90 \%$ \\
\hline BB1-13a & & & 1 & 1 & 1 & & 13 & 433 & 434 & 13 & 9098 \\
\hline $\mathrm{b}$ & & & 1 & 1 & 1 & & 13 & 433 & 434 & 13 & $90 \%$ \\
\hline $\mathrm{c}$ & & & 1 & 1 & 1 & & 13 & 433 & 434 & 13 & $90 \%$ \\
\hline BB1-14a & & & 1 & 1 & 1 & 1 & 14 & 472 & 473 & 14 & $809 \%$ \\
\hline $\mathrm{b}$ & & & 1 & 1 & 1 & 1 & 14 & 472 & 473 & 14 & $80 \%$ \\
\hline $\mathrm{c}$ & & & 1 & 1 & 1 & 1 & 14 & 472 & 473 & 14 & $80 \%$ \\
\hline BB1-15a & & 1 & & & & & 15 & 475 & 476 & 15 & $90 \% 8$ \\
\hline $\mathrm{b}$ & & 1 & & & & & 15 & 475 & 476 & 15 & $90 \%$ \\
\hline $\mathrm{c}$ & & 1 & & & & & 15 & 475 & 476 & 15 & 9098 \\
\hline BB1-16a & & 1 & & & & 1 & 16 & 511 & 512 & 16 & $90 \%$ \\
\hline $\mathrm{b}$ & & 1 & & & & 1 & 16 & 511 & 512 & 16 & 9098 \\
\hline $\mathrm{c}$ & & 1 & & & & 1 & 16 & 511 & 512 & 16 & 9098 \\
\hline BB1-17a & & 1 & & & 1 & & 17 & 517 & 518 & 17 & $80 \%$ \\
\hline $\mathrm{b}$ & & 1 & & & 1 & & 17 & 517 & 518 & 17 & 8096 \\
\hline c & & 1 & & & 1 & & 17 & 517 & 518 & 17 & $80 \%$ \\
\hline BB1-18a & & 1 & & & 1 & 1 & 18 & 531 & 532 & 18 & 9098 \\
\hline $\mathrm{b}$ & & 1 & & & 1 & 1 & 18 & 531 & 532 & 18 & $90 \%$ \\
\hline c & & 1 & & & 1 & 1 & 18 & 531 & 532 & 18 & 9098 \\
\hline BB1-19a & & 1 & & 1 & & & 19 & 428 & 429 & 19 & 8098 \\
\hline $\mathrm{b}$ & & 1 & & 1 & & & 19 & 428 & 429 & 19 & 8098 \\
\hline $\mathrm{c}$ & & 1 & & 1 & & & 19 & 428 & 429 & 19 & $80 \%$ \\
\hline BB1-20a & & 1 & & 1 & & 1 & 20 & 430 & 431 & 20 & $90 \%$ \\
\hline $\mathrm{b}$ & & 1 & & 1 & & 1 & 20 & 430 & 431 & 20 & $90 \%$ \\
\hline $\mathrm{c}$ & & 1 & & 1 & & 1 & 20 & 430 & 431 & 20 & $90 \%$ \\
\hline
\end{tabular}




\begin{tabular}{|c|c|c|c|c|c|c|c|c|c|c|c|}
\hline Bead & $\begin{array}{c}\mathrm{T3B} \\
\mathrm{C} 5 \mathrm{Cl3}\end{array}$ & \begin{tabular}{|l}
$\mathrm{T} 4 \mathrm{~B}$ \\
$\mathrm{C} 6 \mathrm{C} 13$
\end{tabular} & $\begin{array}{l}\text { T2A } \\
\text { C4C15 }\end{array}$ & $\begin{array}{l}\text { T3A } \\
\text { C5C15 }\end{array}$ & \begin{tabular}{|l} 
T4A \\
C6C15
\end{tabular} & $\begin{array}{c}\text { T5A } \\
\text { C7C15 }\end{array}$ & $\begin{array}{c}\mathrm{BB} \\
\text { (expect) }\end{array}$ & $\underset{\text { (calcd) }}{\mathrm{MW}}$ & $\begin{array}{c}\text { MW } \\
\text { (LC-MS) }\end{array}$ & $\begin{array}{c}\text { confirmed } \\
\mathrm{BB}\end{array}$ & $\begin{array}{l}\text { purity of } \\
\text { product }\end{array}$ \\
\hline BB 1-21a & & 1 & & 1 & 1 & & 21 & 480 & 481 & 21 & $90 \%$ \\
\hline b & & 1 & & 1 & 1 & & 21 & 480 & 481 & 21 & $90 \%$ \\
\hline c & & 1 & & 1 & 1 & & 21 & 480 & 481 & 21 & $90 \%$ \\
\hline BB1-22a & & 1 & & 1 & 1 & 1 & 22 & 492 & 493 & 22 & $90 \% 8$ \\
\hline b & & 1 & & 1 & 1 & 1 & 22 & 492 & 493 & 22 & $90 \%$ \\
\hline $\mathrm{c}$ & & 1 & & 1 & 1 & 1 & 22 & 492 & 493 & 22 & $90 \%$ \\
\hline BB1-23a & & 1 & 1 & & & & 23 & 478 & 479 & 23 & $90 \% 8$ \\
\hline $\mathrm{b}$ & & 1 & 1 & & & & 23 & 478 & 479 & 23 & $90 \%$ \\
\hline c & & 1 & 1 & & & & 23 & 478 & 479 & 23 & $90 \%$ \\
\hline BB1-24a & & 1 & 1 & & & 1 & 24 & 463 & 464 & 24 & $90 \%$ \\
\hline $\mathrm{b}$ & & 1 & 1 & & & 1 & 24 & 463 & 464 & 24 & $90 \%$ \\
\hline c & & 1 & 1 & & & 1 & 24 & 463 & 464 & 24 & $90 \%$ \\
\hline BB1-25a & & 1 & 1 & & 1 & & 25 & 586 & 587 & 25 & $80 \%$ \\
\hline $\mathrm{b}$ & & 1 & 1 & & 1 & & 25 & 586 & 587 & 25 & $80 \%$ \\
\hline c & & 1 & 1 & & 1 & & 25 & 586 & 587 & 25 & $80 \%$ \\
\hline BB1-26a & & 1 & 1 & & 1 & 1 & 26 & 480 & 481 & 26 & $70 \%$ \\
\hline $\mathrm{b}$ & & 1 & 1 & & 1 & 1 & 26 & 480 & 481 & 26 & $70 \%$ \\
\hline $\mathrm{c}$ & & 1 & 1 & & 1 & 1 & 26 & 480 & 481 & 26 & $70 \% 8$ \\
\hline BB1-27a & & 1 & 1 & 1 & & & 27 & 387 & 388 & 27 & $90 \%$ \\
\hline $\mathrm{b}$ & & 1 & 1 & 1 & & & 27 & 387 & 388 & 27 & $90 \%$ \\
\hline c & & 1 & 1 & 1 & & & 27 & 387 & 388 & 27 & $90 \%$ \\
\hline BB1-28a & & 1 & 1 & 1 & & 1 & 28 & 435 & 436 & 28 & $90 \%$ \\
\hline $\mathrm{b}$ & & 1 & 1 & 1 & & 1 & 28 & 435 & 436 & 28 & $90 \%$ \\
\hline $\mathrm{c}$ & & 1 & 1 & 1 & & 1 & 28 & 435 & 436 & 28 & $90 \%$ \\
\hline BB1-29a & & 1 & 1 & 1 & 1 & & 29 & 439 & 440 & 29 & $90 \%$ \\
\hline $\mathrm{b}$ & & 1 & 1 & 1 & 1 & & 29 & 439 & 440 & 29 & $90 \%$ \\
\hline c & & 1 & 1 & 1 & 1 & & 29 & 439 & 440 & 29 & $90 \%$ \\
\hline BB 1-30a & & 1 & 1 & 1 & 1 & 1 & 30 & 413 & 414 & 30 & $90 \%$ \\
\hline $\mathrm{b}$ & & 1 & 1 & 1 & 1 & 1 & 30 & 413 & 414 & 30 & $90 \%$ \\
\hline c & & 1 & 1 & 1 & 1 & 1 & 30 & 413 & 414 & 30 & $90 \%$ \\
\hline BB1-31a & 1 & & & & & & 31 & 419 & 420 & 31 & 6098 \\
\hline $\mathrm{b}$ & 1 & & & & & & 31 & 419 & 420 & 31 & $60 \%$ \\
\hline c & 1 & & & & & & 31 & 419 & 420 & 31 & 6098 \\
\hline BB1-32a & 1 & & & & & 1 & 32 & 359 & 360 & 32 & $90 \%$ \\
\hline $\mathrm{b}$ & 1 & & & & & 1 & 32 & 359 & 360 & 32 & $90 \%$ \\
\hline c & 1 & & & & & 1 & 32 & 359 & 360 & 32 & $90 \%$ \\
\hline BB1-33a & 1 & & & & 1 & & 33 & 389 & 390 & 33 & $90 \%$ \\
\hline $\mathrm{b}$ & 1 & & & & 1 & & 33 & 389 & 390 & 33 & $90 \%$ \\
\hline $\mathrm{c}$ & 1 & & & & 1 & & 33 & 389 & 390 & 33 & $90 \%$ \\
\hline BB1-34a & 1 & & & & 1 & 1 & 34 & 465 & 466 & 34 & $90 \%$ \\
\hline $\mathrm{b}$ & 1 & & & & 1 & 1 & 34 & 465 & 466 & 34 & $90 \%$ \\
\hline c & 1 & & & & 1 & 1 & 34 & 465 & 466 & 34 & $90 \%$ \\
\hline BB1-35a & 1 & & & 1 & & & 35 & 453 & 454 & 35 & $90 \%$ \\
\hline $\mathrm{b}$ & 1 & & & 1 & & & 35 & 453 & 454 & 35 & $90 \%$ \\
\hline c & 1 & & & 1 & & & 35 & 453 & 454 & 35 & $90 \%$ \\
\hline BB1-36a & 1 & & & 1 & & 1 & 36 & 401 & 402 & 36 & $90 \% 8$ \\
\hline $\mathrm{b}$ & 1 & & & 1 & & 1 & 36 & 401 & 402 & 36 & $90 \%$ \\
\hline c & 1 & & & 1 & & 1 & 36 & 401 & 402 & 36 & $90 \%$ \\
\hline \begin{tabular}{|l} 
BB1-37a \\
\end{tabular} & 1 & & & 1 & 1 & & 37 & 407 & 408 & 37 & $90 \%$ \\
\hline $\mathrm{b}$ & 1 & & & 1 & 1 & & 37 & 407 & 408 & 37 & $90 \%$ \\
\hline c & 1 & & & 1 & 1 & & 37 & 407 & 408 & 37 & $90 \%$ \\
\hline \begin{tabular}{|l|} 
BB 1-38a \\
\end{tabular} & 1 & & & 1 & 1 & 1 & 38 & 467 & 468 & 38 & $90 \%$ \\
\hline $\mathrm{b}$ & 1 & & & 1 & 1 & 1 & 38 & 467 & 468 & 38 & $90 \%$ \\
\hline c & 1 & & & 1 & 1 & 1 & 38 & 467 & 468 & 38 & $90 \%$ \\
\hline BB1-39a & 1 & & 1 & & & & 39 & 491 & 492 & 39 & $90 \%$ \\
\hline $\mathrm{b}$ & 1 & & 1 & & & & 39 & 491 & 492 & 39 & $90 \%$ \\
\hline c & 1 & & 1 & & & & 39 & 491 & 492 & 39 & $90 \%$ \\
\hline BB1-40a & 1 & & 1 & & & 1 & 40 & 457 & 458 & 40 & $90 \%$ \\
\hline $\mathrm{b}$ & 1 & & 1 & & & 1 & 40 & 457 & 458 & 40 & $90 \%$ \\
\hline c & 1 & & 1 & & & 1 & 40 & 457 & 458 & 40 & $90 \%$ \\
\hline \begin{tabular}{|l} 
BB 1-41a \\
\end{tabular} & 1 & & 1 & & 1 & & 41 & 427 & 428 & 41 & $90 \%$ \\
\hline $\mathrm{b}$ & 1 & & 1 & & 1 & & 41 & 427 & 428 & 41 & $90 \%$ \\
\hline $\mathrm{c}$ & 1 & & 1 & & 1 & & 41 & 427 & 428 & 41 & $90 \%$ \\
\hline
\end{tabular}




\section{(7-3) Amine modification (Second step, synthesis of 5)}

31 Portions of beads reacted with thiols and secondary amines at the previous step (BB1-1 to 31) were combined, and subjected to the second tagging step. 10 Portions of beads reacted with primary amines at the previous step were pooled and split into 14 portions. Each pool of beads was treated with an electrophilic building block in the second step, and tagged. The detail reaction condition and the binary encoding strategy were shown in Table SI-3.

\section{Table SI-3}

\begin{tabular}{|c|c|c|c|c|c|c|c|c|c|c|c|}
\hline BB-No. & structure & MW & density & vendor & $\begin{array}{l}\text { No. of } \\
\text { beads }\end{array}$ & $\begin{array}{l}\text { amount for } \\
\text { reaction }\end{array}$ & other conditions & $\begin{array}{c}\text { T6A } \\
\text { C8C15 }\end{array}$ & $\begin{array}{c}\text { T7A } \\
\text { C9C15 }\end{array}$ & $\begin{array}{c}\text { T8A } \\
\text { C10C15 }\end{array}$ & $\begin{array}{r}\text { T9A } \\
\text { C11Cls }\end{array}$ \\
\hline BB2-1 & no reagent & & & & 4650 & & & & & & 1 \\
\hline BB2-2 & & 214.65 & & Ald. & 1500 & $644 \mathrm{mg}$ & $\begin{array}{c}\mathrm{CH} 2 \mathrm{Cl} 215 \mathrm{~mL} \\
\text { DIPEA } 0.784 \mathrm{~mL} \\
20 \mathrm{hr}\end{array}$ & & & 1 & \\
\hline BB2-3 & & 216.66 & & Ald. & 1500 & $650 \mathrm{mg}$ & $\begin{array}{c}\mathrm{CH} 2 \mathrm{Cl} 215 \mathrm{~mL} \\
\text { DIPEA } 0.784 \mathrm{~mL} \\
20 \mathrm{hr}\end{array}$ & & & 1 & 1 \\
\hline BB2-4 & & 149.15 & 1.151 & Ald. & 1500 & $\begin{array}{c}447 \mathrm{mg} \\
0.389 \mathrm{~mL}\end{array}$ & $\begin{array}{c}\mathrm{CH} 2 \mathrm{Cl} 215 \mathrm{~mL} \\
20 \mathrm{hr}\end{array}$ & & 1 & & \\
\hline BB2-5 & & 85.11 & 0.866 & Ald. & 1500 & $\begin{array}{r}255 \mathrm{mg} \\
0.294 \mathrm{~mL}\end{array}$ & $\begin{array}{c}\mathrm{CH} 2 \mathrm{Cl} 215 \mathrm{~mL} \\
20 \mathrm{hr}\end{array}$ & & 1 & & 1 \\
\hline BB2-6 & & 119.12 & 1.096 & Ald. & 1500 & $\begin{array}{r}357 \mathrm{mg} \\
0.326 \mathrm{~mL}\end{array}$ & $\begin{array}{c}\mathrm{CH} 2 \mathrm{Cl} 215 \mathrm{~mL} \\
20 \mathrm{hr}\end{array}$ & & 1 & 1 & \\
\hline BB2-7 & & 147.17 & 1.045 & Ald. & 1500 & $\begin{array}{r}442 \mathrm{mg} \\
0.423 \mathrm{~mL}\end{array}$ & $\begin{array}{c}\mathrm{CH} 2 \mathrm{Cl} 215 \mathrm{~mL} \\
20 \mathrm{hr}\end{array}$ & & 1 & 1 & 1 \\
\hline BB2-8 & & 114.55 & 1.48 & Ald. & 1500 & $\begin{array}{c}344 \mathrm{mg} \\
0.232 \mathrm{~mL}\end{array}$ & $\begin{array}{c}\text { CH2Cl2 } 15 \mathrm{~mL} \\
\text { DMAP } 550 \mathrm{mg} \\
20 \mathrm{hr}\end{array}$ & 1 & & & \\
\hline BB2-9 & & 182.65 & & Ald. & 1500 & $548 \mathrm{mg}$ & $\begin{array}{c}\text { CH2Cl2 } 15 \mathrm{~mL} \\
\text { DMAP } 550 \mathrm{mg} \\
20 \mathrm{hr}\end{array}$ & 1 & & & 1 \\
\hline BB2-10 & & 74.08 & 1.117 & Ald. & 1500 & $\begin{array}{c}222 \mathrm{mg} \\
0.199 \mathrm{~mL}\end{array}$ & $\begin{array}{c}\text { IPA } 3.75 \mathrm{~mL} \\
20 \mathrm{hr}\end{array}$ & 1 & & 1 & \\
\hline BB2-11 & & 164.2 & 1.077 & Ald. & 1500 & $\begin{array}{r}493 \mathrm{mg} \\
0.458 \mathrm{~mL}\end{array}$ & $\begin{array}{c}\text { IPA } 3.75 \mathrm{~mL} \\
20 \mathrm{hr}\end{array}$ & 1 & & 1 & 1 \\
\hline BB2-12 & & 108.52 & 1.135 & Ald. & 1500 & $\begin{array}{r}326 \mathrm{mg} \\
0.287 \mathrm{~mL}\end{array}$ & $\begin{array}{c}\mathrm{CH} 2 \mathrm{Cl} 215 \mathrm{~mL} \\
\text { DIPEA } 0.784 \mathrm{~mL} \\
20 \mathrm{hr}\end{array}$ & 1 & 1 & & \\
\hline BB2-13 & & 206.62 & 1.273 & TCI & 1500 & $\begin{array}{l}620 \mathrm{mg} \\
0.487 \mathrm{~mL}\end{array}$ & $\begin{array}{c}\mathrm{CH} 2 \mathrm{Cl} 15 \mathrm{~mL} \\
\text { DIPEA } 0.784 \mathrm{~mL} \\
20 \mathrm{hr}\end{array}$ & 1 & 1 & & 1 \\
\hline BB2-14 & & 218.73 & 1.02 & Ald. & 1500 & $\begin{array}{r}656 \mathrm{mg} \\
0.643 \mathrm{~mL}\end{array}$ & $\begin{array}{c}\mathrm{CH} 2 \mathrm{Cl} 215 \mathrm{~mL} \\
\text { DIPEA } 0.784 \mathrm{~mL} \\
20 \mathrm{hr}\end{array}$ & 1 & 1 & 1 & \\
\hline BB2-15 & carbonyl diimidazol & 162.15 & & Ald. & 1500 & $486 \mathrm{mg}$ & $\begin{array}{l}\text { THF } 15 \mathrm{~mL} \\
\text { NaH } 60 \mathrm{mg} \\
20 \mathrm{hr}\end{array}$ & 1 & 1 & 1 & 1 \\
\hline
\end{tabular}




\section{(7-4) Quality check after the second step}

Three beads were selected from each portion ( $3 \times 15=45$ beads). The compounds on the beads were cleaved individually. While the compounds dissolved in $\mathrm{MeOH}(100 \mathrm{ml})$ were analyzed by LC-MS, Tags on the beads were cleaved and analyzed by GC. The results are shown in Table SI-4.

\section{Table SI-4}

\begin{tabular}{|c|c|c|c|c|c|c|c|c|c|c|c|c|c|c|c|c|c|}
\hline Bead & \begin{tabular}{|c|} 
T3B \\
C5C13
\end{tabular} & $\begin{array}{c}\mathrm{T} 4 \mathrm{~B} \\
\mathrm{C} 6 \mathrm{Cl3}\end{array}$ & $\begin{array}{c}\text { T2A } \\
\text { C4C15 }\end{array}$ & $\begin{array}{c}\text { T3A } \\
\text { C5C15 }\end{array}$ & $\begin{array}{l}\text { T4A } \\
\text { C6C15 }\end{array}$ & $\begin{array}{l}\text { T5A } \\
\text { C7C15 }\end{array}$ & \begin{tabular}{|c|} 
BB1 \\
(expect)
\end{tabular} & $\begin{array}{l}\text { T6A } \\
\text { C8C15 }\end{array}$ & \begin{tabular}{|l|}
$77 \mathrm{~A}$ \\
$\mathrm{C9C15}$ \\
\end{tabular} & \begin{tabular}{|c|} 
T8A \\
C10C15
\end{tabular} & \begin{tabular}{|c|} 
T9A \\
C11C15 \\
\end{tabular} & \begin{tabular}{|c|}
$\mathrm{BB} 2$ \\
(expect)
\end{tabular} & $\begin{array}{c}\text { MW } \\
\text { (calcd) }\end{array}$ & $\begin{array}{c}\text { MW } \\
\text { (LCMS+) }\end{array}$ & \begin{tabular}{|c|}
$\begin{array}{c}\text { confirmed } \\
\text { BB1 }\end{array}$ \\
\end{tabular} & \begin{tabular}{|c|}
$\begin{array}{c}\text { confirmed } \\
\text { BB2 }\end{array}$ \\
\end{tabular} & $\begin{array}{c}\text { purity of } \\
\text { product }\end{array}$ \\
\hline BB2-1a & & 1 & 1 & 1 & 1 & & 29 & & & & 1 & 1 & 439 & 440 & 29 & 1 & $90 \%$ \\
\hline b & & 1 & 1 & 1 & 1 & 1 & 30 & & & & 1 & 1 & 413 & 440 & 29 & 1 & $80 \%$ \\
\hline c & & 1 & & & & 1 & 16 & & & & 1 & 1 & 511 & 512 & 16 & 1 & $80 \%$ \\
\hline BB2-2a & 1 & & 1 & & 1 & & 41 & & & 1 & & 2 & 605 & 606 & 41 & 2 & $90 \%$ \\
\hline $\mathrm{b}$ & 1 & & & 1 & & & 35 & & & 1 & & 2 & 631 & 632 & 35 & 2 & $90 \%$ \\
\hline $\mathrm{c}$ & 1 & & & 1 & 1 & & 37 & & & 1 & & 2 & 585 & 586 & 37 & 2 & $90 \%$ \\
\hline BB2-3a & 1 & & & 1 & & & 35 & & & 1 & 1 & 3 & 633 & 634 & 35 & 3 & $80 \%$ \\
\hline b & 1 & & 1 & & 1 & & 41 & & & 1 & 1 & 3 & 607 & 608 & 41 & 3 & $80 \%$ \\
\hline c & 1 & & & & & 1 & 32 & & & 1 & 1 & 3 & 539 & 540 & 32 & 3 & $80 \%$ \\
\hline BB2-4a & 1 & & & & 1 & 1 & 34 & & 1 & & & 4 & 614 & 615 & 34 & 4 & $80 \%$ \\
\hline b & 1 & & & 1 & & 1 & 40 & & 1 & & & 4 & 606 & 607 & 40 & 4 & $70 \%$ \\
\hline c & 1 & & & 1 & 1 & 1 & 41 & & 1 & & & 4 & 576 & 577 & 41 & 4 & $80 \%$ \\
\hline BB2-5a & 1 & & & 1 & & & 35 & & 1 & & 1 & 5 & 538 & 539 & 35 & 5 & $70 \%$ \\
\hline b & 1 & & 1 & & 1 & & 41 & & 1 & & 1 & 5 & 512 & 513 & 41 & 5 & $80 \%$ \\
\hline $\mathrm{c}$ & 1 & & 1 & & 1 & & 41 & & 1 & & 1 & 5 & 512 & 513 & 41 & 5 & $80 \%$ \\
\hline BB2-6a & 1 & & & 1 & 1 & & 37 & & 1 & 1 & & 6 & 526 & 527 & 37 & 6 & $90 \%$ \\
\hline b & 1 & & & & 1 & & 33 & & 1 & 1 & & 6 & 508 & 509 & 33 & 6 & $80 \%$ \\
\hline c & 1 & & & 1 & & 1 & 36 & & 1 & 1 & & 6 & 520 & 521 & 36 & 6 & $70 \%$ \\
\hline BB2-7a & 1 & & & & 1 & & 33 & & 1 & 1 & 1 & 7 & 536 & 537 & 33 & 7 & $70 \%$ \\
\hline b & 1 & & & 1 & & 1 & 36 & & 1 & 1 & 1 & 7 & 548 & 549 & 36 & 7 & $50 \%$ \\
\hline c & 1 & & & 1 & & & 35 & & 1 & 1 & 1 & 7 & 600 & 601 & 35 & 7 & $60 \%$ \\
\hline BB2-8a & 1 & & & & 1 & 1 & 34 & 1 & & & & 8 & 543 & 478 & 34 & 8 & $40 \%$ \\
\hline b & 1 & & & & & 1 & 32 & 1 & & & & 8 & 437 & 420 & 32 & 8 & $50 \%$ \\
\hline c & 1 & & & 1 & & & 35 & 1 & & & & 8 & 531 & 514 & 35 & 8 & $60 \%$ \\
\hline BB2-9a & 1 & & 1 & & & & 39 & 1 & & & 1 & 9 & 637 & 620 & 39 & 9 & $80 \%$ \\
\hline b & 1 & & 1 & & & & 39 & 1 & & & 1 & 9 & 637 & 620 & 39 & 9 & 8098 \\
\hline $\mathrm{c}$ & 1 & & & 1 & & & 35 & 1 & & & 1 & 9 & 599 & 580 & 35 & 9 & $70 \%$ \\
\hline BB2-10a & 1 & & & & 1 & & 33 & 1 & & 1 & & 10 & 463 & 464 & 33 & 10 & $80 \%$ \\
\hline b & 1 & & & 1 & & 1 & 36 & 1 & & 1 & & 10 & 475 & 476 & 36 & 10 & $70 \%$ \\
\hline c & 1 & & 1 & & & 1 & 40 & 1 & & 1 & & 10 & 531 & 532 & 40 & 10 & $80 \%$ \\
\hline BB2-11a & & & & & & & 0 & & & & & 0 & 0 & 572 & 37 & 11 & $90 \%$ \\
\hline b & 1 & & & & & 1 & 32 & 1 & & 1 & 1 & 11 & 523 & 524 & 32 & 11 & $90 \%$ \\
\hline $\mathrm{c}$ & 1 & & & 1 & 1 & & 37 & 1 & & 1 & 1 & 11 & 571 & 572 & 37 & 11 & $909 \%$ \\
\hline BB2-12a & 1 & & 1 & & 1 & & 41 & 1 & 1 & & & 12 & 499 & 500 & 41 & 12 & 8098 \\
\hline b & 1 & & 1 & & 1 & & 41 & 1 & 1 & & & 12 & 499 & 500 & 41 & 12 & $80 \%$ \\
\hline c & 1 & & & 1 & 1 & 1 & 38 & 1 & 1 & & & 12 & 539 & 540 & 38 & 12 & $80 \%$ \\
\hline BB2-13a & 1 & & & & & 1 & 32 & 1 & 1 & & 1 & 13 & 529 & 530 & 32 & 13 & $70 \%$ \\
\hline b & 1 & & & & 1 & & 33 & 1 & 1 & & 1 & 13 & 559 & 560 & 33 & 13 & $70 \%$ \\
\hline c & 1 & & 1 & & 1 & & 41 & 1 & 1 & & 1 & 13 & 597 & 598 & 41 & 13 & $80 \%$ \\
\hline BB2-14a & 1 & & & & & 1 & 32 & 1 & 1 & 1 & & 14 & 541 & 542 & 32 & 14 & $60 \%$ \\
\hline b & 1 & & 1 & & & 1 & 40 & 1 & 1 & 1 & & 14 & 639 & 640 & 40 & 14 & $90 \%$ \\
\hline c & 1 & & 1 & & 1 & & 41 & 1 & 1 & 1 & & 14 & 609 & 610 & 41 & 14 & $90 \%$ \\
\hline BB2-15a & 1 & & & 1 & & & 35 & 1 & 1 & 1 & 1 & 15 & 479 & 480 & 35 & 15 & $90 \%$ \\
\hline b & 1 & & 1 & & 1 & & 41 & 1 & 1 & 1 & 1 & 15 & 453 & 454 & 41 & 15 & 9098 \\
\hline c & 1 & & & & & 1 & 32 & 1 & 1 & 1 & 1 & 15 & 385 & 386 & 32 & 15 & $90 \%$ \\
\hline
\end{tabular}


(7-5) Diels-Alder reaction (Third step, synthesis of 6)

All beads derived from the second step were combined. $4 \%$ (weight) of the beads were removed from the pool of beads and stored as the first part of library. The remaining beads were divided into 12 portions. Each pool of beads was subjected to Diels-Alder reaction with the third building blocks (ynones), and tagged. The detail reaction condition and the binary encoding strategy were shown in Table SI-5.

Table SI-5

\begin{tabular}{|c|c|c|c|c|c|c|c|c|c|}
\hline BB-No. & structure & MW & $\begin{array}{c}\text { No. of } \\
\text { beads }\end{array}$ & $\begin{array}{l}\text { amount for } \\
\text { reaction }\end{array}$ & other conditions & $\begin{array}{c}\text { T5B } \\
\text { C7Cl3 }\end{array}$ & $\begin{array}{c}\text { T10A } \\
\text { C12C15 }\end{array}$ & $\begin{array}{c}\text { T13A } \\
\text { C15C15 }\end{array}$ & $\begin{array}{c}\text { T14A } \\
\text { C16C15 }\end{array}$ \\
\hline BB3-1 & & 130.14 & 2052 & $268 \mathrm{mg}$ & $\begin{array}{c}\mathrm{CH} 2 \mathrm{Cl} 240 \mathrm{~mL} \\
\mathrm{E} 2 \mathrm{AlCl} 8.26 \mathrm{ml} \\
4 \mathrm{hr}\end{array}$ & & & & 1 \\
\hline BB3-2 & & 166.12 & 2052 & $342 \mathrm{mg}$ & $\begin{array}{c}\mathrm{CH} 2 \mathrm{Cl} 240 \mathrm{~mL} \\
\mathrm{E} 2 \mathrm{AlCl} 8.26 \mathrm{ml} \\
4 \mathrm{hr}\end{array}$ & & & 1 & \\
\hline BB3-3 & & 150.2 & 2052 & $309 \mathrm{mg}$ & $\begin{array}{c}\mathrm{CH} 2 \mathrm{Cl} 240 \mathrm{~mL} \\
\mathrm{E} 2 \mathrm{AlCl} 8.26 \mathrm{ml} \\
4 \mathrm{hr}\end{array}$ & & & 1 & 1 \\
\hline BB3-4 & & 186.23 & 2052 & $384 \mathrm{mg}$ & $\begin{array}{c}\mathrm{CH} 2 \mathrm{Cl} 240 \mathrm{~mL} \\
\mathrm{E} 2 \mathrm{AlCl} 8.26 \mathrm{ml} \\
4 \mathrm{hr}\end{array}$ & 1 & 1 & 1 & \\
\hline BB3-5 & & 198.14 & 2052 & $1.63 \mathrm{~g}$ & $\begin{array}{c}\mathrm{CH} 2 \mathrm{Cl2} 30 \mathrm{~mL} \\
\mathrm{E} 2 \mathrm{AlCl} 8.26 \mathrm{ml} \\
12 \mathrm{hr}\end{array}$ & & 1 & & 1 \\
\hline BB3-6 & & 136.19 & 2052 & $281 \mathrm{mg}$ & $\begin{array}{c}\mathrm{CH} 2 \mathrm{Cl} 240 \mathrm{~mL} \\
\mathrm{E} 2 \mathrm{AlCl} 8.26 \mathrm{ml} \\
4 \mathrm{hr}\end{array}$ & & 1 & 1 & \\
\hline BB3-7 & & 120.11 & 2052 & $247 \mathrm{mg}$ & $\begin{array}{c}\mathrm{CH} 2 \mathrm{Cl} 240 \mathrm{~mL} \\
\mathrm{E} 2 \mathrm{AlCl} 8.26 \mathrm{ml} \\
4 \mathrm{hr}\end{array}$ & & 1 & 1 & 1 \\
\hline BB3-8 & & 160.17 & 2052 & $330 \mathrm{mg}$ & $\begin{array}{c}\mathrm{CH} 2 \mathrm{Cl} 240 \mathrm{~mL} \\
\mathrm{E} 22 \mathrm{AlCl} 8.26 \mathrm{ml} \\
4 \mathrm{hr}\end{array}$ & 1 & & & \\
\hline BB3-9 & & 206.24 & 2052 & $428 \mathrm{mg}$ & $\begin{array}{c}\mathrm{CH} 2 \mathrm{Cl} 240 \mathrm{~mL} \\
\mathrm{E} 12 \mathrm{AlCl} 8.26 \mathrm{ml} \\
4 \mathrm{hr}\end{array}$ & 1 & & & 1 \\
\hline BB3-10 & & 298.64 & 2052 & $2.46 \mathrm{~g}$ & $\begin{array}{c}\mathrm{CH} 2 \mathrm{Cl} 230 \mathrm{~mL} \\
\mathrm{E} 2 \mathrm{AlCl} 8.26 \mathrm{ml} \\
12 \mathrm{hr}\end{array}$ & 1 & & 1 & \\
\hline BB3-11 & & 196.61 & 2052 & $1.62 \mathrm{~g}$ & $\begin{array}{c}\mathrm{CH} 2 \mathrm{Cl} 230 \mathrm{~mL} \\
\mathrm{E}+2 \mathrm{AlCl} 8.26 \mathrm{ml} \\
12 \mathrm{hr}\end{array}$ & 1 & & 1 & 1 \\
\hline BB3-12 & & 206.24 & 2052 & $428 \mathrm{mg}$ & $\begin{array}{c}\mathrm{CH} 2 \mathrm{Cl} 240 \mathrm{~mL} \\
\mathrm{E} 2 \mathrm{AlCl} 8.26 \mathrm{ml} \\
4 \mathrm{hr}\end{array}$ & 1 & 1 & 1 & 1 \\
\hline
\end{tabular}




\section{(7-6) Quality check after the third step}

Three beads were selected from each portion $(3 \times 12=36$ beads). The compounds on the beads were cleaved individually. While the compounds dissolved in $\mathrm{MeOH}(100 \mathrm{ml})$ were analyzed by LC-MS, Tags on the beads were cleaved and analyzed by GC. The results are shown in Table SI-6.

Table SI-6

\begin{tabular}{|c|c|c|c|c|c|c|c|c|c|c|c|c|c|c|c|c|c|c|c|c|c|c|c|c|c|c|c|c|c|c|c|}
\hline$\therefore$ & 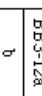 & & . & 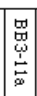 & & $\sigma$ & 思 &. & 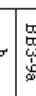 & & $\sigma$ & 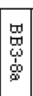 & & & 象 & & 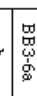 & & $\sigma$ & 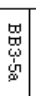 & & & & & & & & & & & 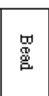 \\
\hline & - & & - & - & - & & - & - & - & - & & - & - & - & - & - & - & & - & - & & & - & - & & & & - & - & & 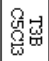 \\
\hline & & 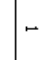 & & & & & & & - & & & & & t & & & & $r$ & & & & & & & & & - & & & & 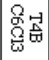 \\
\hline - & $\rightarrow r$ & & & & & - & & . & - & & - & - & & $r$ & & & - & & & - & - & - & & & & & $r$ & & - & & 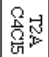 \\
\hline & & - & - & & & & - & & - & - & & & & & & & & & - & & - & 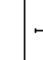 & & & & - & 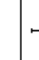 & & & & 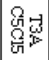 \\
\hline- & $-\rightarrow$ & - & & & - & & & & & - & - & & - & & - & - & - & & & & - & 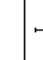 & & & & - & r & & & & 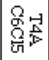 \\
\hline- & & - & - & & & - & & - & 1 & & & - & - & & - & & & & - & & & & & - & & & -1 & - & & & $\begin{array}{l}300 \\
30 \% \\
5\end{array}$ \\
\hline s & $\Rightarrow 0$ & $\bar{\omega} \mid \mathcal{\omega}$ & $\mathscr{\alpha}$ & 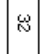 & $\Rightarrow$ & $\infty$ & 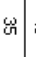 & $\Delta:$ & $\approx$ & $\omega$ & $\circ$ & $A$ & ๙ & & $\stackrel{L}{L}=$ & $\Rightarrow \Rightarrow$ & $\Rightarrow$ & $\overrightarrow{0}$ & $\%$ & 思 & $\vec{\omega}$ & & $\Delta$ & $\Psi$ & a & ฯ & $\underset{\sim}{\Omega}$ & & $\omega$ & & 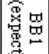 \\
\hline & - & & - & & - & & & & - & - & & - & & & - & - & - & & & - & & 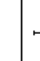 & - & & & & r & - & - & & 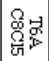 \\
\hline & - & & - & - & - & & - &.- & - & & & & - & & & - & & & - & & & & & - & & & & - & & & 恋: \\
\hline & - & & $r$ & & - & & - & - & - & $r$ & & & - & , & - & $r$ & & & - & - & & & & - & & & & - & - & & 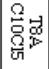 \\
\hline r & - & - & - & - & & - & - & & 1 & & - & - & - & & & -1 & & - & & & - & & - & - & & - & - & - & - & & $\begin{array}{l}2 \\
\overline{3} \\
3 \\
3\end{array}$ \\
\hline- & $\vec{ज}$ & -- & $\vec{v}$ & a & $\vec{a}$ & - & $\sim$ & $\sigma=$ & - & a & - & $\circ$ & $\sim$ & - & ज 0 & $\vec{c}$ & $\infty$ & - & . & $a$ & - & $-\infty$ & $\Delta$ & $\sim$ & - & - & $=$ & $=0$ & $=$ & & 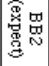 \\
\hline- & - & - & - & - & - & - & - & - & -1. & - & - & - & & & & & & & & & 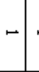 & & - & & & & & & & & 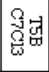 \\
\hline$r$ & - & & & & & & & & & & & & - & - & & $\rightarrow$ & - & r & - & - & 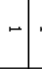 & & & & & & & & & & 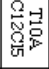 \\
\hline - & -7 & - & ـ & - & - & - & - & & & & & & - & - & - & - & - & & & & - & - & -- & - & - & - & - & & & & 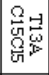 \\
\hline- & - & - & - & & & & & -1 & $-r$ & & & & - & - & & & & - & . & - & & & & - & - & & & & - & & 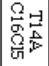 \\
\hline$\vec{N}$ & $\vec{\sim}$ & $\vec{N}=$ & $=$ & $=$ & $a$ & 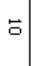 & 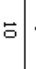 &. &. & $\infty$ & $\infty$ & $\infty$ & $\sim$ & $\sim$ & $\sim 0$ & 0.0 & 0 & or & or & ( & $\triangle$ & $\Delta$ & $\Delta \omega$ & $\omega$ & $\omega$ & $N$ & $N N$ & & - & - & 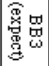 \\
\hline 尊 & 品 & 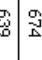 & 胥 & 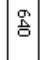 & 恕 & $\stackrel{\Omega}{\mathcal{G}}$ & $\stackrel{8}{\infty}$ & 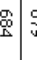 & 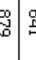 & 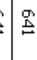 & I & $\vec{\omega}$ & 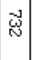 & 宫 & $g$ & $\begin{array}{l}g \\
\end{array}$ & 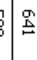 & 跑 & $\overrightarrow{\mathrm{D}}$ & హ) & $\begin{array}{l}9 \\
0 \\
0\end{array}$ & 䍐 & $\underset{c}{g}$ & 鬲 & 応 & 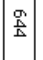 & & जे & 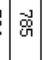 & 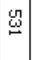 & 酸廷 \\
\hline 登 & g & 8 & $\mathbb{R}_{N}$ & 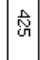 & 总 & 胥 & : & \begin{tabular}{c}
$g^{\prime}$ \\
\hdashline
\end{tabular} & $\begin{array}{c}0 \\
\end{array}$ & $\frac{D}{N}$ & 赑 & 胥 & 心 & 宵 & 茎 & g. & $\begin{array}{l}n \\
\end{array}$ & g & 常 & 恿 & 莡 & I & 염 & 学 & 总 & 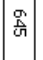 & 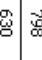 & & $: \approx$ & 心 & $\begin{array}{l}33 \\
35 \\
4\end{array}$ \\
\hline s & $\Rightarrow 0$ & $\vec{\omega} \omega$ & $\omega$ & $\approx$ & $\omega$ & $\infty$ & 兒 & 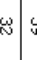 & 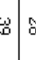 & $\omega$ & 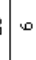 & 하 & 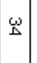 & in & $\begin{array}{l}\omega \\
\perp\end{array}$ & $\omega \nRightarrow$ & $\Rightarrow$ & $\omega$ & $\%$ & $\omega$ & $\vec{\omega}$ & & & & a & $\backsim$ & & & 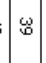 &. & 罚总 \\
\hline- & & - & $\overrightarrow{\mathrm{c}}$ & & $\overrightarrow{\mathrm{s}}$ & - & $\sim$ & o. & 5. & $a$ & - &. & $\sim$ & $\rightarrow$ & ज 0 & al $\vec{c}$ & $\infty$ & - & 0 & $\vec{a}$ & - & $-\infty$ & - 1 & $\sim$ & & - & $-=$ & & $=$ & - & 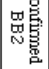 \\
\hline$\vec{N}$ & $=$ & $\vec{N}=$ & $=$ & $E$ & 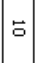 & $\vec{\sigma}$ & 5 . & $\bullet$ & 6 & $\infty$ & $\infty$ & $\infty$ & s & $\sim 1$. & & $\sigma 0$ & $\sigma$ & or & or & & $\Delta$ & $\begin{array}{lll}A & A\end{array}$ & $\Delta$ & $\omega$ & $\omega$ & N & & & - & - & 瞄总部 \\
\hline 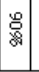 & 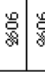 & $\begin{array}{l}8 \\
\end{array}$ & 总 & 8 & 客 & 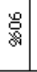 & 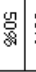 & 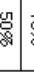 & : & 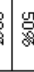 & 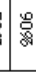 & 客 & 常 & \begin{tabular}{l|l}
$\circ$ \\
8
\end{tabular} & 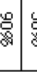 & 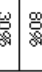 & s. & 屏 & $\begin{array}{l} \\
\\
\vdots\end{array}$ & 品 & $\stackrel{\square}{\$}$ & \begin{tabular}{l}
$Ð$ \\
\hdashline
\end{tabular} & 里 & 告 & $g$ & $\stackrel{\omega}{\%}$ & $\stackrel{8}{\circ}$ & 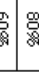 & 兑 & 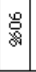 & 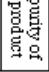 \\
\hline
\end{tabular}


(7-7) Retro-Diels-Alder reaction (Fourth step, synthesis of 7)

All beads derived from the third step were combined. The half of them was stored as the second part of library. The other half was subjected to retro-Diels-Alder reaction. The yielded pool of beads was the third part of library. 


\section{(7-8) Quality check after the fourth step}

32 Beads were selected from the third part of library. The compounds on the beads were cleaved individually. While the compounds dissolved in $\mathrm{MeOH}(100 \mathrm{ml})$ were analyzed by LC-MS, Tags on the beads were cleaved and analyzed by GC. The results are shown in Table SI-7.

Table SI-7

\begin{tabular}{|c|c|c|c|c|c|c|c|c|c|c|c|c|c|c|c|c|c|c|c|c|c|c|c|c|c|c|c|c|c|}
\hline$\underset{\Sigma}{\alpha}$ & $\omega$ & $\mathscr{\omega}$ & 8 & 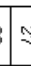 & $\approx$ & ऊ & $\tilde{\Sigma}$ & $\tilde{\omega}$ & $\mathbb{N}$ & $\cong$ & 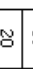 & $\overrightarrow{0}$ & $+\infty$ & के & $\stackrel{\mathrm{c}}{ }$ & B & $\grave{\omega}$ & $\vec{N}$ & $=$ & 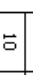 & & & & on & & & & & 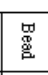 \\
\hline & & - & - & -1 & - & - & - & - & - & - & & & - & 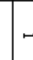 & - & - & & - & - & - & & - & & - & - & - & & & 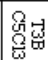 \\
\hline & & & & & & & & & & & & & r & & & & - & & & & & & & & & & & & 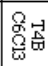 \\
\hline & - & & & & & & & - & - & - & - & - & & & & & & & & - & & & & 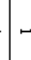 & & & - & & 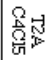 \\
\hline & & - & - & $r$ & - & - & - & & & & - & & r & & - & - & - & & & & & . & & & - & & - & & 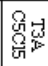 \\
\hline & & & & & $r$ & & - & - & - & & - & . & - & 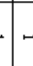 & & & - & & - & - & - & & & 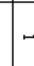 & - & & & & $\begin{array}{l}8 \\
8 \\
0,5 \\
0=1\end{array}$ \\
\hline t & & - & - & & & & - & & & - & - & - & r & & & - & & - & - & & & - & - & & - & & & & 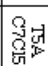 \\
\hline $\mathbb{\infty}$ & $\tilde{\omega}$ & $\%$ & $\omega$ & 3 & $\underline{\omega}$ & $\varphi_{\tilde{m}}$ & $\mathscr{\varpi}$ & $\Leftrightarrow$ & $\Leftrightarrow$ & $\mathrm{t}$ & $\vec{\Delta}$ & $\infty$ & $\omega \approx$ & $\dot{\omega}$ & 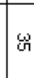 & $\%$ & $\simeq$ & 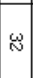 & ${ }_{\Sigma}^{\omega}$ & $\Leftrightarrow$ & $\omega$ & 心 & $\overrightarrow{0} \Delta$ & $A$ & $\mathscr{w}$ & $\omega$ & $=$ & & 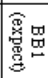 \\
\hline & & & . & & r & - & & - & - & - & & & - & r & & & & & & - & & - & r & - & - & & & & 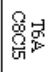 \\
\hline & & $\rightarrow$ & - & & & & - & & - & & & & - & & $r$ & - & & - & & & $\rightarrow$ & & & & & - & & & \\
\hline & & 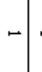 & & & r & & & - & - & - & & , & - & & & & & & - & - & & - & & - & & - & & & 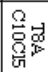 \\
\hline 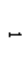 & & & & & $r$ & & - & - & & & & - & r & & & - & - & & - & - & & & - & & - & & - & & 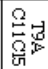 \\
\hline$\rightarrow$ & - & $\sigma$. & $\sigma 0$ & 5 & $ت$ & $\infty$ & ar & $ت$ & $\vec{s}$ & s & - & $-I$ & 5 & $\infty$ & $\triangle$ & a & - & $\triangle$ & $=$ & $\overrightarrow{\mathrm{c}}$ & & & & $=N$ & & 0 & - & & 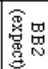 \\
\hline & - & & - & - & & & & - & & & - & & r & & - & - & - & & & & - & & & & & & & & \\
\hline & - & & & & & & & - & - & & - & & - & & & - & & & - & & & & r & & - & & & & \\
\hline & - & - & $\rightarrow r$ & & - & - & - & - & - & - & - & - & - & - & 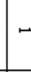 & - & 1 & - & - & - & & - & 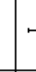 & . & & - & - & & 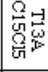 \\
\hline - & & & - & r & & - & - & & & - & & - & - & r & & & - & & - & & & & -1 & . & & & & & $\begin{array}{l}\Omega \\
\Omega \\
0 \\
0 \\
0=5\end{array}$ \\
\hline - & $\grave{N}$ & $N ;$ & $=0$ & 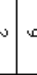 & $N$ & $\omega$ & $\omega$ & $\triangle$ & $\sigma$ & $\sim$ & $\triangle$ & $\omega$. & $=$ & 5 & t & $\Delta$ & $=$ & $N$ & $\sim$ & $\sim$ & $\infty$ & $N$ & -0 & $N$ & $\sigma$ & $N$ & $N$ & & 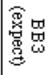 \\
\hline g & $\begin{array}{l}0 \\
0 \\
\end{array}$ & : & $\vec{\sigma}$ & $a$ & $\vec{\omega}$ & $\mathscr{g}_{\mathscr{a}}^{\circ}$ & 공 & 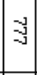 & 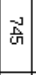 & 跣 & g. & খู & 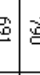 & 9 & $\because$ & 今 & \% & 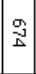 & 党 & $\frac{9}{0}$ & 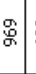 & 总 & 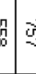 & 3 & $\mathcal{W}$ & ज. & 行 & 罢 & 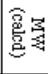 \\
\hline 罥 & 总 & 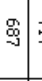 & 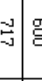 & 官 & ఫ్ల & 棁 & 岕 & : & 吾 & $\vec{g}$ & 僉 & 心్ & 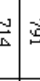 & 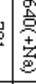 & $\stackrel{9}{\varrho}$ & $\stackrel{9}{\mathrm{~s}}$ & g & 学 & $\mathbb{\Xi}$ & 总 & 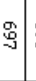 & 8 & ת ת & 3. & & 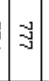 & 缌 & & 颜廷 \\
\hline$\widetilde{\sim}$ & $\approx$ & $\omega$ & $\mathscr{\alpha}$ & 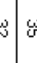 & $\omega$ & 出 & $\mathscr{\omega}$ & $\Leftrightarrow$ & $\Leftrightarrow$ & $\mathrm{A}$ & $\vec{\Delta}$ & $\infty$ & $\omega \approx$ & 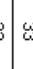 & & 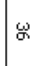 & $\cong$ & 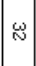 & $\omega$ & $\Rightarrow$ & & & & $\Rightarrow$ & & $\omega$ & $=$ & & 㽞总 \\
\hline 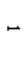 & & $\sigma$. & $\sigma$. & $5 \infty$ & $=$ & $\infty$ & o & $=$ & $\vec{s}$ & $a$ & - & $-I$ & 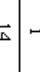 & $\infty$ & $\Delta$ & o & - & $\triangle$ & $=$ & जे & & $=$ & $=$ & $=N$ & & 0. & - & & 思惫 \\
\hline & $\vec{N}$ & & $=$ & & N & $\omega$ & $\omega$ & $\triangle$ & $\sigma$ & л & $\Delta$ & $\omega$. & 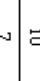 & 5 & $a$ & $\Delta$ & $=$ & $N$ & & $\sim$ & & $N$ & & $n \omega$ & 0 & $N$ & $N$ & & 罾密 \\
\hline & 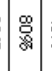 & 黄 & 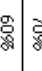 & 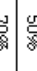 & $\approx$ & 弯 & 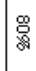 & 急 & 善 & $\stackrel{8}{\circ}$ & 舜 & $\stackrel{8}{8}$ & 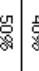 & 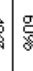 & $\overrightarrow{8}$ & $\omega$ & $\tilde{z}$ & 管 & $\stackrel{2}{\circ}$ & $\stackrel{\circ}{\circ}$ & $\omega$ & 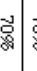 & 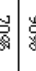 & 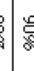 & $\stackrel{8}{\circ}$ & 言 & : & & 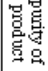 \\
\hline
\end{tabular}

